\title{
Organocatalytic and Highly Stereoselective Direct Vinylogous Mannich
}

\section{Reactions}

Tian-Yu Liu, ${ }^{\dagger}$ Hai-Lei Cui, ${ }^{\dagger}$ Jun Long, ${ }^{\dagger}$ Bang-Jing Li, ${ }^{\ddagger}$ Yong Wu $^{\dagger}{ }^{\dagger}$ Li-Sheng Ding, ${ }^{\ddagger}$ Ying-Chun Chen ${ }^{*},+\S$

\footnotetext{
${ }^{\dagger}$ Key Laboratory of Drug-Targeting of Education Ministry and Department of Medicinal Chemistry, West China School of Pharmacy, Sichuan University, Chengdu 610041,China, ${ }^{\ddagger}$ Chengdu Institute of Biology, Chinese Academy of Sciences, Chengdu, China, ${ }^{\S}$ State Key Laboratory of Biotherapy, West China Hospital, Sichuan University, Chengdu, China
}

E-mail: ycchenhuaxi@yahoo.com.cn

\section{Supporting Information}

Table of Contents

1. General Methods S2

2. General procedure for the direct AVM reaction S2-S7

3. Synthesis of optically active $\delta$-amino acid derivative S8

4. Crystal data and structure refinement for optical 4aa. S9

5. NMR, HRMS and HPLC spectra of the products S10-S70 


\section{General Methods:}

NMR spectra were recorded with tetramethylsilane as the internal standard. Column chromatography was performed using silica gel (200-300 mesh) eluting with ethyl acetate and petroleum ether. Optical rotations were measured at $589 \mathrm{~nm}$ at $20{ }^{\circ} \mathrm{C}$. TLC was performed on glass-backed silica plates. Enantiomeric excess was determined by HPLC analysis on Chiralpak $\mathrm{AS}, \mathrm{AD}$ and OD columns. Commercial grade solvents were dried and purified by standard procedures as specified in Purification of Laboratory Chemicals, 4th Ed (Armarego, W. L. F.; Perrin, D. D. Butterworth Heinemann: 1997).

The bifunctional thiourea-tertiary amine organocatalysts 1a-1g were prepared according to the procedures previously reported. ${ }^{1}$

\section{General procedure for thiourea-tertiary amine catalyzed asymmetric direct vinylogous} Mannich reaction

Catalyst 1 g (0.002 mmol, 2 mol\%), $\alpha, \alpha$-dicyanoolefin 2 (0.1 mmol) and 4A MS (50 mg) were stirred in dry toluene $(0.8 \mathrm{~mL})$ at room temperature. Then $N$-Boc aldimine $3(0.12 \mathrm{mmol})$ in dry toluene $(0.2 \mathrm{~mL})$ were added. After the stated reaction time, the product was purified by flash chromatography on silica gel to give the product 4 . The enantiomeric excess was determined by HPLC analysis on chiral column.

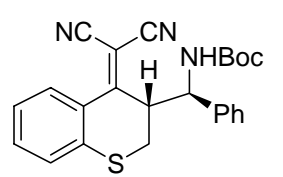

4aa 99\% yield; yellow solid; $\mathrm{R}_{f}=0.1$ (petroleum ether/EtOAc $=20: 1$ ); $[\alpha]_{\mathrm{D}}{ }^{20}=$ $-467.5(\mathrm{c}=0.41$ in $\mathrm{EtOH}) ; 99 \%$ ee, determined by HPLC analysis [Daicel chiralcel OD, $n$-hexane $/ \mathrm{i}$-PrOH $=90 / 10,1.0 \mathrm{~mL} / \mathrm{min}, \lambda=254 \mathrm{~nm}, \mathrm{t}$ (minor) $=$ $7.27 \mathrm{~min}, \mathrm{t}$ (major) =9.08 min]; ${ }^{1} \mathrm{H} \mathrm{NMR}\left(300 \mathrm{MHz}, \mathrm{CDCl}_{3}\right): \delta=7.92(\mathrm{~d}, J=7.6 \mathrm{~Hz}, 1 \mathrm{H})$, 7.47-7.40 (m, 6H), 7.29 (d, $J=7.5 \mathrm{~Hz}, 1 \mathrm{H}), 7.23$ (d, $J=7.6 \mathrm{~Hz}, 1 \mathrm{H}), 4.92-4.85$ (m, 2H), 3.82-3.81 $(\mathrm{m}, 1 \mathrm{H}) ; 3.21(\mathrm{~d}, J=10.3 \mathrm{~Hz}, 1 \mathrm{H}), 2.53(\mathrm{dd}, J=4.3,13.9 \mathrm{~Hz}, 1 \mathrm{H}), 1.37$ (s, 9H) ppm; ${ }^{13} \mathrm{C} \mathrm{NMR}$ $\left(75 \mathrm{MHz}, \mathrm{CDCl}_{3}\right): \delta=172.1,154.3,138.4,137.6,133.6,131.0,129.4,128.8,127.3,126.9,125.1$, 113.4, 113.2, 83.2, 80.3, 55.4, 47.1, 28.5, $28.1 \mathrm{ppm}$; IR (film): $v=3408,2226,1717 \mathrm{~cm}^{-1}$; ESI-HRMS: calcd. for $\mathrm{C}_{24} \mathrm{H}_{23} \mathrm{~N}_{3} \mathrm{O}_{2} \mathrm{~S}+\mathrm{Na} 440.1403$, found 440.1425 .

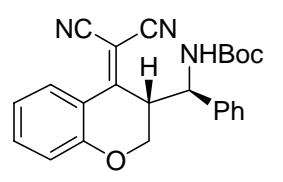

4ba 99\% yield; yellow solid; $\mathrm{R}_{f}=0.1$ (petroleum ether/EtOAc $=20: 1$ ); $[\alpha]_{\mathrm{D}}{ }^{20}=$ -96.7 ( $\mathrm{c}=0.85$ in EtOH); 98\% ee, determined by HPLC analysis [Daicel chiralcel OD, $n$-hexane $/ \mathrm{i}-\mathrm{PrOH}=95 / 5,1.0 \mathrm{~mL} / \mathrm{min}, \lambda=254 \mathrm{~nm}, \mathrm{t}$ (major) $=9.09$ $\min , \mathrm{t}($ minor $)=10.41 \mathrm{~min}] ;{ }^{1} \mathrm{H}$ NMR $\left(300 \mathrm{MHz}, \mathrm{CDCl}_{3}\right): \delta=8.33(\mathrm{~d}, J=8.0 \mathrm{~Hz}, 1 \mathrm{H}), 7.54(\mathrm{t}, J=$ 
$7.8 \mathrm{~Hz}, 1 \mathrm{H}), 7.45-7.34(\mathrm{~m}, 5 \mathrm{H}), 7.11(\mathrm{t}, J=7.7 \mathrm{~Hz}, 1 \mathrm{H}), 7.01$ (d, $J=8.3 \mathrm{~Hz}, 1 \mathrm{H}), 5.04$ (d, $J=9.5$ $\mathrm{Hz}, 1 \mathrm{H}), 4.92$ (t, $J=10.3 \mathrm{~Hz}, 1 \mathrm{H}), 4.13(\mathrm{dd}, J=2.6,12.4 \mathrm{~Hz}, 1 \mathrm{H}), 3.96(\mathrm{dd}, J=1.2,12.4 \mathrm{~Hz}, 1 \mathrm{H})$, $3.34(\mathrm{dd}, J=1.7,14.1 \mathrm{~Hz}, 1 \mathrm{H}), 1.39$ (s, 9H) ppm; ${ }^{13} \mathrm{C} \mathrm{NMR}\left(75 \mathrm{MHz}, \mathrm{CDCl}_{3}\right): \delta=165.8,156.2$, 154.6, 138.2, 136.8, 129.5, 128.8, 128.3, 127.0, 122.0, 118.3, 116.2, 114.1, 113.6, 80.3, 66.3, 55.0, 47.3, 28.3, 28.1 ppm; IR (film): $v=3351,2225,1715 \mathrm{~cm}^{-1}$; ESI-HRMS: calcd. for $\mathrm{C}_{24} \mathrm{H}_{23} \mathrm{~N}_{3} \mathrm{O}_{3}+\mathrm{Na} 424.1632$, found 424.1636.

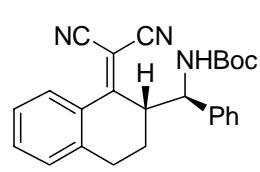

4ca 99\% yield; white solid; $\mathrm{R}_{f}=0.1$ (petroleum ether/EtOAc $=20: 1$ ); $[\alpha]_{\mathrm{D}}^{20}=$ -188.7 (c $=0.46$ in EtOH); 97\% ee, determined by HPLC analysis [Daicel chiralcel OD, $n$-hexane $/ \mathrm{i}-\mathrm{PrOH}=95 / 5,1.0 \mathrm{~mL} / \mathrm{min}, \lambda=254 \mathrm{~nm}, \mathrm{t}$ (minor) $=6.54$ $\min , \mathrm{t}$ (major) $=7.50 \mathrm{~min}] ;{ }^{1} \mathrm{H}$ NMR $\left(300 \mathrm{MHz}, \mathrm{CDCl}_{3}\right): \delta=8.05(\mathrm{~d}, J=7.5 \mathrm{~Hz}, 1 \mathrm{H}), 7.52(\mathrm{t}, J=$ 7.5 Hz, 1H), 7.43-7.35 (m, 4H), 7.33-7.24 (m, 3H), 4.93 (d, J = 9.0 Hz, 1H), 4.53 (t, $J=10.2 \mathrm{~Hz}$, $1 \mathrm{H}), 3.75-3.68(\mathrm{~m}, 1 \mathrm{H}), 2.89-2.84(\mathrm{~m}, 1 \mathrm{H}), 2.72-2.66(\mathrm{~m}, 1 \mathrm{H}), 1.94-1.89(\mathrm{~m}, 1 \mathrm{H}), 1.52-1.47(\mathrm{~m}$, 1H), $1.43(\mathrm{~s}, 9 \mathrm{H}) \mathrm{ppm} ;{ }^{13} \mathrm{C} \mathrm{NMR}\left(75 \mathrm{MHz}, \mathrm{CDCl}_{3}\right): \delta=175.0,154.6,140.0,138.9,133.5,129.8$, $129.4,129.0,128.7,128.6,127.0,114.2,113.6,82.1,80.2,57.4,49.0,28.2,25.8,25.1$ ppm; IR (film): $v=3350,2229,1697 \mathrm{~cm}^{-1}$; ESI-HRMS: calcd. for $\mathrm{C}_{25} \mathrm{H}_{25} \mathrm{~N}_{3} \mathrm{O}_{2}+\mathrm{Na}$ 422.1839, found 422.1857.

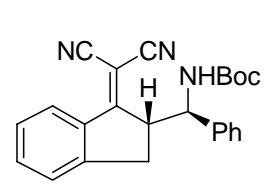

4da $99 \%$ yield; white solid; $\mathrm{R}_{f}=0.1$ (petroleum ether $/$ EtOAc $=20: 1$ ); $[\alpha]_{\mathrm{D}}{ }^{20}=$ -60.5 ( $\mathrm{c}=0.43$ in EtOH); 99\% ee, determined by HPLC analysis [Daicel chiralcel OD, $n$-hexane $/ \mathrm{i}-\mathrm{PrOH}=95 / 5,1.0 \mathrm{~mL} / \mathrm{min}, \lambda=254 \mathrm{~nm}, \mathrm{t}$ (minor) $=8.66$ $\min , \mathrm{t}($ major $)=9.55 \mathrm{~min}] ;{ }^{1} \mathrm{H} \mathrm{NMR}\left(300 \mathrm{MHz}, \mathrm{CDCl}_{3}\right): \delta=8.32(\mathrm{~d}, J=8.0 \mathrm{~Hz}, 1 \mathrm{H}), 7.56(\mathrm{t}, J=$ $7.4 \mathrm{~Hz}, 1 \mathrm{H}), 7.48-7.33(\mathrm{~m}, 7 \mathrm{H}), 5.04(\mathrm{~d}, J=8.9 \mathrm{~Hz}, 1 \mathrm{H}), 4.37$ (t, $J=9.6 \mathrm{~Hz}, 1 \mathrm{H}), 3.92$ (dd, $J=6.2$, $10.0 \mathrm{~Hz}, 1 \mathrm{H}), 3.07(\mathrm{dd}, J=6.2,17.1 \mathrm{~Hz}, 1 \mathrm{H}), 2.71(\mathrm{~d}, J=17.1 \mathrm{~Hz}, 1 \mathrm{H}), 1.46(\mathrm{~s}, 9 \mathrm{H}) \mathrm{ppm} ;{ }^{13} \mathrm{C}$ $\operatorname{NMR}\left(75 \mathrm{MHz}, \mathrm{CDCl}_{3}\right): \delta=178.5,154.9,149.9,139.6,135.6,134.6,129.4,128.6,128.5,127.3$, $126.5,126.0,114.7,112.8,80.4,60.1,54.0,35.1,31.5,28.2$ ppm; IR (film): $v=3438,2221,1705$ $\mathrm{cm}^{-1}$; ESI-HRMS: calcd. for $\mathrm{C}_{24} \mathrm{H}_{23} \mathrm{~N}_{3} \mathrm{O}_{2}+\mathrm{Na} 408.1682$, found 408.1695 .

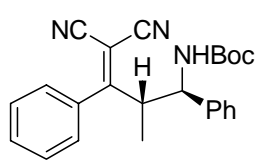

4ea 99\% yield; white solid; $\mathrm{R}_{f}=0.1$ (petroleum ether/EtOAc $=20: 1$ ); $[\alpha]_{\mathrm{D}}{ }^{20}=$ $-10.0(\mathrm{c}=0.37$ in EtOH); 99\% ee, determined by HPLC analysis [Daicel chiralcel AS, $n$-hexane $/ \mathrm{i}$-PrOH $=90 / 10,1.0 \mathrm{~mL} / \mathrm{min}, \lambda=254 \mathrm{~nm}, \mathrm{t}$ (major) $=$ $5.60 \mathrm{~min}, \mathrm{t}($ minor $)=7.01 \mathrm{~min}] ;{ }^{1} \mathrm{H} \mathrm{NMR}\left(300 \mathrm{MHz}, \mathrm{CDCl}_{3}\right): \delta=7.53-7.48(\mathrm{~m}, 3 \mathrm{H}), 7.48-7.40$ (m, 2H), 7.40-7.32 (m, 3H), 7.31-7.20 (m, 2H), $5.02(\mathrm{~d}, J=9.6 \mathrm{~Hz}, 1 \mathrm{H}), 4.67(\mathrm{t}, J=10.5 \mathrm{~Hz}, 1 \mathrm{H})$, 3.59-3.54 (m, 1H), $1.41(\mathrm{~s}, 9 \mathrm{H}), 0.87(\mathrm{~d}, J=6.7 \mathrm{~Hz}, 3 \mathrm{H}) \mathrm{ppm} ;{ }^{13} \mathrm{C} \mathrm{NMR}\left(75 \mathrm{MHz}, \mathrm{CDCl}_{3}\right): \delta=$ 
$183.1,154.6,139.4,133.9,130.7,129.2,129.0,128.3,127.4,127.0,112.6,111.8,89.3,80.5,58.2$, 47.5, 28.2, 16.8 ppm; IR (film): $v=3350,2229,1679 \mathrm{~cm}^{-1}$; ESI-HRMS: calcd. for $\mathrm{C}_{24} \mathrm{H}_{25} \mathrm{~N}_{3} \mathrm{O}_{2}+\mathrm{Na} 410.1839$, found 410.1844 .

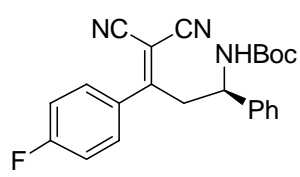

4fa 94\% yield; white solid; $\mathrm{R}_{f}=0.1$ (petroleum ether $/$ EtOAc $=20: 1$ ); $[\alpha]_{\mathrm{D}}{ }^{20}=$ $+54.0(\mathrm{c}=0.52$ in EtOH); 99\% ee, determined by HPLC analysis [Daicel chiralcel AS, $n$-hexane $/ \mathrm{i}-\mathrm{PrOH}=90 / 10,1.0 \mathrm{~mL} / \mathrm{min}, \lambda=254 \mathrm{~nm}, \mathrm{t}$ (major) $=$ $7.44 \mathrm{~min}, \mathrm{t}($ minor $)=9.51 \mathrm{~min}] ;{ }^{1} \mathrm{H} \mathrm{NMR}\left(300 \mathrm{MHz}, \mathrm{CDCl}_{3}\right): \delta=7.55-7.45(\mathrm{~m}, 2 \mathrm{H}), 7.45-7.30$ (m, 3H), 7.25-7.17 (m, 2H), 7.16-7.05 (m, 2H), 4.89 (d, J=7.7 Hz, 1H), $4.58(\mathrm{q}, J=7.4 \mathrm{~Hz}, 1 \mathrm{H})$, 3.74-3.66 (m, 1H), 3.37 (dd, $J=7.0,13.6 \mathrm{~Hz}, 1 \mathrm{H}), 1.45$ (s, 9H) ppm; ${ }^{13} \mathrm{C}$ NMR $\left(75 \mathrm{MHz}, \mathrm{CDCl}_{3}\right)$ : $\delta=174.6,164.8\left(\mathrm{~d},{ }^{1} J_{C, F}=253.5 \mathrm{~Hz}\right), 154.8,138.8,130.5\left(\mathrm{~d},{ }^{3} J_{C, F}=8.9 \mathrm{~Hz}\right), 130.1,129.2,128.7$, 126.4, $116.6\left(\mathrm{~d},{ }^{2} J_{C, F}=22.1 \mathrm{~Hz}\right), 112.7,112.4,86.2,80.3,54.1,44.3,28.3 \mathrm{ppm}$; IR (film): $v=$ 3346, 2236, $1700 \mathrm{~cm}^{-1}$; ESI-HRMS: calcd. for $\mathrm{C}_{23} \mathrm{H}_{22} \mathrm{FN}_{3} \mathrm{O}_{2}+\mathrm{Na} 414.1588$, found 414.1594 .

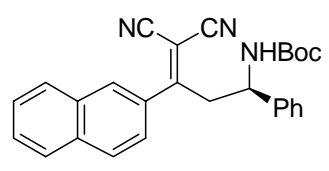

4ga 94\% yield; white solid; $\mathrm{R}_{f}=0.1$ (petroleum ether/EtOAc $=20: 1$ ); $[\alpha]_{\mathrm{D}}{ }^{20}$ $=+45.3(\mathrm{c}=0.54$ in EtOH $) ; 99 \%$ ee, determined by HPLC analysis [Daicel chiralcel AS, $n$-hexane $/ \mathrm{i}-\mathrm{PrOH}=90 / 10,1.0 \mathrm{~mL} / \mathrm{min}, \lambda=254 \mathrm{~nm}$, $\mathrm{t}$ (major) $=$ $8.92 \mathrm{~min}, \mathrm{t}($ minor $)=11.06 \mathrm{~min}] ;{ }^{1} \mathrm{H}$ NMR $\left(300 \mathrm{MHz}, \mathrm{CDCl}_{3}\right): \delta=8.05-7.85(\mathrm{~m}, 4 \mathrm{H}), 7.70-7.50$ $(\mathrm{m}, 3 \mathrm{H}), 7.38-7.30(\mathrm{~m}, 3 \mathrm{H}), 7.18-7.05(\mathrm{~m}, 2 \mathrm{H}), 4.88(\mathrm{~d}, J=7.5 \mathrm{~Hz}, 1 \mathrm{H}), 4.65(\mathrm{~d}, J=7.1 \mathrm{~Hz}, 1 \mathrm{H})$, 3.85-3.78 (m, 1H), $3.50(\mathrm{dd}, J=7.0,13.6 \mathrm{~Hz}, 1 \mathrm{H}), 1.43(\mathrm{~s}, 9 \mathrm{H}) \mathrm{ppm} ;{ }^{13} \mathrm{C}$ NMR $\left(75 \mathrm{MHz}, \mathrm{CDCl}_{3}\right)$ : $\delta=175.9,154.8,139.1,134.8,132.5,131.4,129.2,129.1,128.6,128.6,127.8,127.3,126.5,125.7$, 124.0, 113.0, 112.7, 86.2, 80.2, 54.2, 44.2, 28.2 ppm; IR (film): $v=3365,2225,1676 \mathrm{~cm}^{-1}$; ESI-HRMS: calcd. for $\mathrm{C}_{27} \mathrm{H}_{25} \mathrm{~N}_{3} \mathrm{O}_{2}+\mathrm{Na} 446.1839$, found 446.1828 .

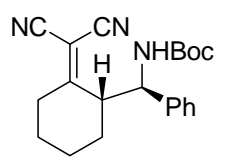

4ha 99\% yield; white solid; $\mathrm{R}_{f}=0.1$ (petroleum ether/EtOAc $=30: 1$ ); $[\alpha]_{\mathrm{D}}{ }^{20}=$ $+42.4(\mathrm{c}=0.59$ in EtOH); 99\% ee, determined by HPLC analysis [Daicel chiralcel $\mathrm{AD}, n$-hexane $/ \mathrm{i}-\mathrm{PrOH}=95 / 5,1.0 \mathrm{~mL} / \mathrm{min}, \lambda=254 \mathrm{~nm}, \mathrm{t}$ (minor) $=4.35 \mathrm{~min}, \mathrm{t}$ $($ major $)=5.14 \mathrm{~min}] ;{ }^{1} \mathrm{H}$ NMR $\left(300 \mathrm{MHz}, \mathrm{CDCl}_{3}\right): \delta=7.45-7.35(\mathrm{~m}, 3 \mathrm{H}), 7.34-7.28(\mathrm{~m}, 2 \mathrm{H}), 5.01$ $(\mathrm{t}, J=9.6 \mathrm{~Hz}, 1 \mathrm{H}), 4.86(\mathrm{~d}, J=9.4 \mathrm{~Hz}, 1 \mathrm{H}), 3.37-3.31(\mathrm{~m}, 1 \mathrm{H}), 3.05-3.01(\mathrm{~m}, 2 \mathrm{H}), 2.11(\mathrm{~d}, J=$ $12.2 \mathrm{~Hz}, 1 \mathrm{H}), 1.79-1.63(\mathrm{~m}, 1 \mathrm{H}), 1.59-1.36(\mathrm{~m}, 4 \mathrm{H}), 1.41(\mathrm{~s}, 9 \mathrm{H}) \mathrm{ppm} ;{ }^{13} \mathrm{C} \mathrm{NMR}(75 \mathrm{MHz}$, $\left.\mathrm{CDCl}_{3}\right): \delta=186.1,155.1,138.9,129.4,128.6,126.9,112.4,111.5,85.1,80.5,56.0,49.6,31.8$, 28.9, 28.2, 27.3, 19.7 ppm; IR (film): $v=3357,2235,1696 \mathrm{~cm}^{-1}$; ESI-HRMS: calcd. for $\mathrm{C}_{21} \mathrm{H}_{25} \mathrm{~N}_{3} \mathrm{O}_{2}+\mathrm{Na} 374.1839$, found 374.1847. 


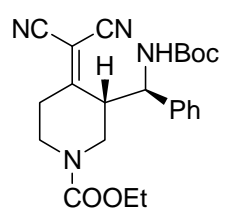

4ia 99\% yield; white solid; $\mathrm{R}_{f}=0.1$ (petroleum ether/EtOAc $=9: 1$ ); $[\alpha]_{\mathrm{D}}{ }^{20}=$ $+120.3(\mathrm{c}=0.68$ in EtOH); 98\% ee, determined by HPLC analysis [Daicel chiralcel OD, $n$-hexane $/ \mathrm{i}-\mathrm{PrOH}=95 / 5,1.0 \mathrm{~mL} / \mathrm{min}, \lambda=254 \mathrm{~nm}, \mathrm{t}$ (minor) $=10.40 \mathrm{~min}, \mathrm{t}$ (major) $=12.05 \mathrm{~min}] ;{ }^{1} \mathrm{H} \mathrm{NMR}\left(300 \mathrm{MHz}, \mathrm{CDCl}_{3}\right): \delta=7.46-7.33(\mathrm{~m}, 5 \mathrm{H}), 4.86(\mathrm{~s}, 2 \mathrm{H}), 4.49$ (br.s, 1H), 4.25-4.05 (m, 2H), 3.93 (br.s, 1H), 3.42-3.30 (m, 2H), 2.98 (d, J=15.6 Hz, 1H), 2.87-2.75 $(\mathrm{m}, 2 \mathrm{H}), 1.41(\mathrm{~s}, 9 \mathrm{H}), 1.25(\mathrm{t}, J=7.1 \mathrm{~Hz}, 3 \mathrm{H}) \mathrm{ppm} ;{ }^{13} \mathrm{C} \mathrm{NMR}\left(75 \mathrm{MHz}, \mathrm{CDCl}_{3}\right): \delta=180.6,155.0$, $138.4,129.4,128.9,127.4,112.0,110.9,87.1,80.7,62.0,56.1,50.2,44.4,43.2,31.7,28.1,14.4$ ppm; IR (film): $v=3307,2232,1678 \mathrm{~cm}^{-1}$; ESI-HRMS: calcd. for $\mathrm{C}_{23} \mathrm{H}_{28} \mathrm{~N}_{4} \mathrm{O}_{4}+\mathrm{Na} 447.2003$, found 447.2010 .

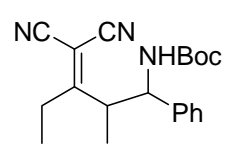

4ja (major) 76\% yield; colorless oil; $\mathrm{R}_{f}=0.1$ (petroleum ether/EtOAc $=25: 1$ ); $[\alpha]_{\mathrm{D}}{ }^{20}=+39.0(\mathrm{c}=0.50$ in EtOH $) ; 98 \%$ ee, determined by HPLC analysis [Daicel chiralcel AS, $n$-hexane $/ \mathrm{i}-\mathrm{PrOH}=95 / 5,1.0 \mathrm{~mL} / \mathrm{min}, \lambda=254 \mathrm{~nm}, \mathrm{t}$ (major) $=5.27 \mathrm{~min}, \mathrm{t}$ (minor) $=$ $6.06 \mathrm{~min}$ ]; ${ }^{1} \mathrm{H}$ NMR (300 MHz, $\left.\mathrm{CDCl}_{3}\right): \delta=7.43-7.33$ (m, 3H), 7.33-7.28 (m, 2H), 4.86 (d, $J=8.9$ $\mathrm{Hz}, 1 \mathrm{H}), 4.57(\mathrm{t}, J=10.1 \mathrm{~Hz}, 1 \mathrm{H}), 3.41-3.35(\mathrm{~m}, 1 \mathrm{H}), 2.79-2.63(\mathrm{~m}, 2 \mathrm{H}), 1.40(\mathrm{~s}, 9 \mathrm{H}), 1.29$ (t, $J=$ $7.7 \mathrm{~Hz}, 3 \mathrm{H}), 0.93(\mathrm{~d}, J=6.7 \mathrm{~Hz}, 3 \mathrm{H}) \mathrm{ppm} ;{ }^{13} \mathrm{C} \mathrm{NMR}\left(75 \mathrm{MHz}, \mathrm{CDCl}_{3}\right): \delta=189.5,154.8,138.9$, 129.3, 128.6, 127.1, 112.5, 111.7, 87.7, 80.5, 58.6, 48.4, 28.2, 26.3, 15.2, 13.7 ppm; IR (film): $v=$ 3363, 2232, $1702 \mathrm{~cm}^{-1}$; ESI-HRMS: calcd. for $\mathrm{C}_{20} \mathrm{H}_{25} \mathrm{~N}_{3} \mathrm{O}_{2}+\mathrm{Na} 362.1839$, found 362.1842 .

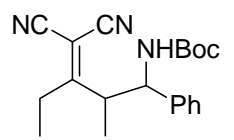

4ja (minor) 17\% yield; colorless oil; $\mathrm{R}_{f}=0.1$ (petroleum ether/EtOAc $=20: 1$ ); $[\alpha]_{\mathrm{D}}{ }^{20}=+37.8(\mathrm{c}=0.53$ in $\mathrm{EtOH}) ; 78 \%$ ee, determined by HPLC analysis [Daicel chiralcel AD, $n$-hexane $/ \mathrm{i}-\mathrm{PrOH}=97 / 3,1.0 \mathrm{~mL} / \mathrm{min}, \lambda=254 \mathrm{~nm}, \mathrm{t}$ (minor) $=18.91 \mathrm{~min}, \mathrm{t}$ (major) $=20.42 \mathrm{~min}] ;{ }^{1} \mathrm{H}$ NMR $\left(300 \mathrm{MHz}, \mathrm{CDCl}_{3}\right): \delta=7.36-7.31(\mathrm{~m}, 3 \mathrm{H}), 7.21-7.18(\mathrm{~m}, 2 \mathrm{H}), 4.93(\mathrm{~d}, J=$ $8.0 \mathrm{~Hz}, 1 \mathrm{H}), 4.81(\mathrm{t}, J=9.5 \mathrm{~Hz}, 1 \mathrm{H}), 3.48-3.44(\mathrm{~m}, 1 \mathrm{H}), 2.48-2.36(\mathrm{~m}, 2 \mathrm{H}), 1.43(\mathrm{~s}, 9 \mathrm{H}), 1.36(\mathrm{~d}$, $J=6.8 \mathrm{~Hz}, 3 \mathrm{H}), 1.17(\mathrm{t}, J=7.7 \mathrm{~Hz}, 3 \mathrm{H}) \mathrm{ppm} ;{ }^{13} \mathrm{C} \mathrm{NMR}\left(75 \mathrm{MHz}, \mathrm{CDCl}_{3}\right): \delta=188.2,155.2$, 139.1, 129.2, 128.6, 126.4, 111.8, 111.4, 87.2, 80.4, 57.7, 48.4, 28.3, 25.8, 15.5, 13.7 ppm; IR (film): $v=3375,2229,1697 \mathrm{~cm}^{-1}$; ESI-HRMS: calcd. for $\mathrm{C}_{20} \mathrm{H}_{25} \mathrm{~N}_{3} \mathrm{O}_{2}+\mathrm{Na}$ 362.1839, found 362.1856.

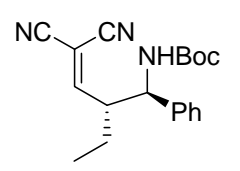

4ka 99\% yield; white solid; $\mathrm{R}_{f}=0.1$ (petroleum ether/EtOAc $=35: 1$ ); $[\alpha]_{\mathrm{D}}{ }^{20}=+6.7$ $(\mathrm{c}=0.60$ in $\mathrm{EtOH}) ; 99 \%$ ee, determined by HPLC analysis [Daicel chiralcel AD, $n$-hexane $/ \mathrm{i}-\mathrm{PrOH}=90 / 10,1.0 \mathrm{~mL} / \mathrm{min}, \lambda=254 \mathrm{~nm}, \mathrm{t}$ (minor) $=4.90 \mathrm{~min}, \mathrm{t}$ (major) $=5.26 \mathrm{~min}] ;{ }^{1} \mathrm{H}$ NMR $\left(300 \mathrm{MHz}, \mathrm{CDCl}_{3}\right): \delta=7.45-7.39(\mathrm{~m}, 5 \mathrm{H}), 6.21(\mathrm{dt}, J=6.2,15.3 \mathrm{~Hz}, 1 \mathrm{H})$, $5.45(\mathrm{~d}, J=10.2 \mathrm{~Hz}, 1 \mathrm{H}), 5.39(\mathrm{~d}, J=15.3 \mathrm{~Hz}, 1 \mathrm{H}), 5.26(\mathrm{~d}, J=10.2 \mathrm{~Hz}, 1 \mathrm{H}), 2.17-2.10(\mathrm{~m}, 2 \mathrm{H})$, 
$1.44(\mathrm{~s}, 9 \mathrm{H}), 1.01(\mathrm{t}, J=7.4 \mathrm{~Hz}, 3 \mathrm{H}) \mathrm{ppm} ;{ }^{13} \mathrm{C} \mathrm{NMR}\left(75 \mathrm{MHz}, \mathrm{CDCl}_{3}\right): \delta=154.3,140.9,134.0$, 129.7, 128.9, 127.7, 118.2, 113.2, 81.3, 73.9, 60.0, 28.1, 25.0, 12.4 ppm; IR (film): $v=3348,3313$, 2251, $1700 \mathrm{~cm}^{-1}$; ESI-HRMS: calcd. for $\mathrm{C}_{19} \mathrm{H}_{23} \mathrm{~N}_{3} \mathrm{O}_{2}+\mathrm{Na} 348.1682$, found 348.1687.

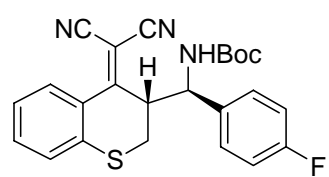

4ab 99\% yield; yellow solid; $\mathrm{R}_{f}=0.1$ (petroleum ether/EtOAc $=20: 1$ ); $[\alpha]_{\mathrm{D}}{ }^{20}$ $=-386.0(\mathrm{c}=0.60$ in EtOH $) ;>99 \%$ ee, determined by HPLC analysis [Daicel chiralcel OD, $n$-hexane $/ \mathrm{i}-\mathrm{PrOH}=90 / 10,1.0 \mathrm{~mL} / \mathrm{min}, \lambda=254 \mathrm{~nm}, \mathrm{t}$ (minor) $=$ $6.54 \mathrm{~min}, \mathrm{t}($ major $)=9.99 \mathrm{~min}] ;{ }^{1} \mathrm{H} \operatorname{NMR}\left(300 \mathrm{MHz}, \mathrm{CDCl}_{3}\right): \delta=7.90(\mathrm{~d}, J=7.9 \mathrm{~Hz}, 1 \mathrm{H}), 7.45(\mathrm{t}$, $J=8.0 \mathrm{~Hz}, 1 \mathrm{H}), 7.40-7.35$ (m, 2H), 7.29 (d, $J=7.9 \mathrm{~Hz}, 1 \mathrm{H}), 7.22$ (d, $J=7.4 \mathrm{~Hz}, 1 \mathrm{H}), 7.09$ (t, $J=$ $8.5 \mathrm{~Hz}, 1 \mathrm{H}), 4.91-4.85(\mathrm{~m}, 2 \mathrm{H}), 3.77-3.74(\mathrm{~m}, 1 \mathrm{H}), 3.25(\mathrm{dd}, J=2.2,11.0 \mathrm{~Hz}, 1 \mathrm{H}), 2.51(\mathrm{dd}, J=$ 4.0, $9.9 \mathrm{~Hz}, 1 \mathrm{H}), 1.36(\mathrm{~s}, 9 \mathrm{H}) \mathrm{ppm} ;{ }^{13} \mathrm{C} \mathrm{NMR}\left(75 \mathrm{MHz}, \mathrm{CDCl}_{3}\right): \delta=171.9,162.7\left(\mathrm{~d},{ }^{1} J_{C, F}=246.8\right.$ $\mathrm{Hz}), 154.2,137.4,134.4,133.8,130.9,128.7\left(\mathrm{~d},{ }^{3} J_{C, F}=8.2 \mathrm{~Hz}\right), 127.3,125.2,116.3\left(\mathrm{~d},{ }^{2} J_{C, F}=\right.$ $21.5 \mathrm{~Hz}$ ), 115.3, 113.3, 113.1, 83.2, 80.5, 54.6, 46.7, 28.5, 28.2 ppm; IR (film): $v=3408,2226$, $1709 \mathrm{~cm}^{-1}$; ESI-HRMS: calcd. for $\mathrm{C}_{24} \mathrm{H}_{22} \mathrm{FN}_{3} \mathrm{O}_{2} \mathrm{~S}+\mathrm{Na} 458.1309$, found 458.1304 .

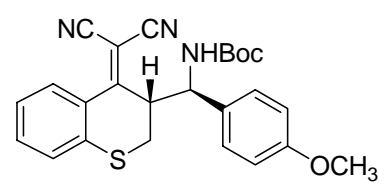

4ac 99\% yield; yellow solid; $\mathrm{R}_{f}=0.1$ (petroleum ether/EtOAc $=20: 1$ ); $[\alpha]_{\mathrm{D}}{ }^{20}=-203.8(\mathrm{c}=0.73$ in EtOH $) ; 99 \%$ ee, determined by HPLC analysis [Daicel chiralcel OD, $n$-hexane $/ \mathrm{i}-\mathrm{PrOH}=90 / 10,1.0 \mathrm{~mL} / \mathrm{min}, \lambda=254 \mathrm{~nm}$, $\mathrm{t}($ minor $)=8.77 \mathrm{~min}, \mathrm{t}$ (major) $=12.25 \mathrm{~min}] ;{ }^{1} \mathrm{H} \mathrm{NMR}\left(300 \mathrm{MHz}, \mathrm{CDCl}_{3}\right): \delta=7.90(\mathrm{~d}, J=7.5 \mathrm{~Hz}$, $1 \mathrm{H}), 7.44$ (t, $J=7.6 \mathrm{~Hz}, 1 \mathrm{H}), 7.32-7.20(\mathrm{~m}, 2 \mathrm{H}), 6.92$ (d, $J=8.7 \mathrm{~Hz}, 2 \mathrm{H}), 4.91-4.79$ (m, 2H), 3.81 (s, 3H), 3.78-3.75 (m, 1H), 3.21 (d, $J=13.2 \mathrm{~Hz}, 1 \mathrm{H}), 2.53$ (dd, $J=4.0,13.6 \mathrm{~Hz}, 1 \mathrm{H}), 1.37$ (s, 9H) ppm; ${ }^{13} \mathrm{C}$ NMR $\left(75 \mathrm{MHz}, \mathrm{CDCl}_{3}\right): \delta=172.3,159.8,154.3,137.6,133.6,131.0,130.5,128.1$, 127.3, 126.8, 125.1, 114.7, 113.4, 113.2, 83.1, 80.2, 55.3, 54.9, 47.3, 28.6, 28.2 ppm; IR (film): $v=$ 3376, 2226, $1711 \mathrm{~cm}^{-1}$; ESI-HRMS: calcd. for $\mathrm{C}_{25} \mathrm{H}_{25} \mathrm{~N}_{3} \mathrm{O}_{3} \mathrm{~S}+\mathrm{Na} 470.1509$, found 470.1516.

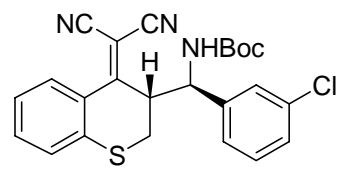

4ad 99\% yield; yellow solid; $\mathrm{R}_{f}=0.1$ (petroleum ether/EtOAc $=20: 1$ ); $[\alpha]_{\mathrm{D}}{ }^{20}=-350.0(\mathrm{c}=0.70$ in EtOH $) ; 98 \%$ ee, determined by HPLC analysis [Daicel chiralcel AD, $n$-hexane $/ \mathrm{i}-\mathrm{PrOH}=90 / 10,2.0 \mathrm{~mL} / \mathrm{min}, \lambda=254 \mathrm{~nm}, \mathrm{t}$ $($ minor $)=2.44 \mathrm{~min}, \mathrm{t}$ (major) $=3.75 \mathrm{~min}] ;{ }^{1} \mathrm{H} \mathrm{NMR}\left(300 \mathrm{MHz}, \mathrm{CDCl}_{3}\right): \delta=7.89(\mathrm{~d}, J=7.6 \mathrm{~Hz}$, $1 \mathrm{H}), 7.45$ (t, $J=7.2 \mathrm{~Hz}, 1 \mathrm{H}), 7.39$ (s, 1H), 7.35-7.28 (m, 4H), 7.22 (d, $J=7.2 \mathrm{~Hz}, 1 \mathrm{H}), 4.99$ (d, $J=$ $8.2 \mathrm{~Hz}, 1 \mathrm{H}), 4.87(\mathrm{t}, J=10.2 \mathrm{~Hz}, 1 \mathrm{H}), 3.77-3.74(\mathrm{~m}, 1 \mathrm{H}), 3.27(\mathrm{~d}, J=13.7 \mathrm{~Hz}, 1 \mathrm{H}), 2.54(\mathrm{dd}, J=$ 4.0, $14.0 \mathrm{~Hz}, 1 \mathrm{H}), 1.36$ (s, 9H) ppm; ${ }^{13} \mathrm{C} \mathrm{NMR}\left(75 \mathrm{MHz}, \mathrm{CDCl}_{3}\right): \delta=171.7,154.2,140.5,137.4$, 135.2, 133.8, 130.8, 130.6, 129.0, 127.2, 126.9, 126.2, 125.1, 125.0, 113.2, 113.0, 80.5, 68.3, 54.7, 46.3, 28.8, 28.4 ppm; IR (film): $v=3426,2228,1688 \mathrm{~cm}^{-1}$; ESI-HRMS: calcd. for 


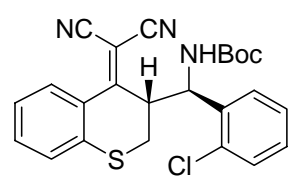

4ae 99\% yield; yellow solid; $\mathrm{R}_{f}=0.1$ (petroleum ether/EtOAc $=20: 1$ ); $[\alpha]_{\mathrm{D}}{ }^{20}=$ $-351.6(\mathrm{c}=0.73$ in EtOH); $>99.5 \%$ ee, determined by HPLC analysis [Daicel chiralcel AD, $n$-hexane $/ \mathrm{i}-\mathrm{PrOH}=90 / 10,2.0 \mathrm{~mL} / \mathrm{min}, \lambda=254 \mathrm{~nm}$, $\mathrm{t}$ (major) $=$ $6.40 \mathrm{~min}] ;{ }^{1} \mathrm{H}$ NMR $\left(300 \mathrm{MHz}, \mathrm{CDCl}_{3}\right): \delta=7.86(\mathrm{~d}, J=7.8 \mathrm{~Hz}, 1 \mathrm{H}), 7.47-7.41(\mathrm{~m}, 3 \mathrm{H})$, 7.34-7.28 (m, 3H), 7.25-7.20 (m, 1H), 5.59 (br.s, 1H), 5.19 (t, $J=8.4$ Hz, 1H), 4.38 (br.s, 1H), $3.28(\mathrm{~d}, J=13.3 \mathrm{~Hz}, 1 \mathrm{H}), 2.54(\mathrm{dd}, J=3.8,13.9 \mathrm{~Hz}, 1 \mathrm{H}), 1.36(\mathrm{~s}, 9 \mathrm{H}) \mathrm{ppm} ;{ }^{13} \mathrm{C} \mathrm{NMR}(75 \mathrm{MHz}$, $\left.\mathrm{CDCl}_{3}\right): \delta=172.0,154.3,137.2,134.9,133.6,132.8,131.0,130.6,129.6,127.5,127.2$, 126.4, 125.2, 113.2, 83.6, 80.4, 68.5, 54.8, 46.3, 28.5, 28.2 ppm; IR (film): $v=3354,2225,1708 \mathrm{~cm}^{-1}$; ESI-HRMS: calcd. for $\mathrm{C}_{24} \mathrm{H}_{22} \mathrm{ClN}_{3} \mathrm{O}_{2} \mathrm{~S}+\mathrm{Na} 474.1013$, found 474.0996.

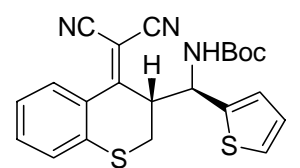

4af 99\% yield; yellow solid; $\mathrm{R}_{f}=0.1$ (petroleum ether/EtOAc $=20: 1$ ); $[\alpha]_{\mathrm{D}}{ }^{20}=$ -314.0 ( $\mathrm{c}=0.70$ in EtOH); 98\% ee, determined by HPLC analysis [Daicel chiralcel AD, $n$-hexane $/ \mathrm{i}-\mathrm{PrOH}=90 / 10,1.0 \mathrm{~mL} / \mathrm{min}, \lambda=254 \mathrm{~nm}, \mathrm{t}$ (minor) $=$ $6.51 \mathrm{~min}, \mathrm{t}$ (major) $=12.10 \mathrm{~min}] ;{ }^{1} \mathrm{H}$ NMR $\left(300 \mathrm{MHz}, \mathrm{CDCl}_{3}\right): \delta=7.86(\mathrm{~d}, J=7.7 \mathrm{~Hz}, 1 \mathrm{H}), 7.44$ $(\mathrm{t}, J=7.6 \mathrm{~Hz}, 1 \mathrm{H}), 7.32-7.29(\mathrm{~m}, 2 \mathrm{H}), 7.24-7.19(\mathrm{~m}, 1 \mathrm{H}), 7.13(\mathrm{~d}, J=2.7 \mathrm{~Hz}, 1 \mathrm{H}), 7.01(\mathrm{dd}, J=$ 3.5, $5.1 \mathrm{~Hz}, 1 \mathrm{H}), 5.23(\mathrm{t}, J=10.3 \mathrm{~Hz}, 1 \mathrm{H}), 4.85$ (d, $J=8.1 \mathrm{~Hz}, 1 \mathrm{H}), 3.84-3.78(\mathrm{~m}, 1 \mathrm{H}), 3.33(\mathrm{~d}, J$ $=11.1 \mathrm{~Hz}, 1 \mathrm{H}), 2.75(\mathrm{~d}, J=10.8 \mathrm{~Hz}, 1 \mathrm{H}), 1.37(\mathrm{~s}, 9 \mathrm{H}) \mathrm{ppm} ;{ }^{13} \mathrm{C} \mathrm{NMR}\left(75 \mathrm{MHz}, \mathrm{CDCl}_{3}\right): \delta=$ 171.6, 154.1, 141.3, 137.5, 133.7, 130.9, 127.3, 127.2, 126.3, 125.7, 125.1, 113.1, 83.4, 80.6, 50.7, 47.3, 28.8, 28.1 ppm; IR (film): $v=3380,2229,1708 \mathrm{~cm}^{-1}$; ESI-HRMS: calcd. for $\mathrm{C}_{22} \mathrm{H}_{21} \mathrm{~N}_{3} \mathrm{O}_{2} \mathrm{~S}_{2}+\mathrm{Na} 446.0967$, found 446.0969.

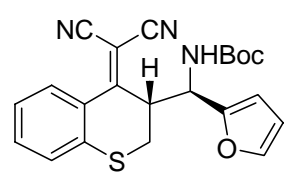

4ag 99\% yield; yellow solid; $\mathrm{R}_{f}=0.1$ (petroleum ether/EtOAc $=20: 1$ ); $[\alpha]_{\mathrm{D}}{ }^{20}=$ -295.1 ( $c=0.41$ in EtOH); 96\% ee, determined by HPLC analysis [Daicel chiralcel AD, $n$-hexane $/ \mathrm{i}-\mathrm{PrOH}=90 / 10,1.0 \mathrm{~mL} / \mathrm{min}, \lambda=254 \mathrm{~nm}, \mathrm{t}$ (minor) $=$ $5.94 \mathrm{~min}, \mathrm{t}($ major $)=8.04 \mathrm{~min}] ;{ }^{1} \mathrm{H} \operatorname{NMR}\left(300 \mathrm{MHz}, \mathrm{CDCl}_{3}\right): \delta=7.86(\mathrm{~d}, J=8.0 \mathrm{~Hz}, 1 \mathrm{H}), 7.43(\mathrm{t}$, $J=8.5 \mathrm{~Hz}, 1 \mathrm{H}), 7.27-7.19(\mathrm{~m}, 2 \mathrm{H}), 6.37-6.34(\mathrm{~m}, 2 \mathrm{H}), 5.06(\mathrm{~d}, J=5.4 \mathrm{~Hz}, 1 \mathrm{H}), 3.95-3.90(\mathrm{~m}, 1 \mathrm{H})$, $3.29(\mathrm{dd}, J=3.9,13.7 \mathrm{~Hz}, 1 \mathrm{H}), 2.61(\mathrm{dd}, J=3.8,13.4 \mathrm{~Hz}, 1 \mathrm{H}), 1.38(\mathrm{~s}, 9 \mathrm{H}) \mathrm{ppm} ;{ }^{13} \mathrm{C}$ NMR $(75$ $\left.\mathrm{MHz}, \mathrm{CDCl}_{3}\right): \delta=171.3,154.2,150.1,143.0,137.4,133.7,131.0,127.2,125.1,113.2,110.5$, 109.0, 80.4, 48.9, 44.7, 28.4, 28.1 ppm; IR (film): $v=3392,2229,1715 \mathrm{~cm}^{-1}$; ESI-HRMS: calcd. for $\mathrm{C}_{22} \mathrm{H}_{21} \mathrm{~N}_{3} \mathrm{O}_{3} \mathrm{~S}+\mathrm{Na} 430.1196$, found 430.1193 .

\section{Synthesis of optically active $\delta$-amino acid derivative}




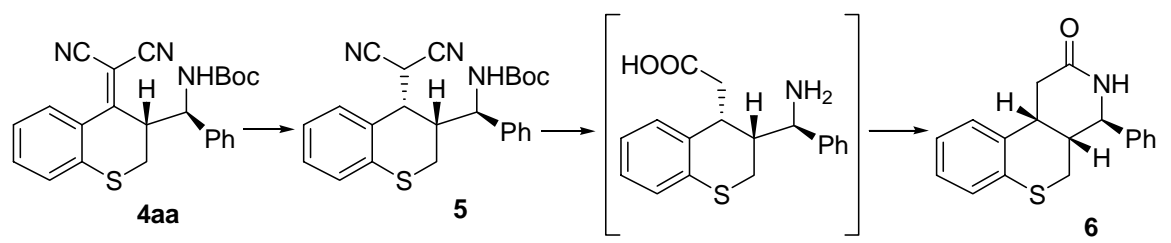

Hantzsch ester $(291 \mathrm{mg}, 1.15 \mathrm{mmol})$ was added to a stirred solution of direct vinylogous Mannich product 4aa $(96 \mathrm{mg}, 0.23 \mathrm{mmol})$ in $\mathrm{DCM} / \mathrm{EtOH}(1: 1,10.0 \mathrm{~mL})$. The solution was stirred at $50{ }^{\circ} \mathrm{C}$ and monitored by TLC. After $48 \mathrm{~h}$, the mixture was concentrated and purified by flash chromatography on silica gel to give the reduced product $5.92 \%$ yield $(89 \mathrm{mg})$; white solid; $\mathrm{R}_{f}=$ 0.1 (petroleum ether/EtOAc $=10: 1) ;[\alpha]_{\mathrm{D}}{ }^{20}=+185.4(\mathrm{c}=1.06$ in $\mathrm{EtOH}) ; 99 \%$ ee, determined by HPLC analysis [Daicel chiralcel OD, $n$-hexane $/ \mathrm{i}-\mathrm{PrOH}=90 / 10,1.0 \mathrm{~mL} / \mathrm{min}, \lambda=254 \mathrm{~nm}, \mathrm{t}$ (major) $=9.06$ min, $\mathrm{t}($ minor $)=18.38 \mathrm{~min}] ;{ }^{1} \mathrm{H}$ NMR $\left(300 \mathrm{MHz}, \mathrm{CDCl}_{3}\right): \delta=7.43-7.36(\mathrm{~m}, 4 \mathrm{H}), 7.28-7.23$ $(\mathrm{m}, 3 \mathrm{H}), 7.19(\mathrm{~d}, J=6.8 \mathrm{~Hz}, 1 \mathrm{H}), 7.16-7.11(\mathrm{~m}, 1 \mathrm{H}), 4.98(\mathrm{~s}, 1 \mathrm{H}), 4.88(\mathrm{~d}, J=8.6 \mathrm{~Hz}, 1 \mathrm{H}), 4.66(\mathrm{t}$, $J=10.8 \mathrm{~Hz}, 1 \mathrm{H}), 3.68(\mathrm{~s}, 1 \mathrm{H}), 3.07(\mathrm{t}, J=12.8 \mathrm{~Hz}, 1 \mathrm{H}), 2.63-2.47(\mathrm{~m}, 2 \mathrm{H}), 1.49(\mathrm{~s}, 9 \mathrm{H}) \mathrm{ppm} ;{ }^{13} \mathrm{C}$ $\operatorname{NMR}\left(75 \mathrm{MHz}, \mathrm{CDCl}_{3}\right): \delta=155.8,138.9,133.5,131.2,129.7,129.5,128.9,128.8,126.8,126.5$, 124.5, 113.3, 112.0, 81.5, 56.9, 43.3, 41.4, 28.3, 25.1, 24.7 ppm; IR (film): $v=3213,2240,1665$ $\mathrm{cm}^{-1}$; ESI-HRMS: calcd. for $\mathrm{C}_{24} \mathrm{H}_{25} \mathrm{~N}_{3} \mathrm{O}_{2} \mathrm{~S}+\mathrm{Na} 442.1560$, found 442.1543 .

To a solution of concd. $\mathrm{HCl}(5.0 \mathrm{~mL})$ was added hydrogenated product $5(68.0 \mathrm{mg}, 0.162 \mathrm{mmol})$. The mixture was stirred at $90{ }^{\circ} \mathrm{C}$ for 4 hours and a homogeneous solution was obtained. Then excess $\mathrm{HCl}$ solution was removed under vacuum, and the residue was diluted with water $(5 \mathrm{~mL})$. After adjusting the $\mathrm{pH}$ of the solution is about 10 by $\mathrm{K}_{2} \mathrm{CO}_{3}$, (Boc) ${ }_{2} \mathrm{O}(38.9 \mathrm{mg}, 0.179 \mathrm{mmol})$ in EtOH $(3.0 \mathrm{~mL})$ was added. The mixture was stirred at room temperature overnight. The mixture was extracted with EtOAc $(3 \times 5 \mathrm{~mL})$. The combined organic layers were washed with water and brine, dried over $\mathrm{Na}_{2} \mathrm{SO}_{4}$ and concentrated. The residue was purified by flash chromatography on silica gel to give the cyclic product 6. $91 \%$ yield for two steps $(44 \mathrm{mg}) ; \mathrm{R}_{f}=0.1$ (petroleum ether/EtOAc $=3: 1) ;[\alpha]_{\mathrm{D}}{ }^{20}=-5.3(\mathrm{c}=0.36 \mathrm{in} \mathrm{EtOH}) ; 99 \%$ ee, determined by HPLC analysis [Daicel chiralcel AD, $n$-hexane $/ \mathrm{i}-\mathrm{PrOH}=90 / 10,1.0 \mathrm{~mL} / \mathrm{min}, \lambda=254 \mathrm{~nm}, \mathrm{t}$ (minor) $=18.24 \mathrm{~min}, \mathrm{t}$ (major) $=22.12 \mathrm{~min}] ;{ }^{1} \mathrm{H}$ NMR $\left(300 \mathrm{MHz}, \mathrm{CDCl}_{3}\right): \delta=7.43-7.30(\mathrm{~m}, 5 \mathrm{H}), 7.17-7.09(\mathrm{~m}, 2 \mathrm{H})$, 7.09-6.99 (m, 2H), $6.08(\mathrm{~s}, 1 \mathrm{H}), 4.58(\mathrm{dd}, J=2.6 \mathrm{~Hz}, 5.0 \mathrm{~Hz}, 1 \mathrm{H}), 3.27-3.20(\mathrm{~m}, 1 \mathrm{H}), 3.13-3.08$ $(\mathrm{m}, 2 \mathrm{H}), 2.86-2.70(\mathrm{~m}, 2 \mathrm{H}), 2.60-2.52(\mathrm{~m}, 1 \mathrm{H}) \mathrm{ppm} ;{ }^{13} \mathrm{C} \mathrm{NMR}\left(75 \mathrm{MHz}, \mathrm{CDCl}_{3}\right): \delta=170.6,141.1$, 134.0, 131.7, 128.9, 128.8, 128.2, 127.3, 126.6, 126.5, 124.7, 59.6, 38.6, 35.4, 32.7, 26.9 ppm; IR (film): $v=3389,1695 \mathrm{~cm}^{-1}$; ESI-HRMS: calcd. for $\mathrm{C}_{18} \mathrm{H}_{17} \mathrm{NOS}+\mathrm{Na} 318.0923$, found 318.0927 .

\section{References:}


(1) (a) Li, B.-J.; Jiang, L.; Liu, M.; Chen, Y.-C.; Ding, L.-S.; Wu, Y. Synlett 2005, 603. (b) Liu, T.-Y.; Long, J.; Li, B.-J.; Jiang, L.; Li, R.; Wu, Y.; Ding, L.-S.; Chen, Y.-C.; Org. Biomol. Chem. 2006, 4, 2097. (c) Okino, T.; Hoashi, Y.; Takemoto, Y.; J. Am. Chem. Soc. 2003, 125, 12672. (d) Berkessel, A.; Cleemann, F.; Mukherjee, S. Angew. Chem., Int. Ed. 2005, 44, 7466. (e) McCooey, S. H.; Connon, S. J. Angew. Chem., Int. Ed. 2005, $44,6367$.

\section{Crystal data and structure refinement for opticalpure 4aa}

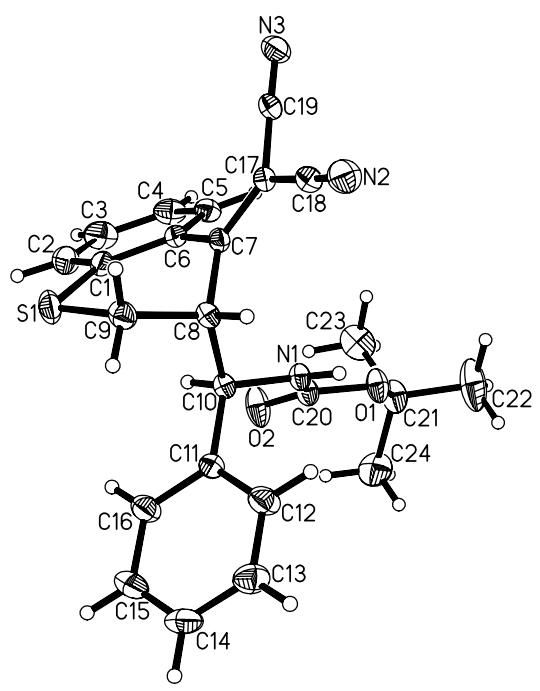

Crystal size

Theta range for data collection

Limiting indices

Reflections collected / unique

Completeness to theta $=25.99$

Absorption correction

Max. and min. transmission

Refinement method

Data / restraints / parameters

Goodness-of-fit on $\mathrm{F}^{\wedge} 2$

Final $\mathrm{R}$ indices [I $>2 \operatorname{sigma}(\mathrm{I})]$

$\mathrm{R}$ indices (all data)

Absolute structure parameter

Extinction coefficient

Largest diff. peak and hole
Identification code $\quad$ 4aa

Empirical formula

C24 H23 N3 O2 S

Formula weight

417.51

Temperature

289(2) K

Wavelength

$0.71073 \mathrm{~A}$

Crystal system, space group Monoclinic, P2(1)

Unit cell dimensions $\mathrm{a}=7.313(1) \mathrm{A} \quad \mathrm{alpha}=90 \mathrm{deg}$.

$$
\begin{array}{ll}
\mathrm{b}=12.477(1) \mathrm{A} & \text { beta }=91.17(1) \mathrm{deg} . \\
\mathrm{c}=12.264(1) \mathrm{A} & \text { gamma }=90 \mathrm{deg} .
\end{array}
$$

Volume

$1118.8(2) \mathrm{A}^{\wedge} 3$

Z, Calculated density

2, $\quad 1.239 \mathrm{Mg} / \mathrm{m}^{\wedge} 3$

Absorption coefficient

$0.169 \mathrm{~mm}^{\wedge}-1$

$\mathrm{F}(000)$

440

$0.56 \times 0.46 \times 0.30 \mathrm{~mm}$

1.66 to $25.99 \mathrm{deg}$.

$-9<=\mathrm{h}<=9,-15<=\mathrm{k}<=15,-15<=\mathrm{k}<=15$

$5747 / 4387$ [R(int) $=0.0148]$

$100.0 \%$

Empirical

0.9545 and 0.9085

Full-matrix least-squares on $\mathrm{F}^{\wedge} 2$

4387 / 1 / 279

0.998

$\mathrm{R} 1=0.0359, \mathrm{wR} 2=0.0657$

$\mathrm{R} 1=0.0516, w \mathrm{R} 2=0.0689$

$-0.05(6)$

$0.0210(13)$

0.136 and -0.123 e. $\mathrm{A}^{\wedge}-3$ 
5. NMR, HRMS and HPLC spectra
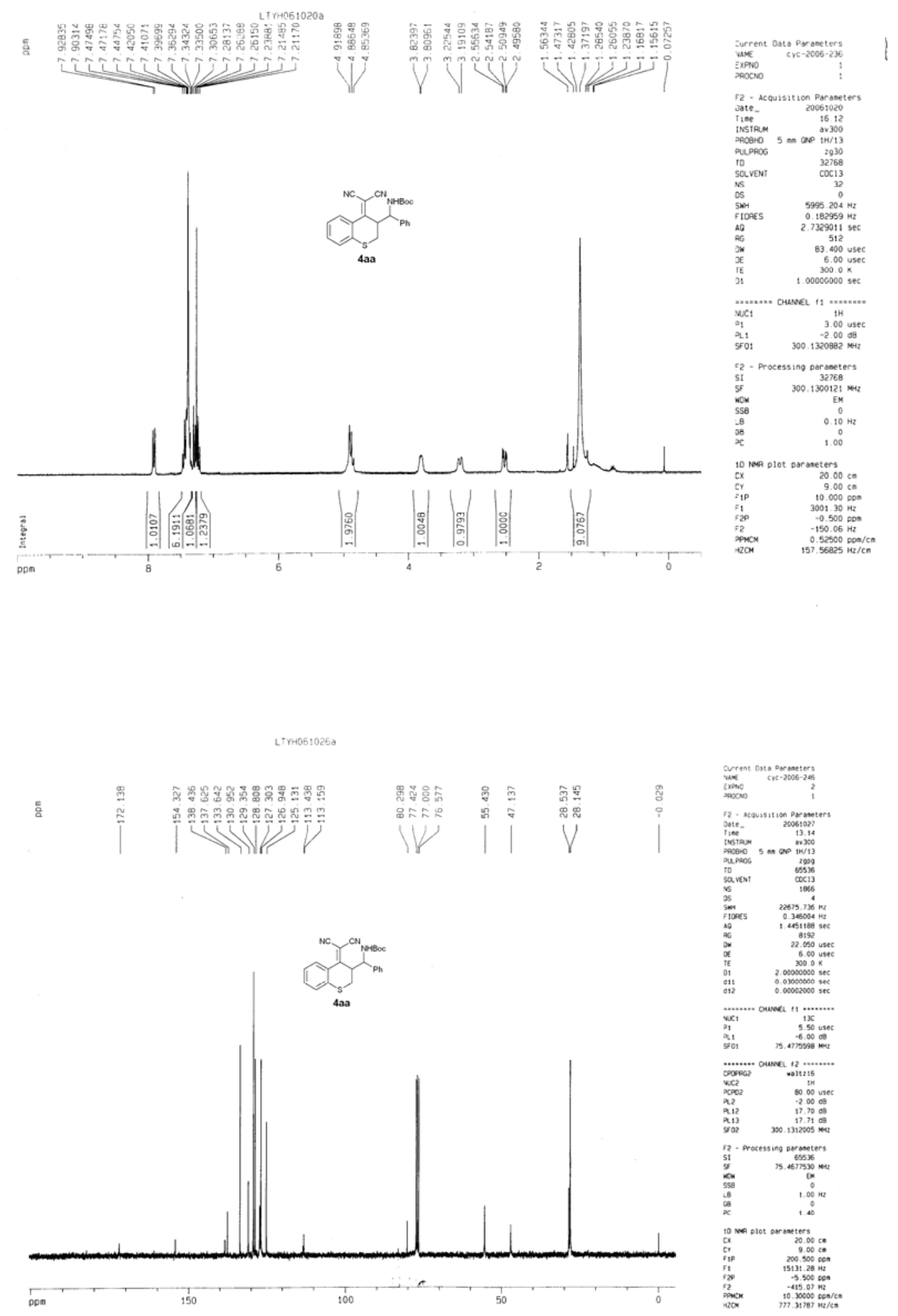


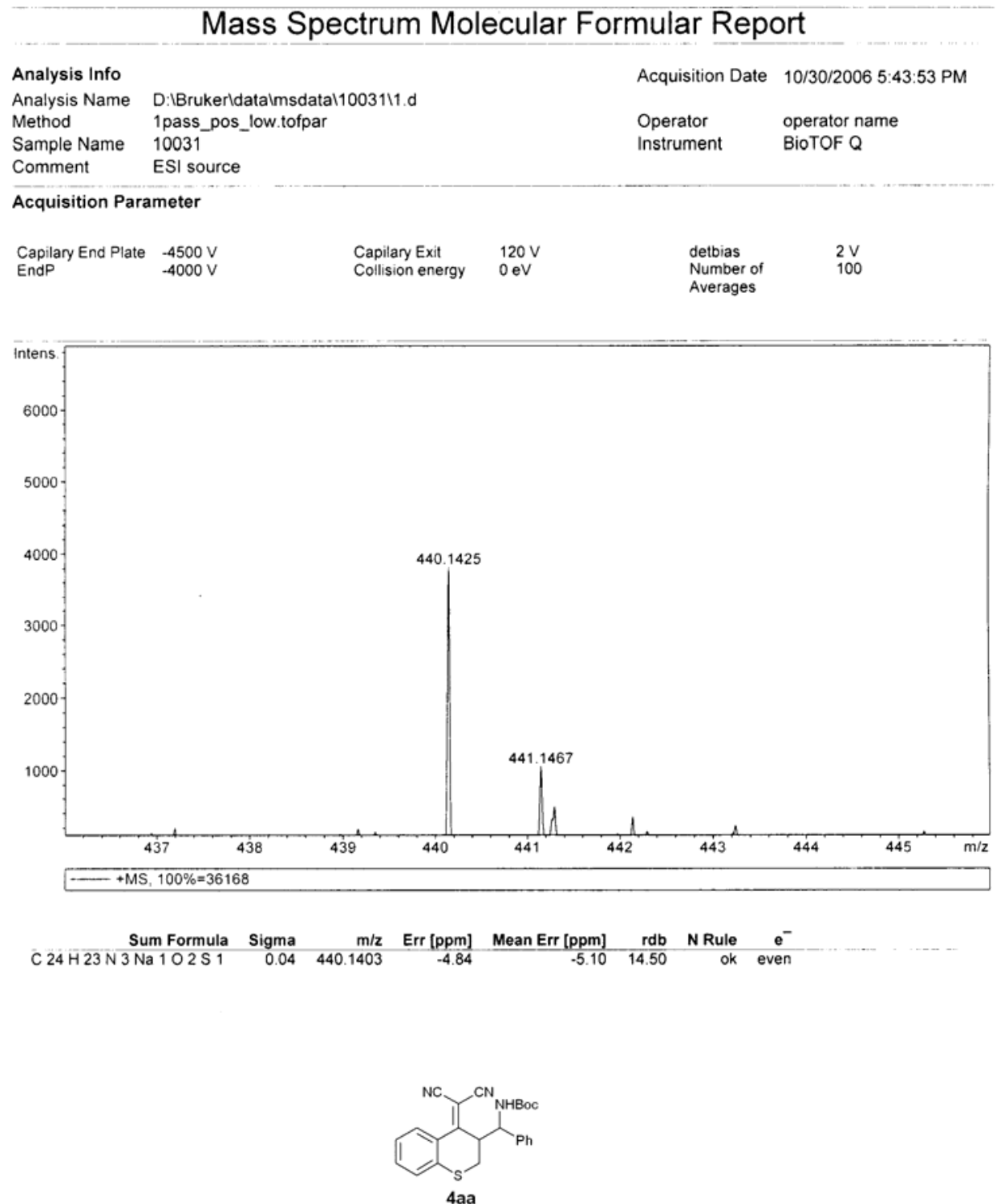




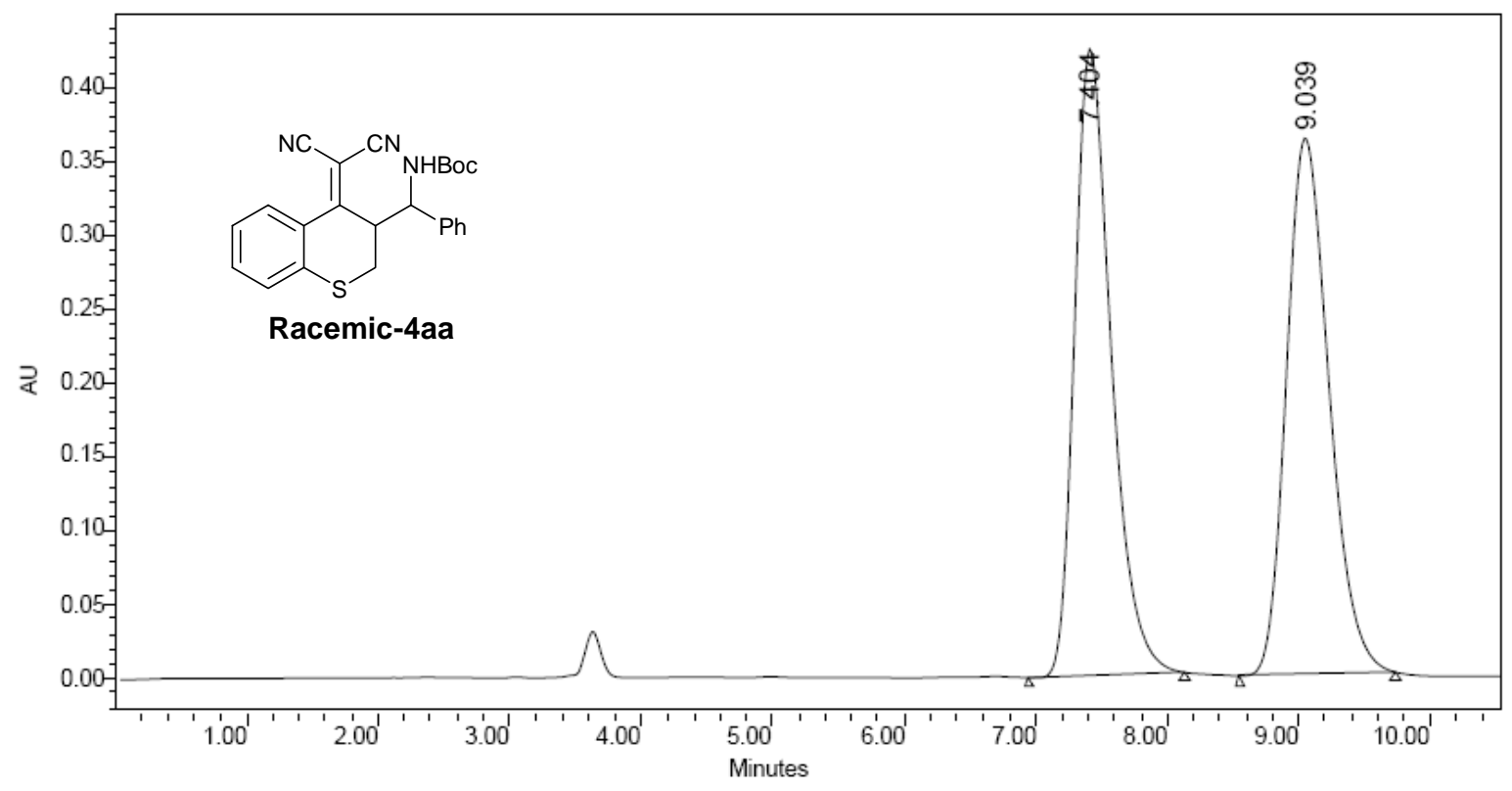

\begin{tabular}{|c|c|c|c|c|c|}
\hline & $\begin{array}{c}\text { RT } \\
(\mathrm{min})\end{array}$ & $\begin{array}{c}\text { Area } \\
\left(\mathrm{V}^{*} \mathrm{sec}\right)\end{array}$ & $\%$ Area & $\begin{array}{c}\text { Height } \\
(\mathrm{V})\end{array}$ & $\begin{array}{c}\% \\
\text { Height }\end{array}$ \\
\hline 1 & 7.404 & 8230126 & 50.08 & 424850 & 53.87 \\
\hline 2 & 9.039 & 8202207 & 49.92 & 363745 & 46.13 \\
\hline
\end{tabular}

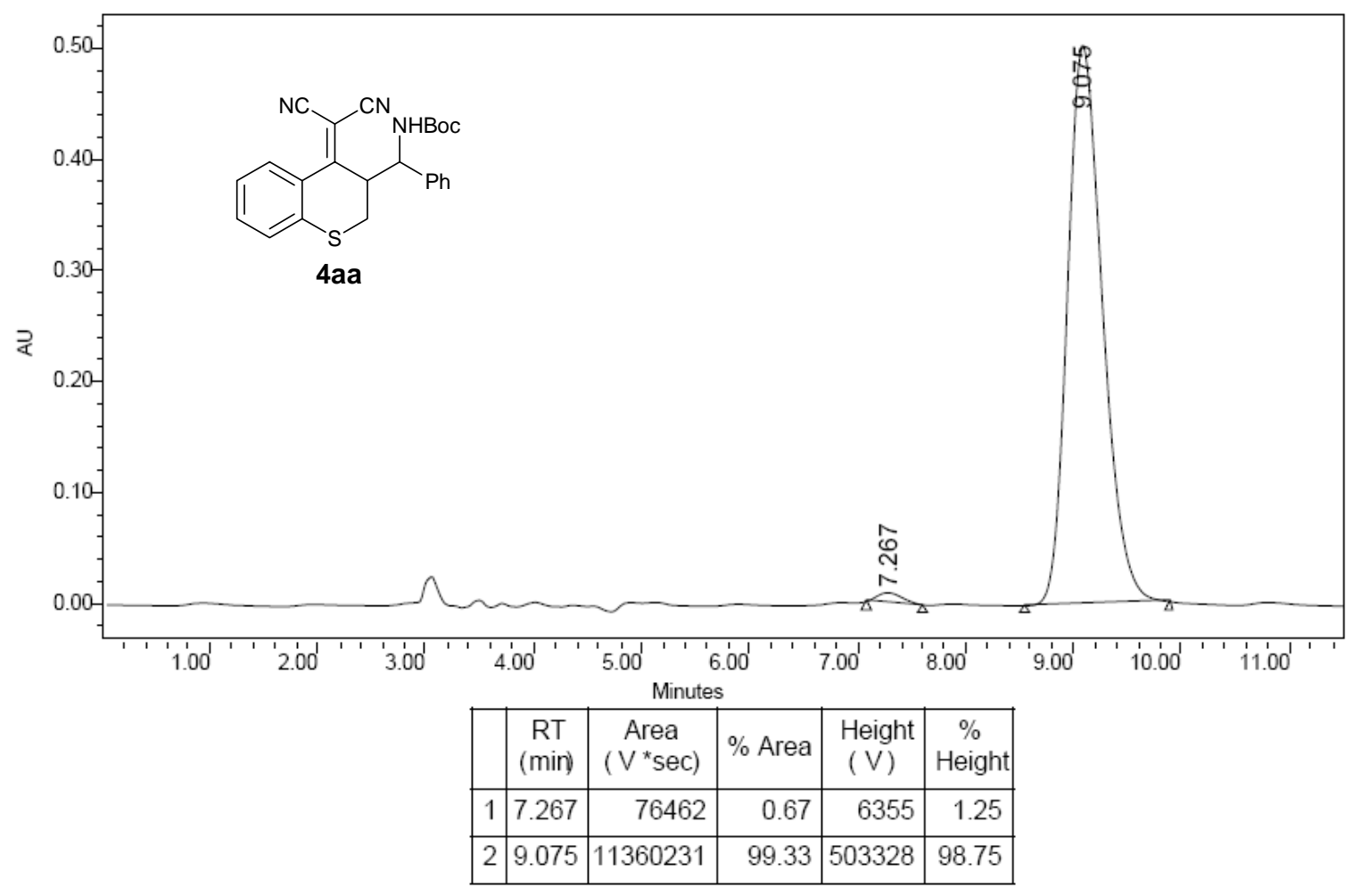




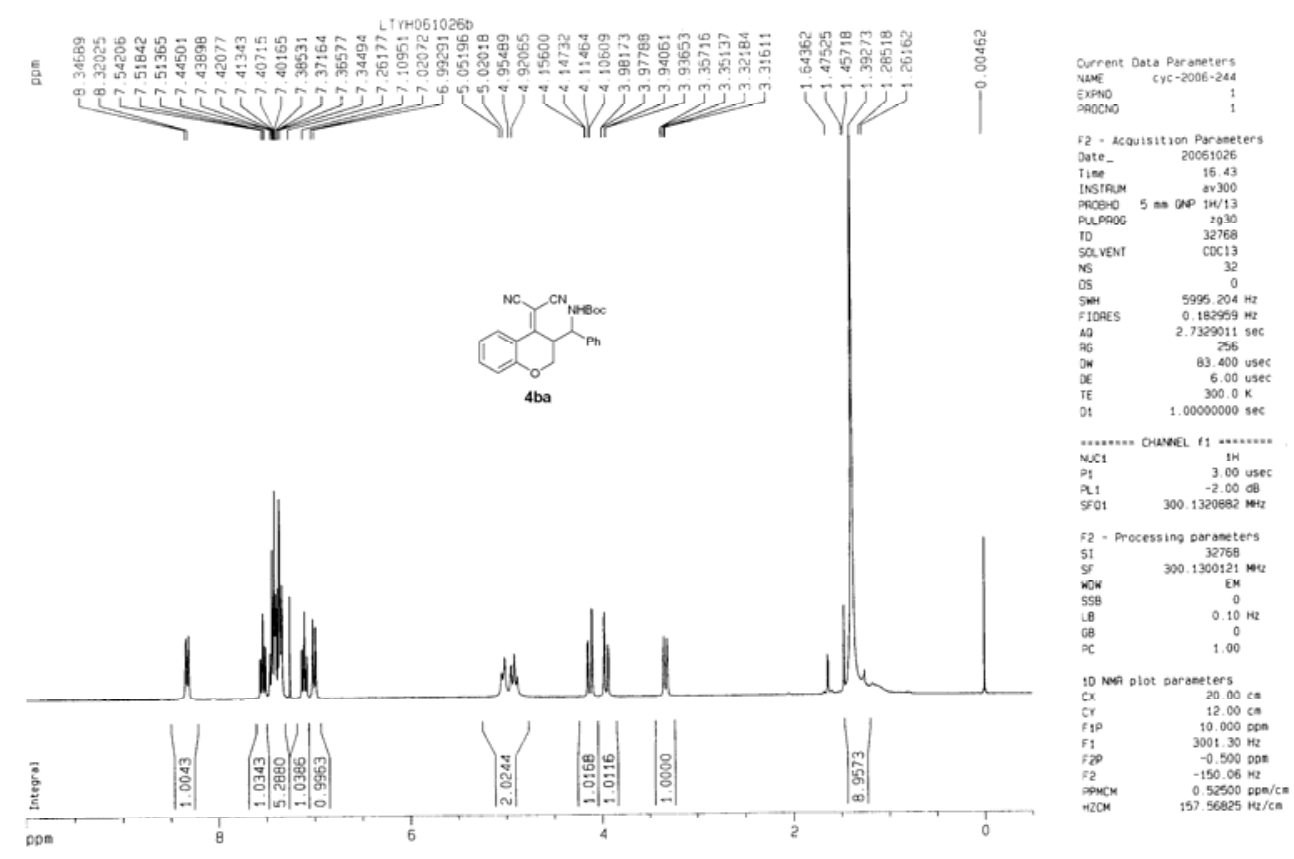

LTrM0610260

a

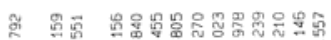

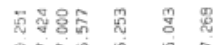

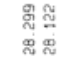

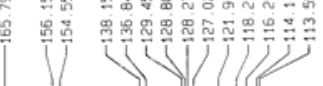

N| | |
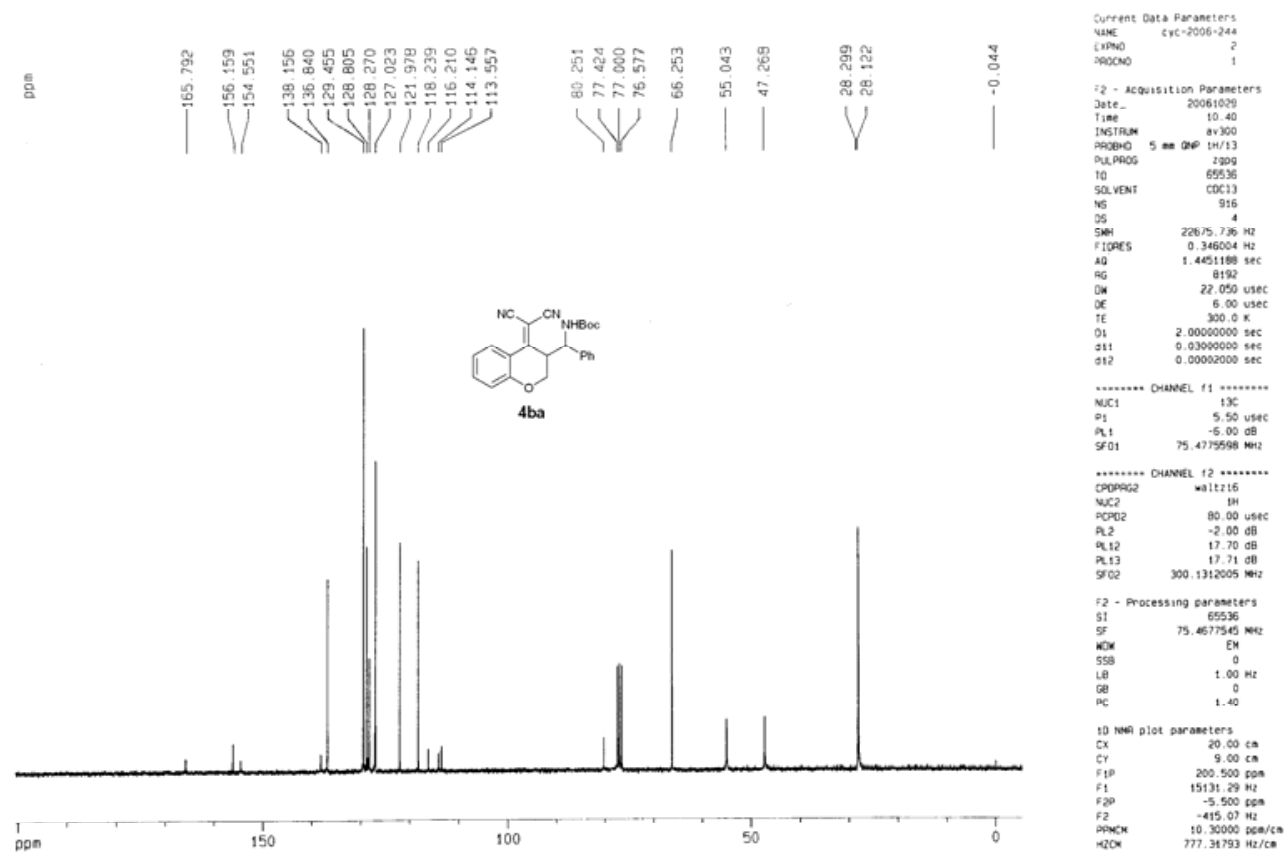


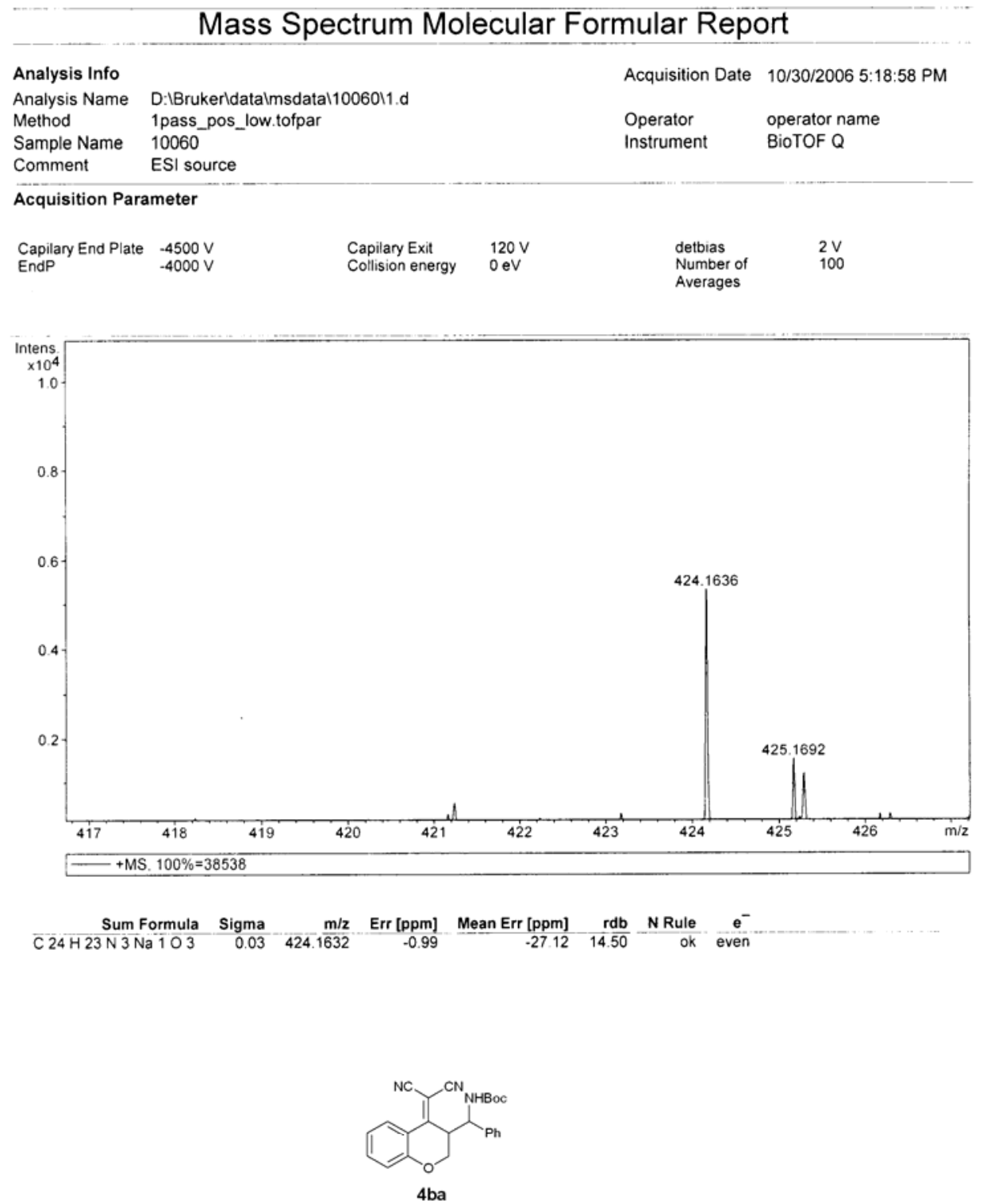

4ba 


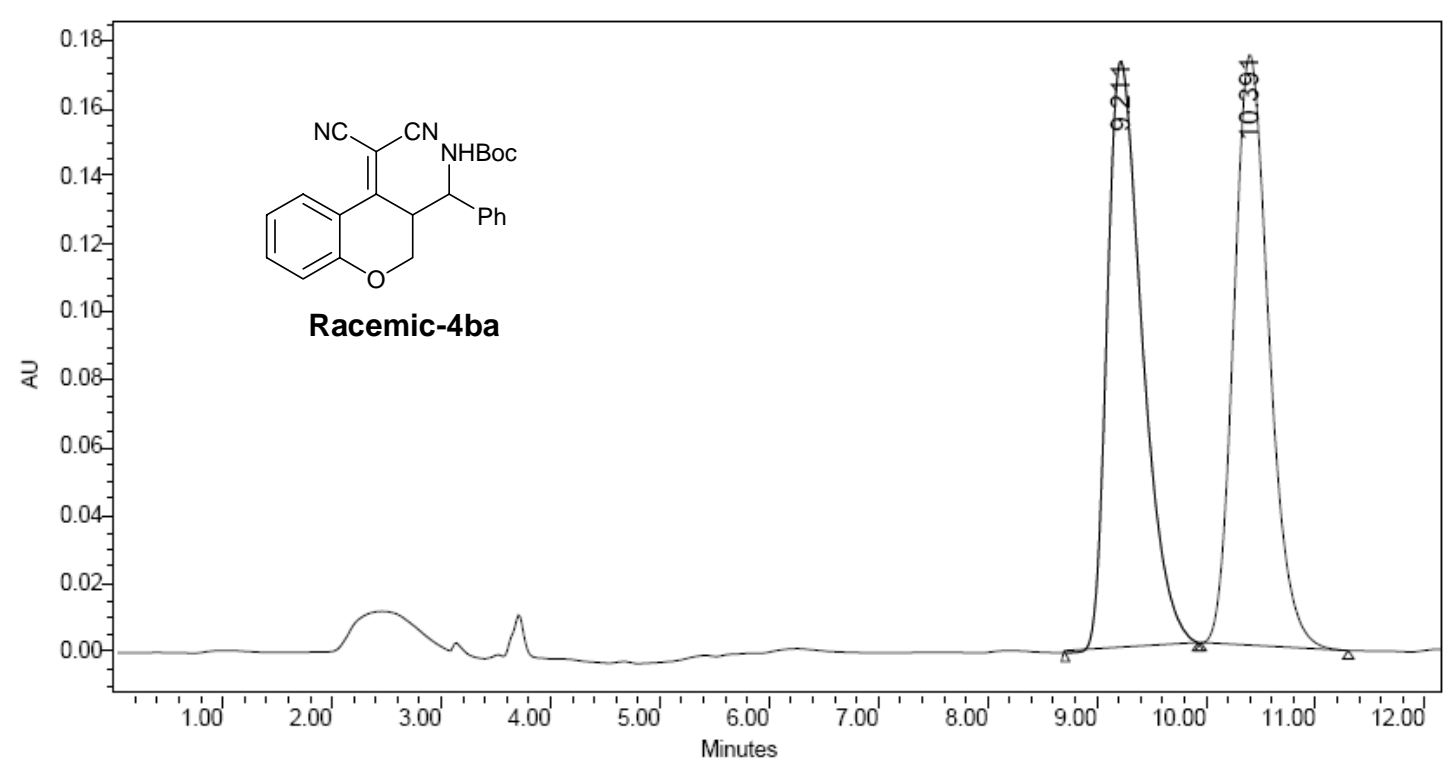

\begin{tabular}{|c|c|c|c|c|c|}
\hline & $\begin{array}{c}\text { RT } \\
(\mathrm{min})\end{array}$ & $\begin{array}{c}\text { Area } \\
\left(\mathrm{V}{ }^{*} \mathrm{sec}\right)\end{array}$ & $\%$ Area & $\begin{array}{c}\text { Height } \\
(\mathrm{V})\end{array}$ & $\begin{array}{c}\% \\
\text { Height }\end{array}$ \\
\hline 1 & 9.211 & 3865886 & 49.83 & 173133 & 49.85 \\
\hline 2 & 10.391 & 3892218 & 50.17 & 174165 & 50.15 \\
\hline
\end{tabular}

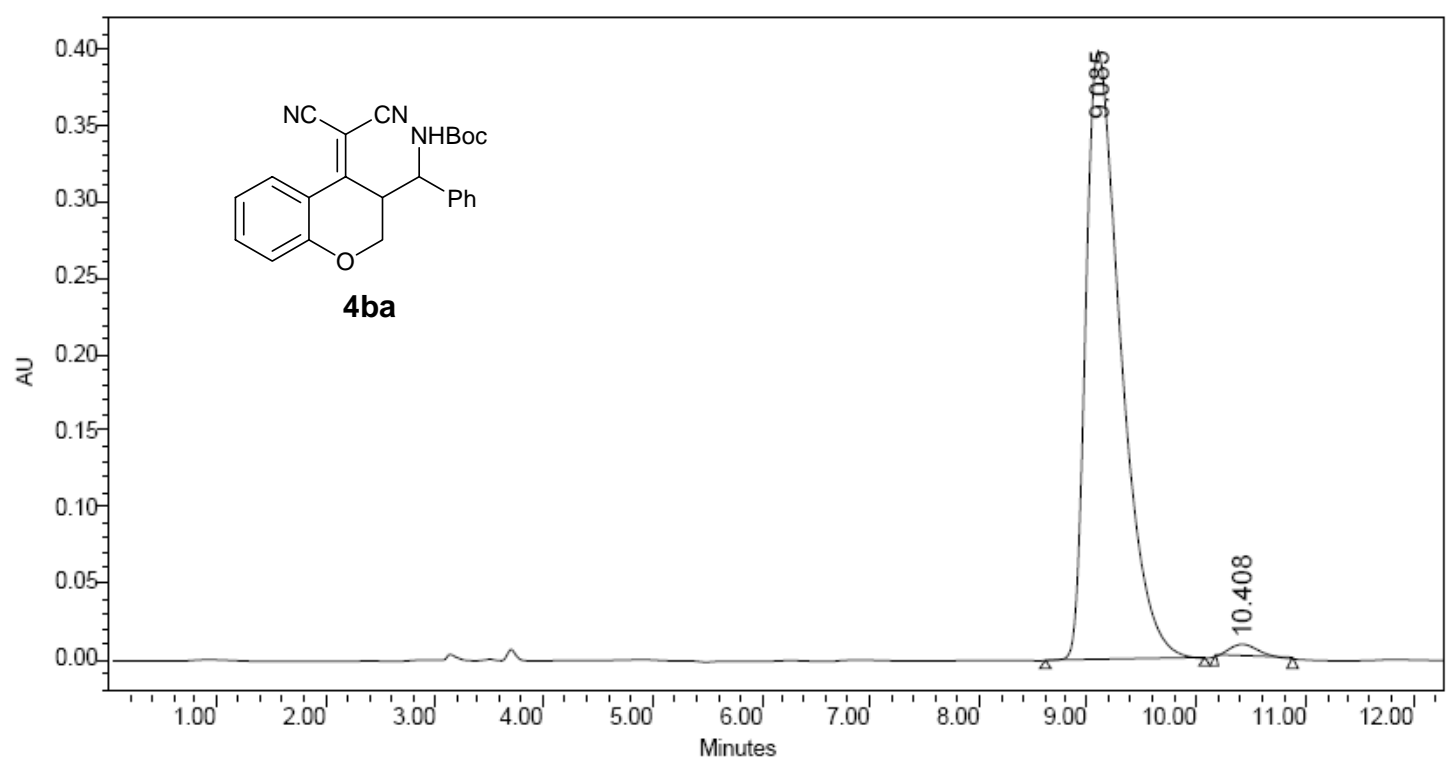

\begin{tabular}{|l|c|r|r|r|r|}
\hline & $\begin{array}{c}\text { RT } \\
(\text { min })\end{array}$ & $\begin{array}{c}\text { Area } \\
\left(\mathrm{V}^{*} \text { sec }\right)\end{array}$ & \% Area & $\begin{array}{c}\text { Height } \\
(\mathrm{V})\end{array}$ & $\begin{array}{c}\% \\
\text { Height }\end{array}$ \\
\hline 1 & 9.085 & 8908217 & 99.10 & 399103 & 98.55 \\
\hline 2 & 10.408 & 80656 & 0.90 & 5890 & 1.45 \\
\hline
\end{tabular}



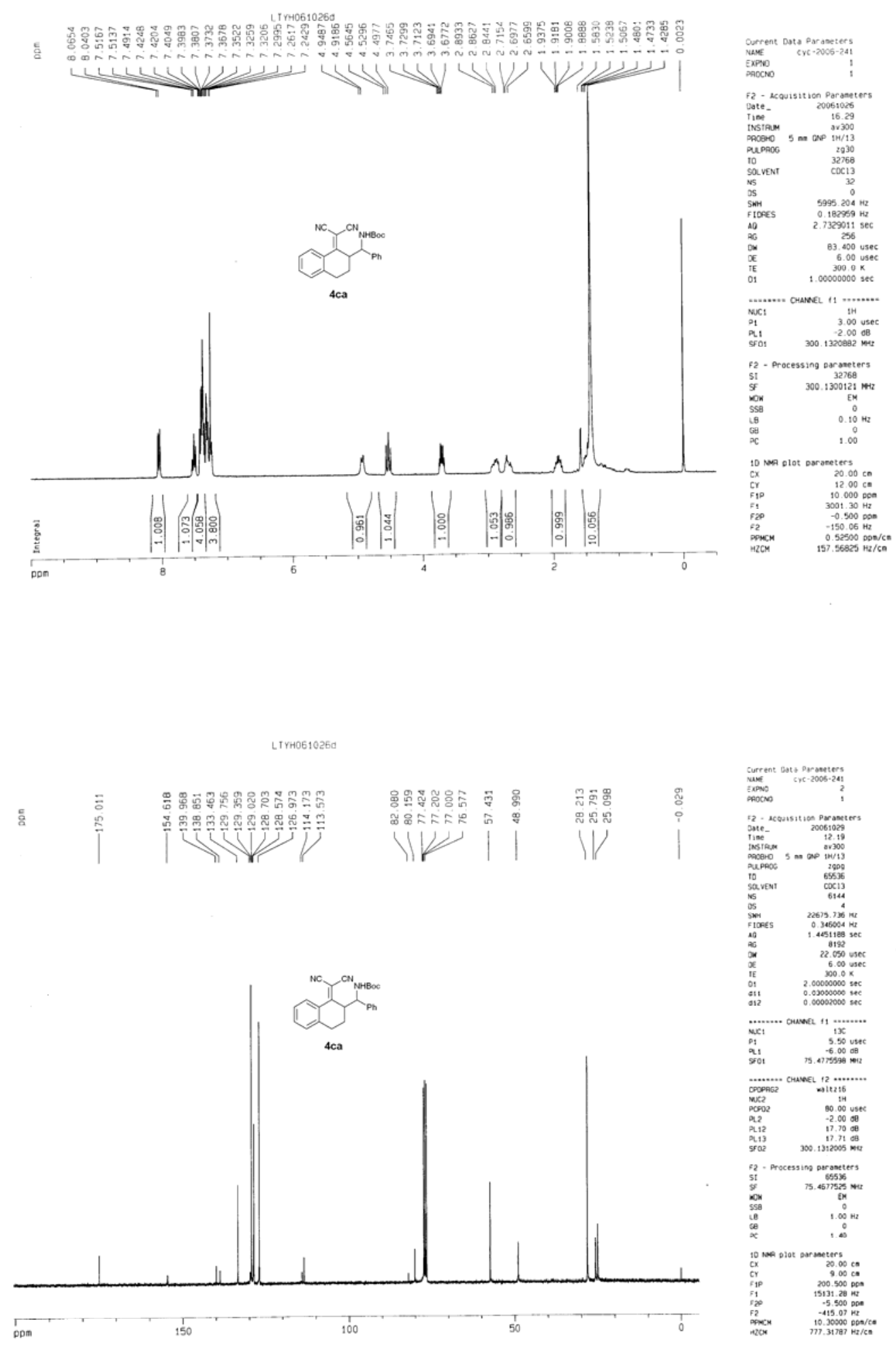
Mass Spectrum Molecular Formular Report

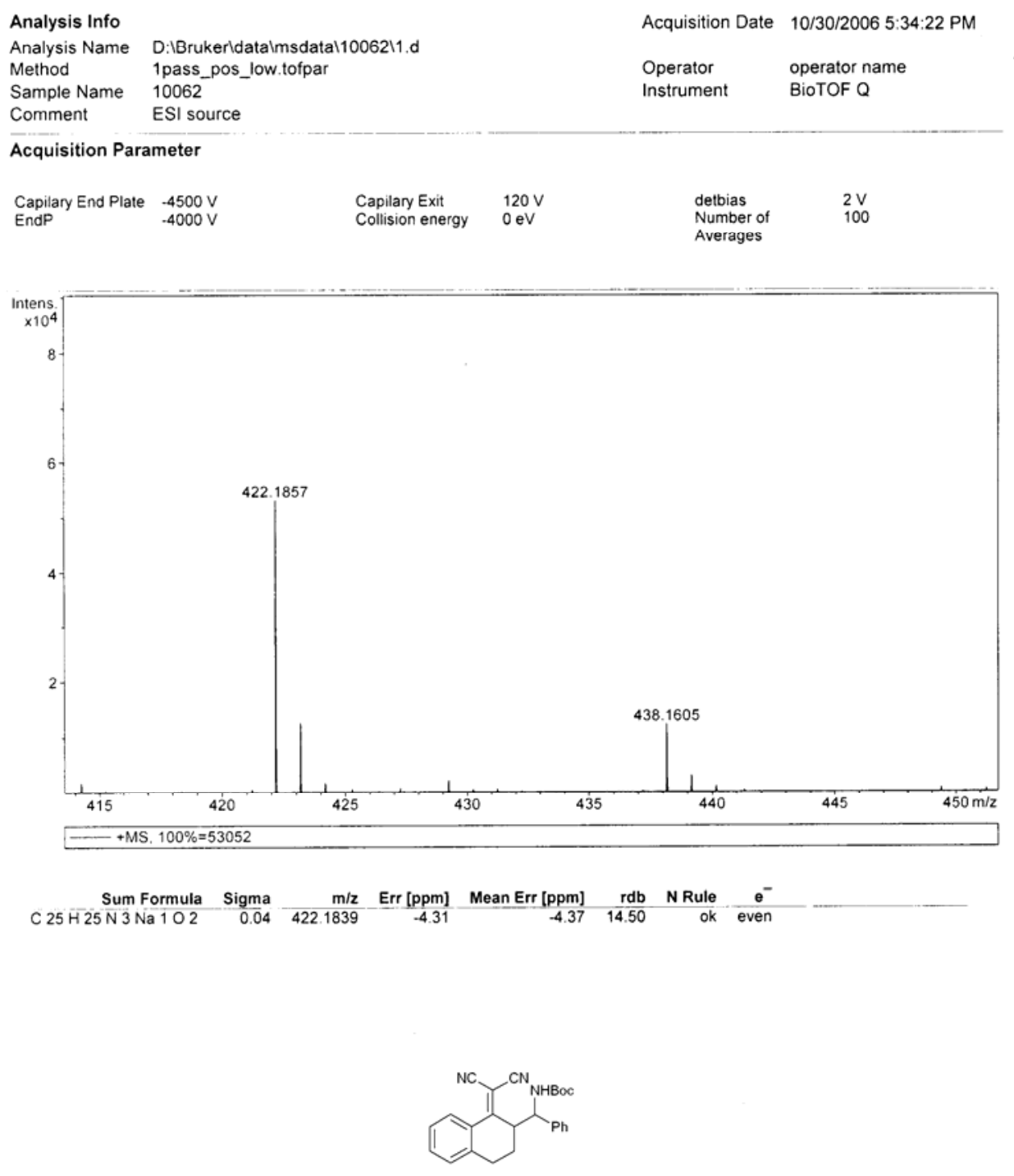

$4 \mathrm{ca}$ 


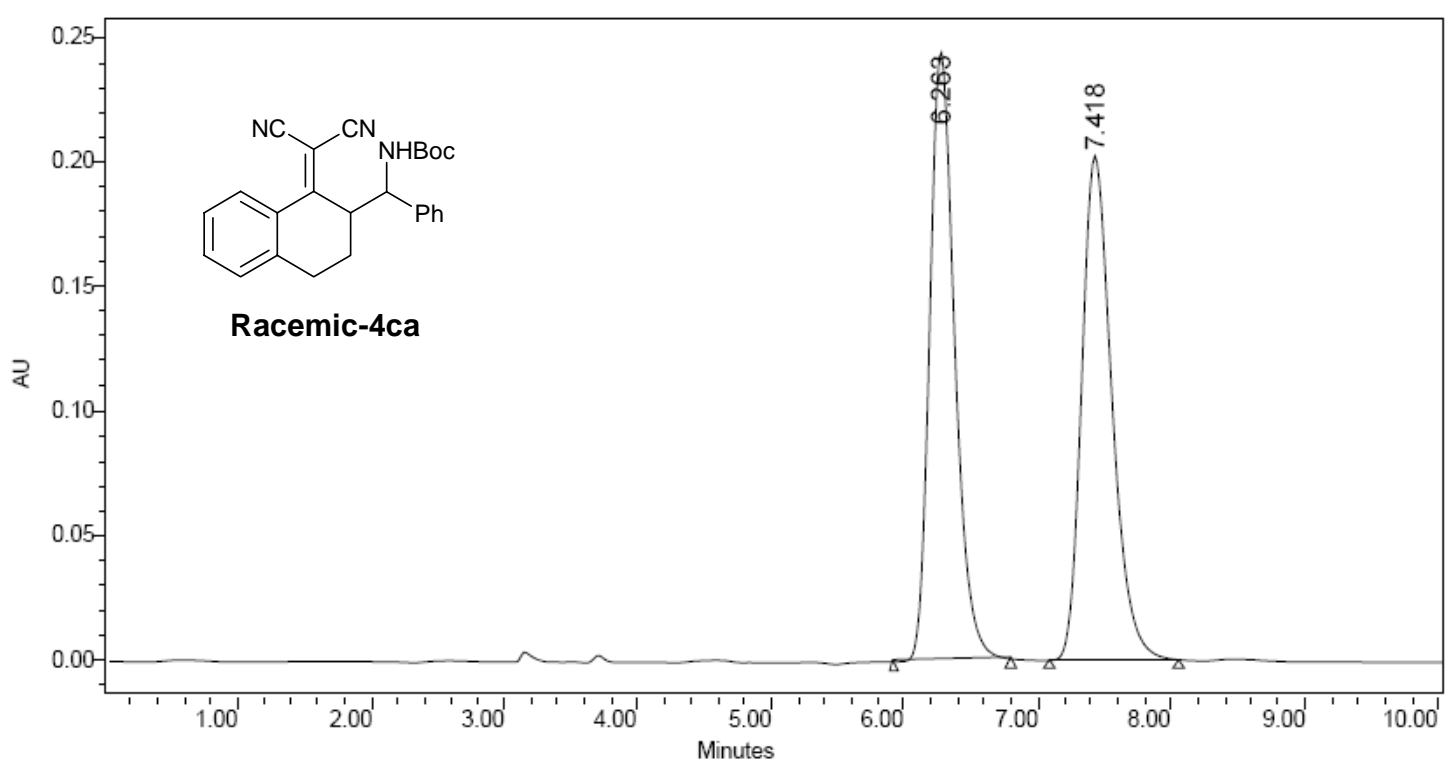

\begin{tabular}{|c|c|c|c|c|c|}
\hline & $\begin{array}{c}\text { RT } \\
(\mathrm{min})\end{array}$ & $\begin{array}{c}\text { Area } \\
\left(\mathrm{V}^{*} \mathrm{sec}\right)\end{array}$ & $\%$ Area & $\begin{array}{c}\text { Height } \\
(\mathrm{V})\end{array}$ & $\begin{array}{c}\% \\
\text { Height }\end{array}$ \\
\hline 1 & 6.263 & 3190351 & 50.09 & 244306 & 54.69 \\
\hline 2 & 7.418 & 3178665 & 49.91 & 202390 & 45.31 \\
\hline
\end{tabular}

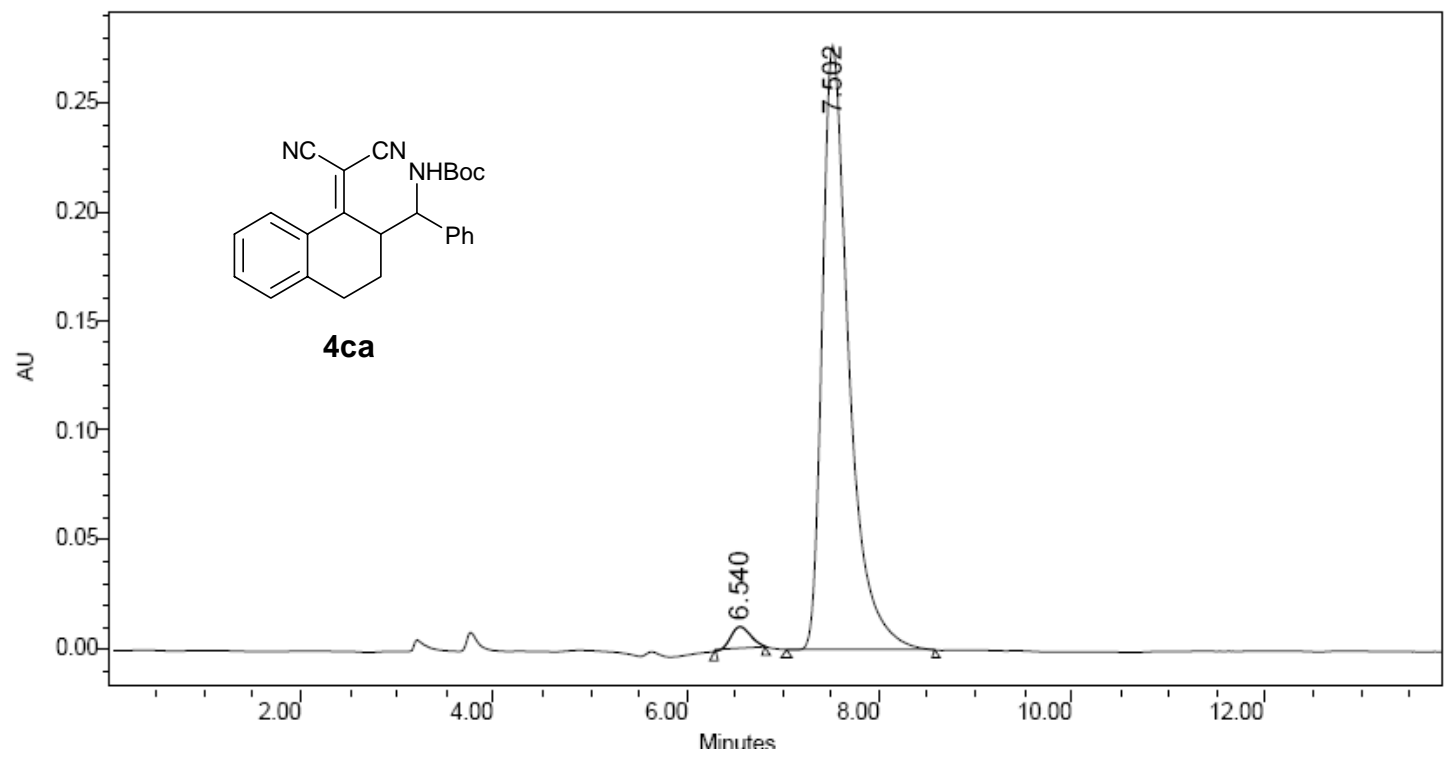

\begin{tabular}{|l|c|r|r|r|r|}
\hline & $\begin{array}{c}\mathrm{RT} \\
(\mathrm{min})\end{array}$ & $\begin{array}{c}\text { Area } \\
\left(\mathrm{V}{ }^{*} \mathrm{sec}\right)\end{array}$ & $\%$ Area & $\begin{array}{c}\text { Height } \\
(\mathrm{V})\end{array}$ & $\begin{array}{c}\% \\
\text { Height }\end{array}$ \\
\hline 1 & 6.540 & 82843 & 1.53 & 7556 & 2.66 \\
\hline 2 & 7.502 & 5332757 & 98.47 & 276012 & 97.34 \\
\hline
\end{tabular}




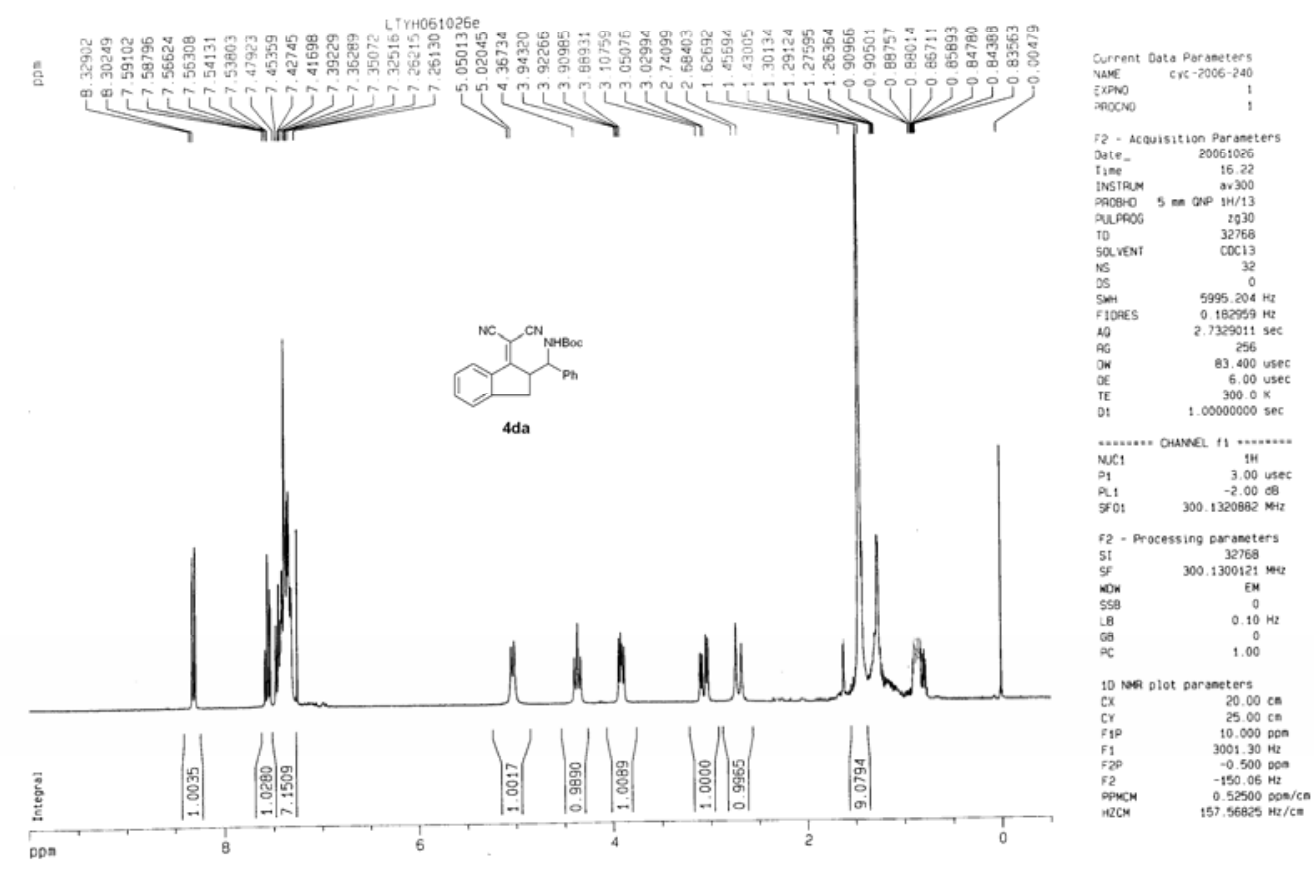

LTYH061026e

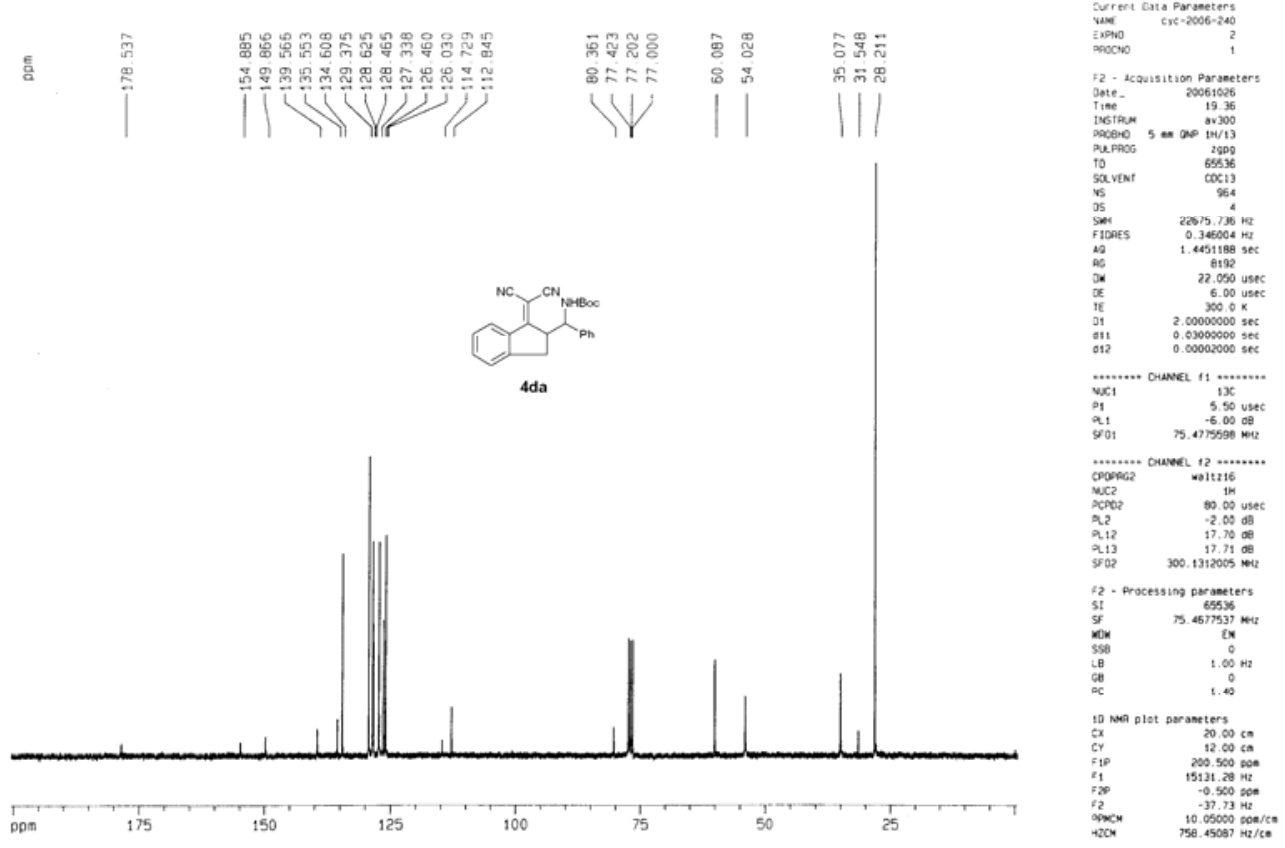




\section{Mass Spectrum Molecular Formular Report}

\section{Analysis Info}

Analysis Name D:IBrukerldatalmsdatal1006311.d

Method

pass_pos_low.tofpar

Acquisition Date 10/30/2006 5:09:47 PM

Sample Name

10063

Operator operator name

Comment

ESI source

Instrument

BioTOF Q

Acquisition Parameter

$\begin{array}{ll}\text { Capilary End Plate } & -4500 \mathrm{~V} \\ \text { EndP } & -4000 \mathrm{~V}\end{array}$

Capilary Exit $120 \mathrm{~V}$

$\begin{array}{ll}\text { detbias } & 2 \mathrm{~V} \\ \text { Number of } & 100\end{array}$

Collision energy $0 \mathrm{eV}$

Averages

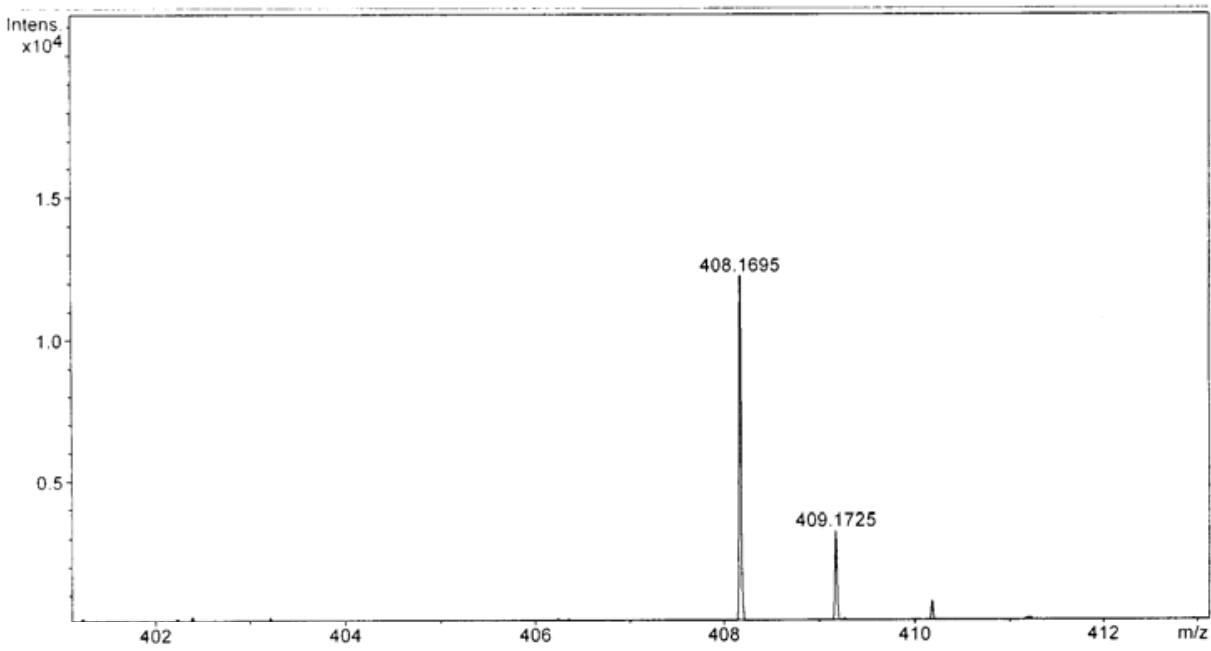

+ MS $100 \%=2675$

Sum Formula Sigma $\mathrm{m} / \mathrm{z} \quad \mathrm{Err}[\mathrm{ppm}] \quad$ Mean Err [ppm] $\mathrm{rdb} \quad \mathrm{N}$ Rule

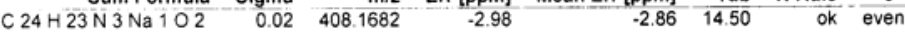

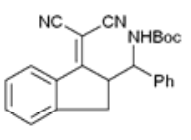

4da 


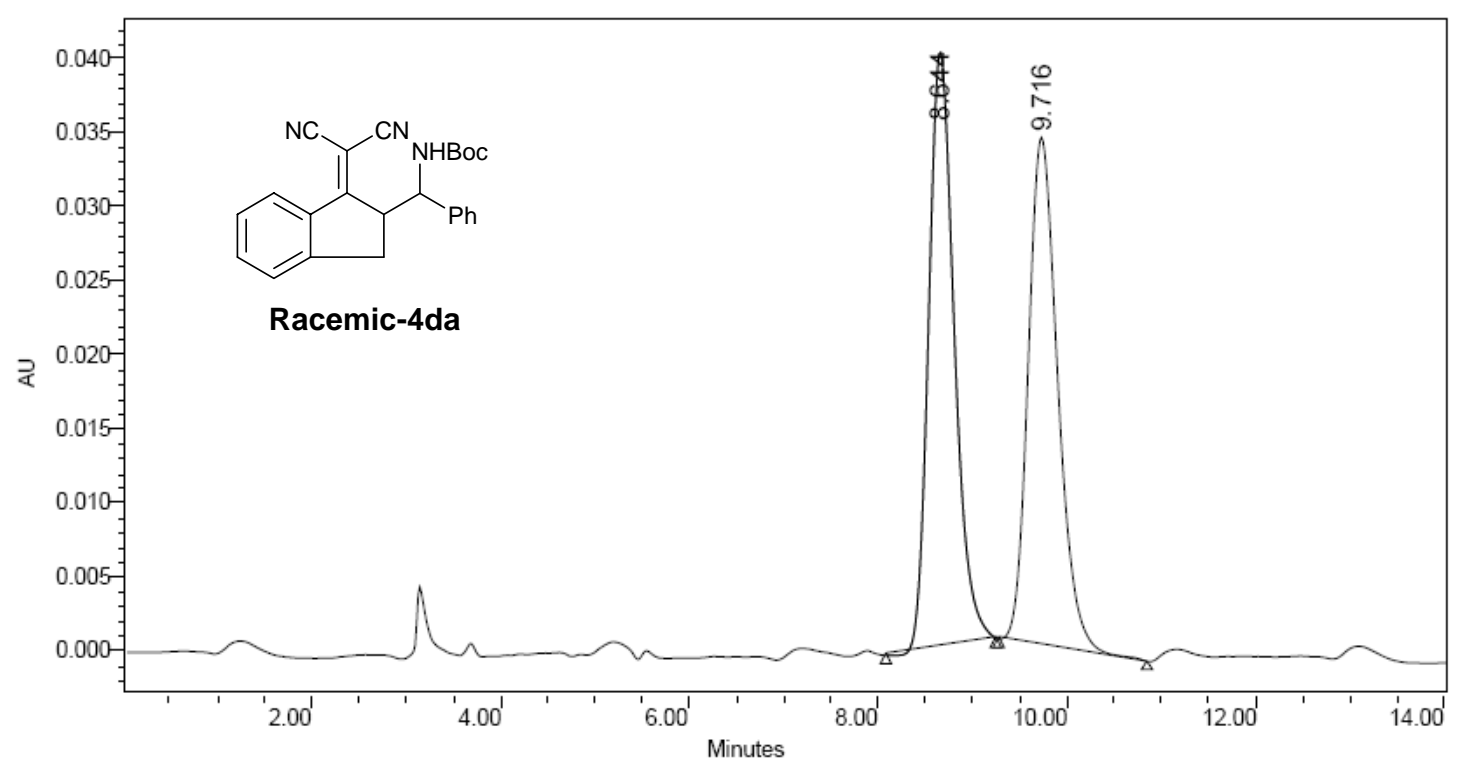

\begin{tabular}{|c|c|c|c|c|c|}
\hline & $\begin{array}{c}\text { RT } \\
(\text { min })\end{array}$ & $\begin{array}{c}\text { Area } \\
\left(V^{*} \text { sec }\right)\end{array}$ & $\%$ Area & $\begin{array}{c}\text { Height } \\
(\mathrm{V})\end{array}$ & $\begin{array}{c}\% \\
\text { Height }\end{array}$ \\
\hline 1 & 8.644 & 760767 & 50.45 & 40222 & 53.96 \\
\hline 2 & 9.716 & 747180 & 49.55 & 34313 & 46.04 \\
\hline
\end{tabular}

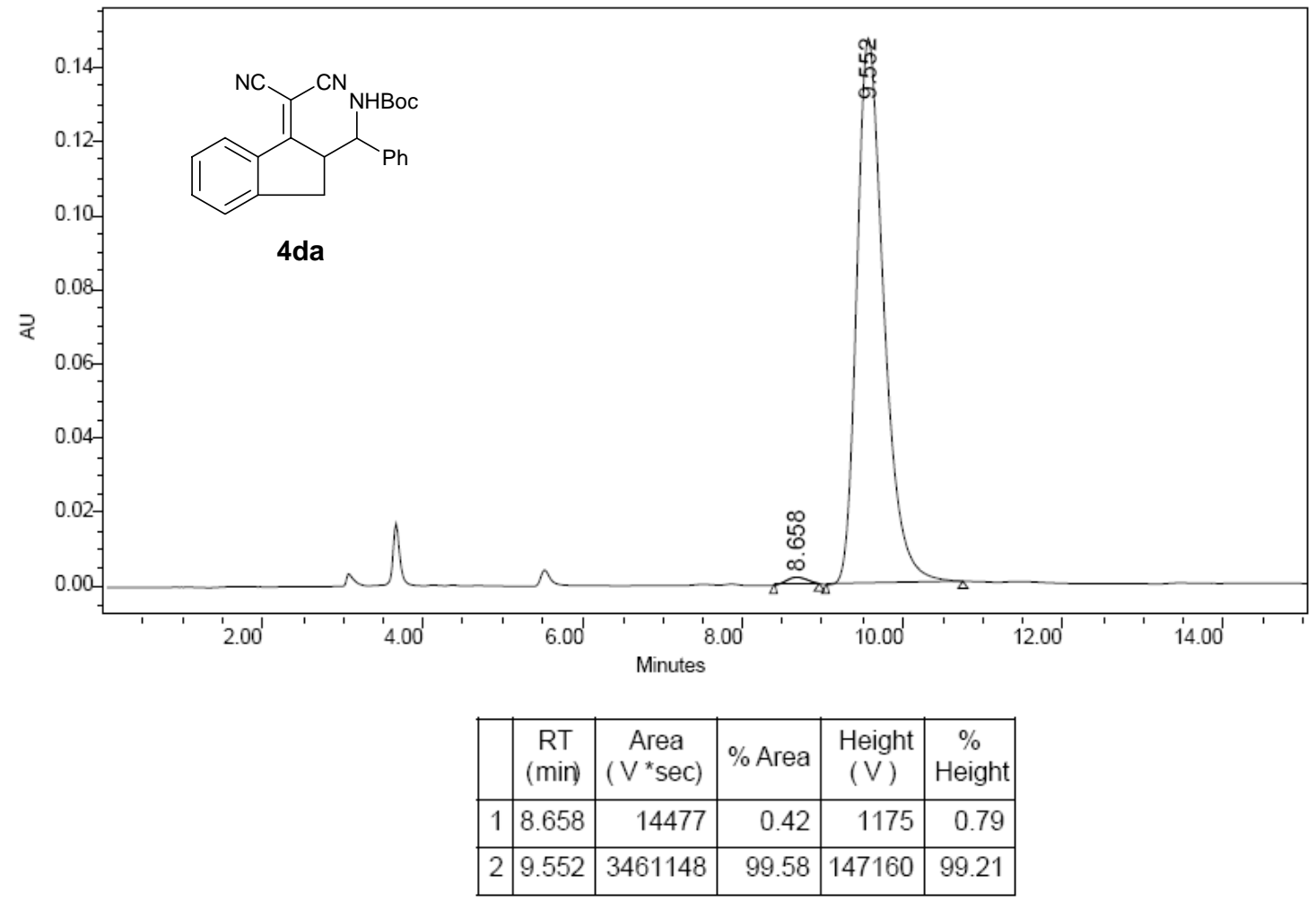




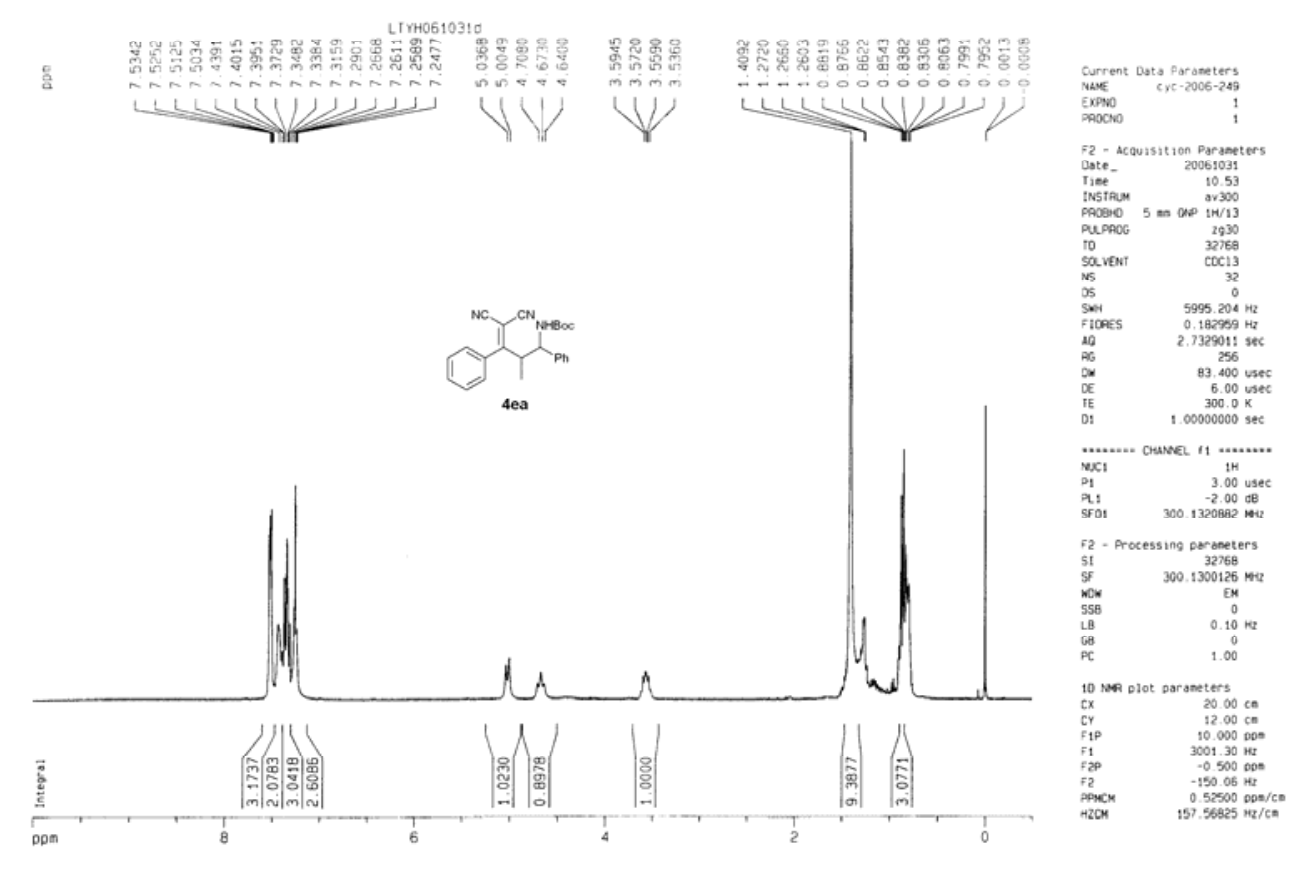

LTYHO6:03!

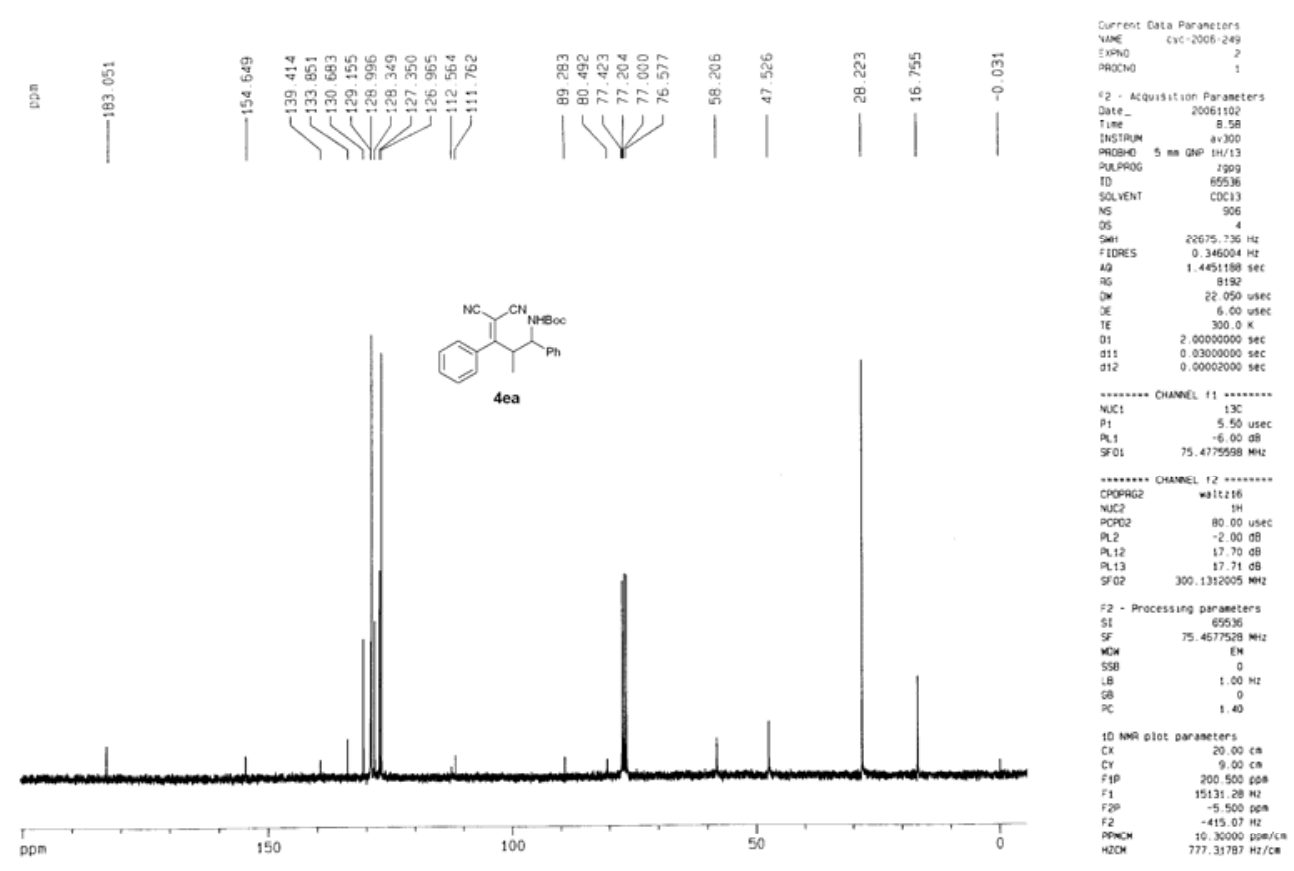




\section{Mass Spectrum Molecular Formular Report}

Analysis Info

Analysis Name D:IBrukerldatalmsdatal1007111.d

Method

Sample Name

Comment

1pass_pos_low.tofpar

10071

ESI source

\section{Acquisition Parameter}

Capilary End Plate $-4500 \mathrm{~V}$ $-4000 \mathrm{~V}$

Capilary Exit $\quad 120 \mathrm{~V}$

$120 \mathrm{~V}$
$0 \mathrm{eV}$

detbias

Number of

Averages

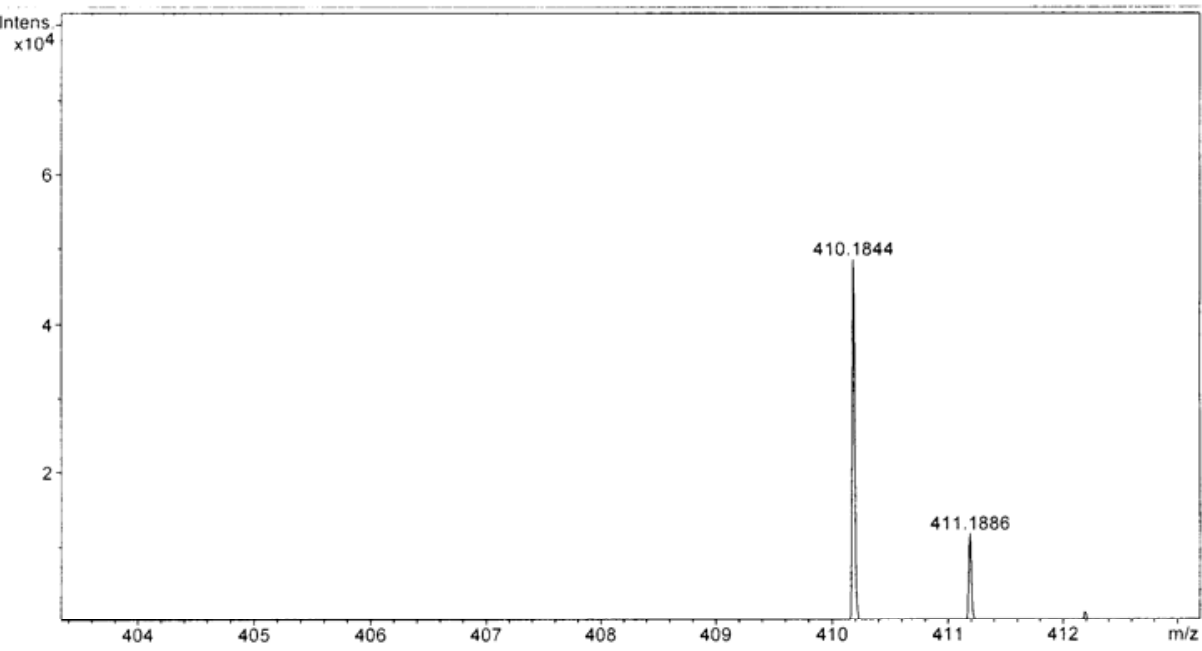

$-+M S, 100 \%=48540$

Sum Formula Sigma $\mathrm{m} / \mathbf{z}$ Err [ppm] Mean Err [ppm] rdb NRule $e^{-}$
Acquisition Date 10/30/2006 5:05:53 PM

Operator name

Instrument BioTOF Q

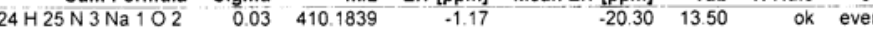

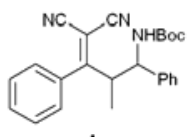

4ea 


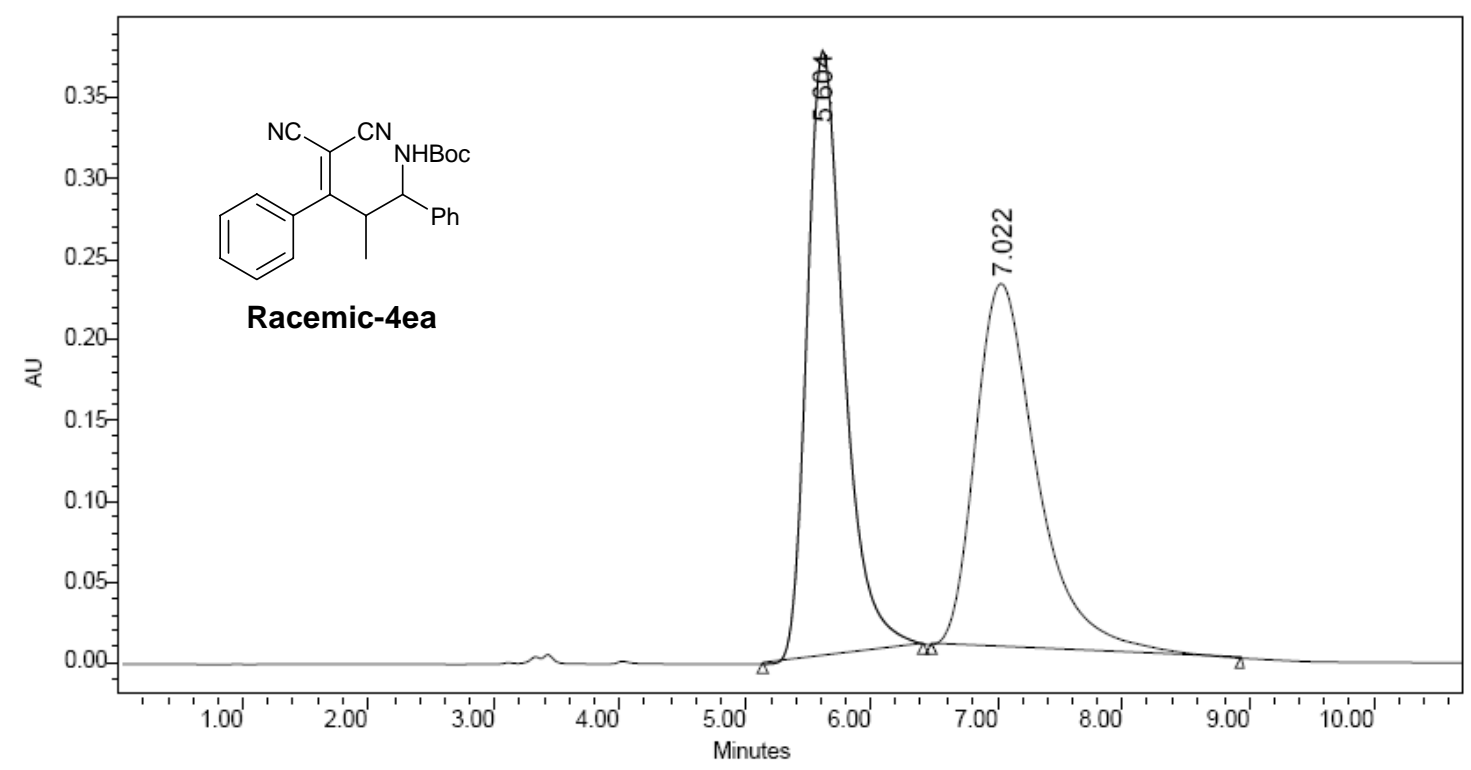

\begin{tabular}{|c|c|c|c|c|c|}
\hline & $\begin{array}{c}\text { RT } \\
(\mathrm{min})\end{array}$ & $\begin{array}{c}\text { Area } \\
\left(\mathrm{V}^{*} \mathrm{sec}\right)\end{array}$ & $\%$ Area & $\begin{array}{c}\text { Height } \\
(\mathrm{V})\end{array}$ & $\begin{array}{c}\% \\
\text { Height }\end{array}$ \\
\hline 1 & 5.604 & 7678805 & 49.77 & 375512 & 62.49 \\
\hline 2 & 7.022 & 7748989 & 50.23 & 225450 & 37.51 \\
\hline
\end{tabular}

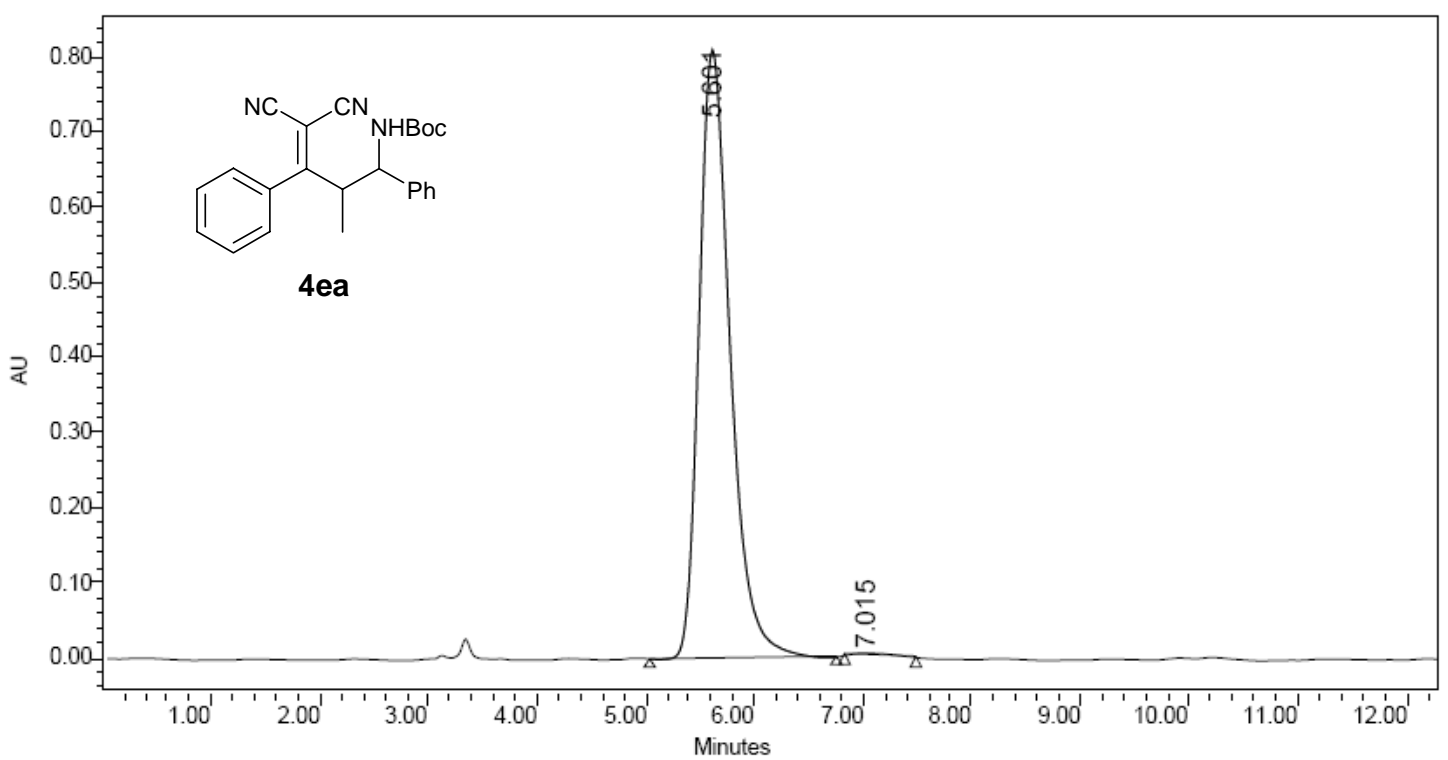

\begin{tabular}{|l|c|r|r|r|r|}
\hline & $\begin{array}{c}\text { RT } \\
(\mathrm{min})\end{array}$ & $\begin{array}{c}\text { Area } \\
(\mathrm{V} \text { *sec })\end{array}$ & $\%$ Area & $\begin{array}{c}\text { Height } \\
(\mathrm{V})\end{array}$ & $\begin{array}{c}\% \\
\text { Height }\end{array}$ \\
\hline 1 & 5.601 & 16139016 & 99.36 & 810471 & 99.49 \\
\hline 2 & 7.015 & 103851 & 0.64 & 4127 & 0.51 \\
\hline
\end{tabular}



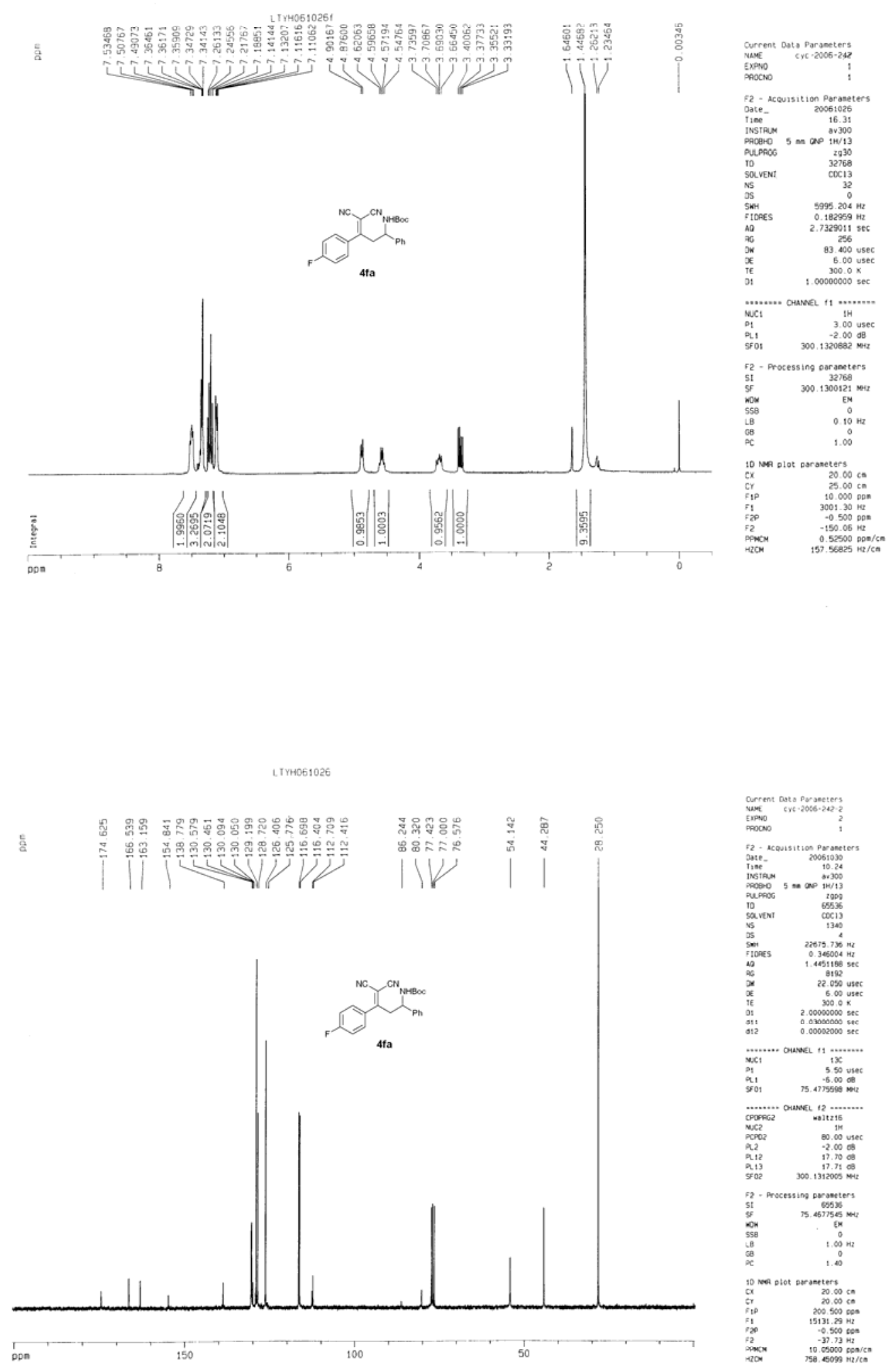


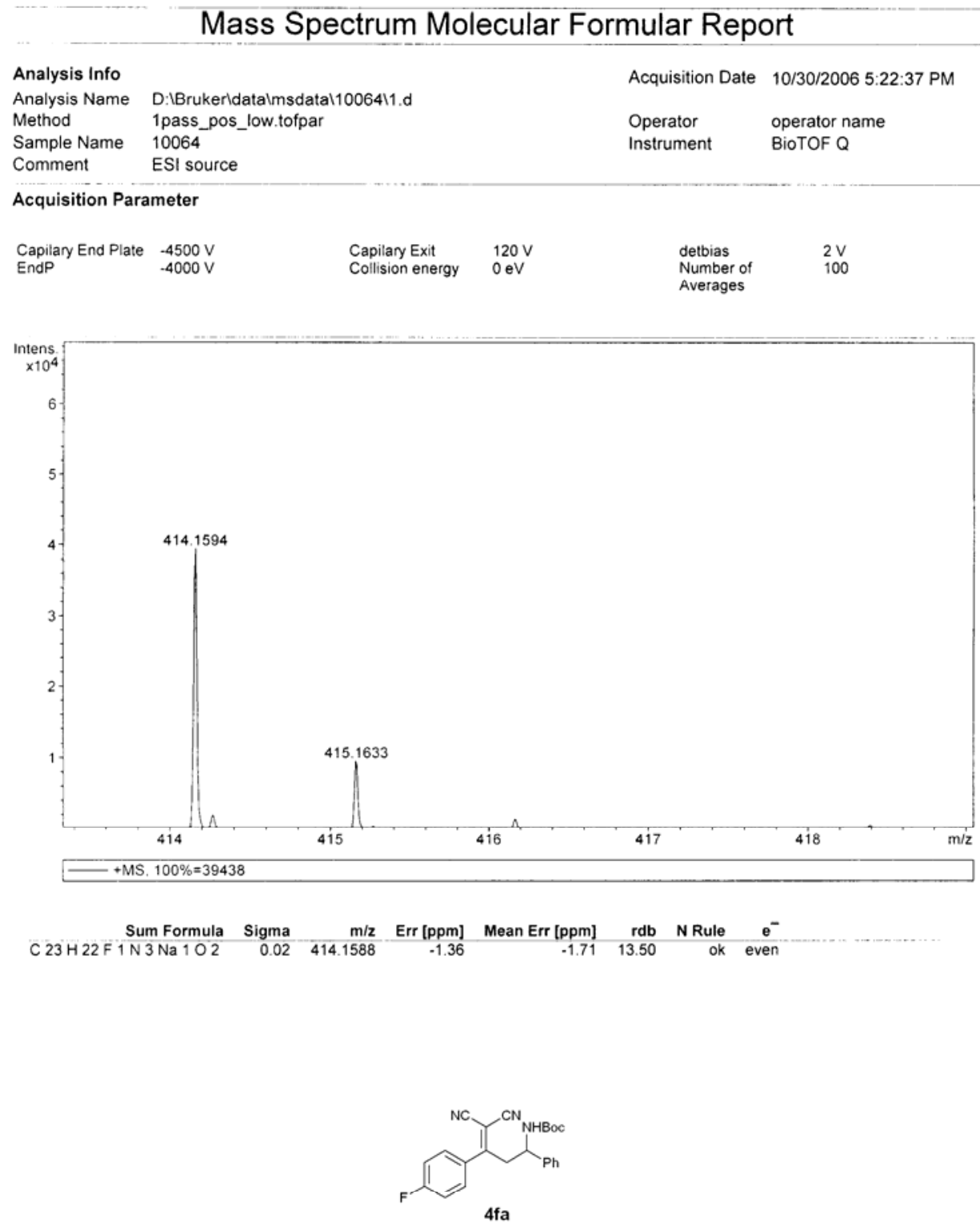

$4 f a$ 


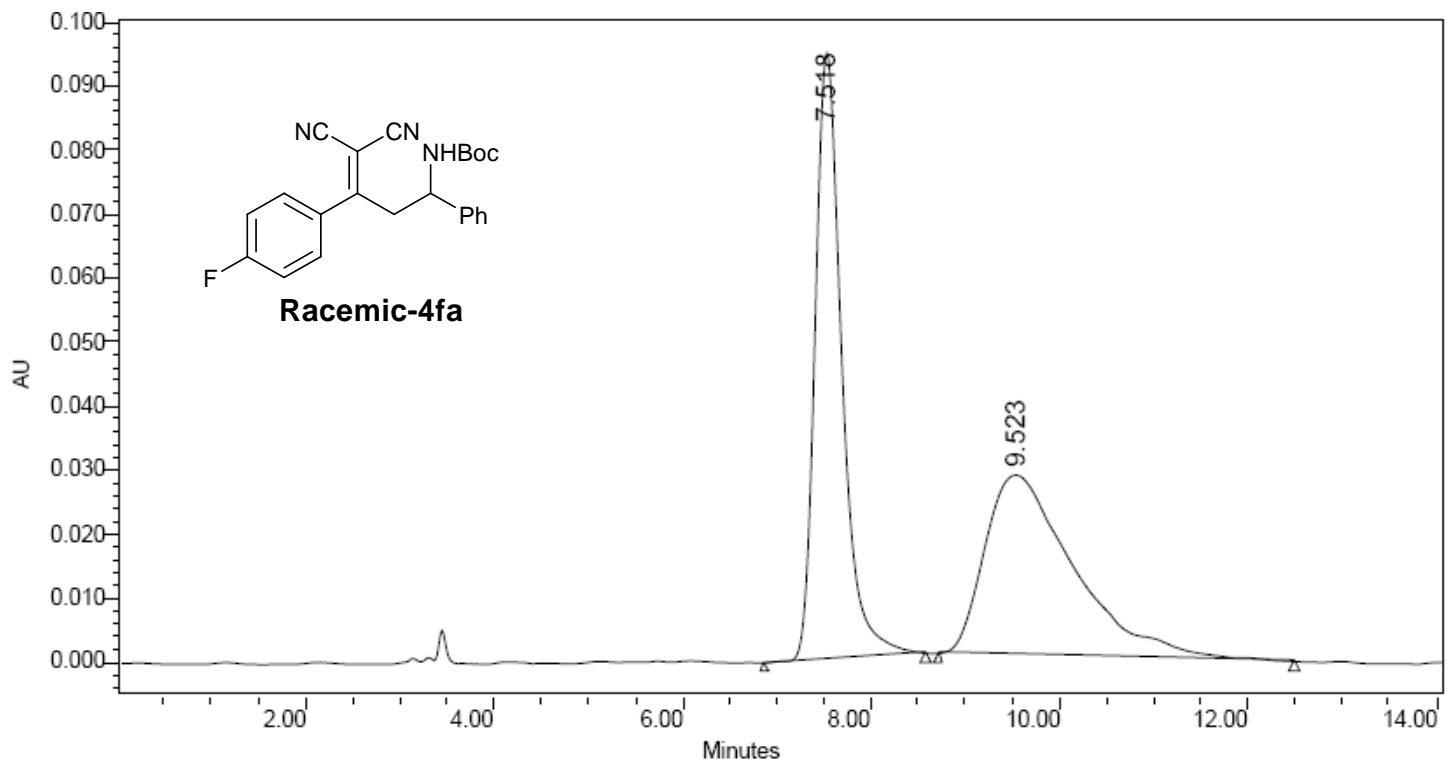

\begin{tabular}{|c|c|c|c|c|c|}
\hline & $\begin{array}{c}\mathrm{RT} \\
(\mathrm{min})\end{array}$ & $\begin{array}{c}\text { Area } \\
\left(\mathrm{V}{ }^{*} \mathrm{sec}\right)\end{array}$ & $\%$ Area & $\begin{array}{c}\text { Height } \\
(\mathrm{V})\end{array}$ & $\begin{array}{c}\% \\
\text { Height }\end{array}$ \\
\hline 1 & 7.518 & 1871655 & 50.15 & 94669 & 77.10 \\
\hline 2 & 9.523 & 1860403 & 49.85 & 28112 & 22.90 \\
\hline
\end{tabular}

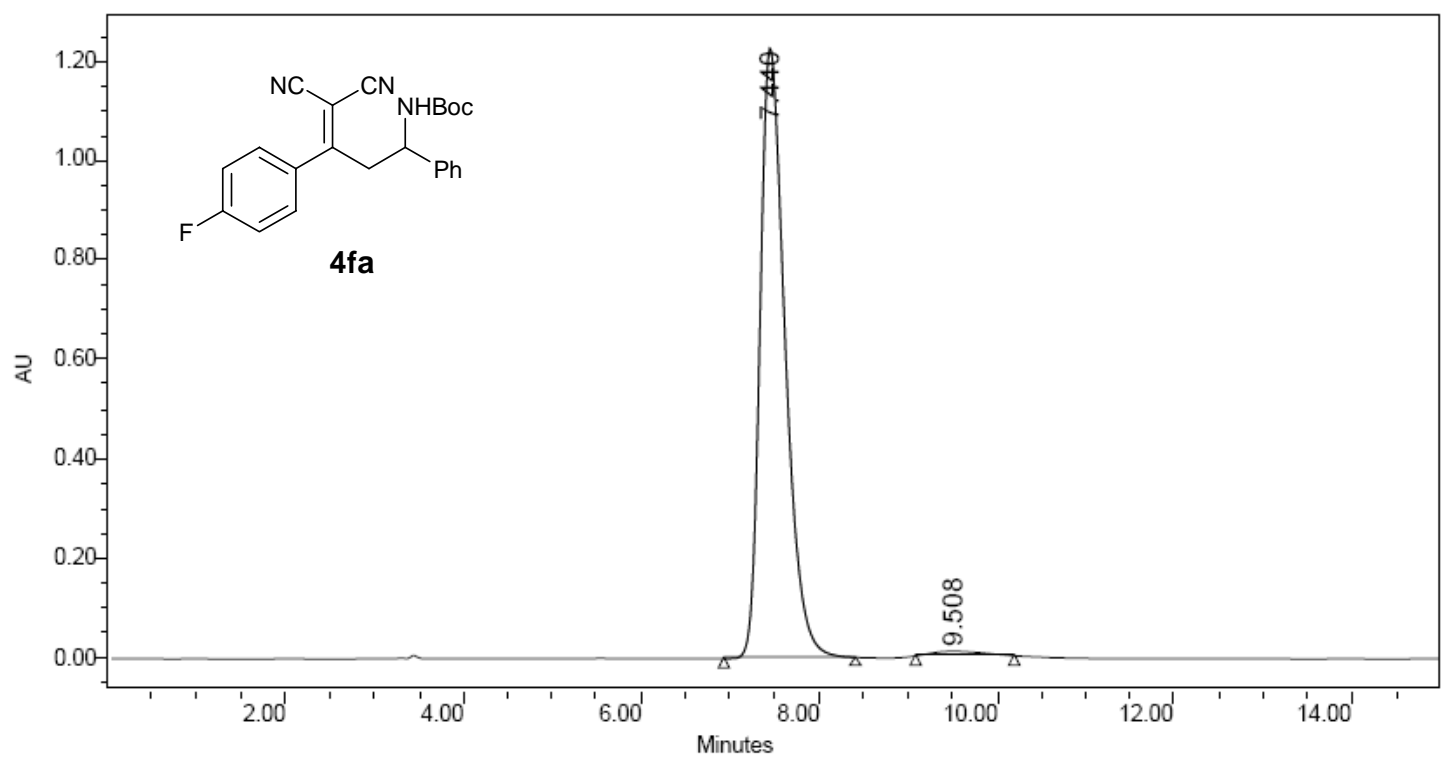

\begin{tabular}{|l|c|r|r|r|r|}
\hline & $\begin{array}{c}\mathrm{RT} \\
(\mathrm{min})\end{array}$ & $\begin{array}{c}\text { Area } \\
\left(\mathrm{V}{ }^{*} \mathrm{sec}\right)\end{array}$ & $\%$ Area & $\begin{array}{c}\text { Height } \\
(\mathrm{V})\end{array}$ & $\begin{array}{c}\% \\
\text { Height }\end{array}$ \\
\hline 1 & 7.440 & 23993911 & 99.37 & 1227835 & 99.58 \\
\hline 2 & 9.508 & 152274 & 0.63 & 5239 & 0.42 \\
\hline
\end{tabular}



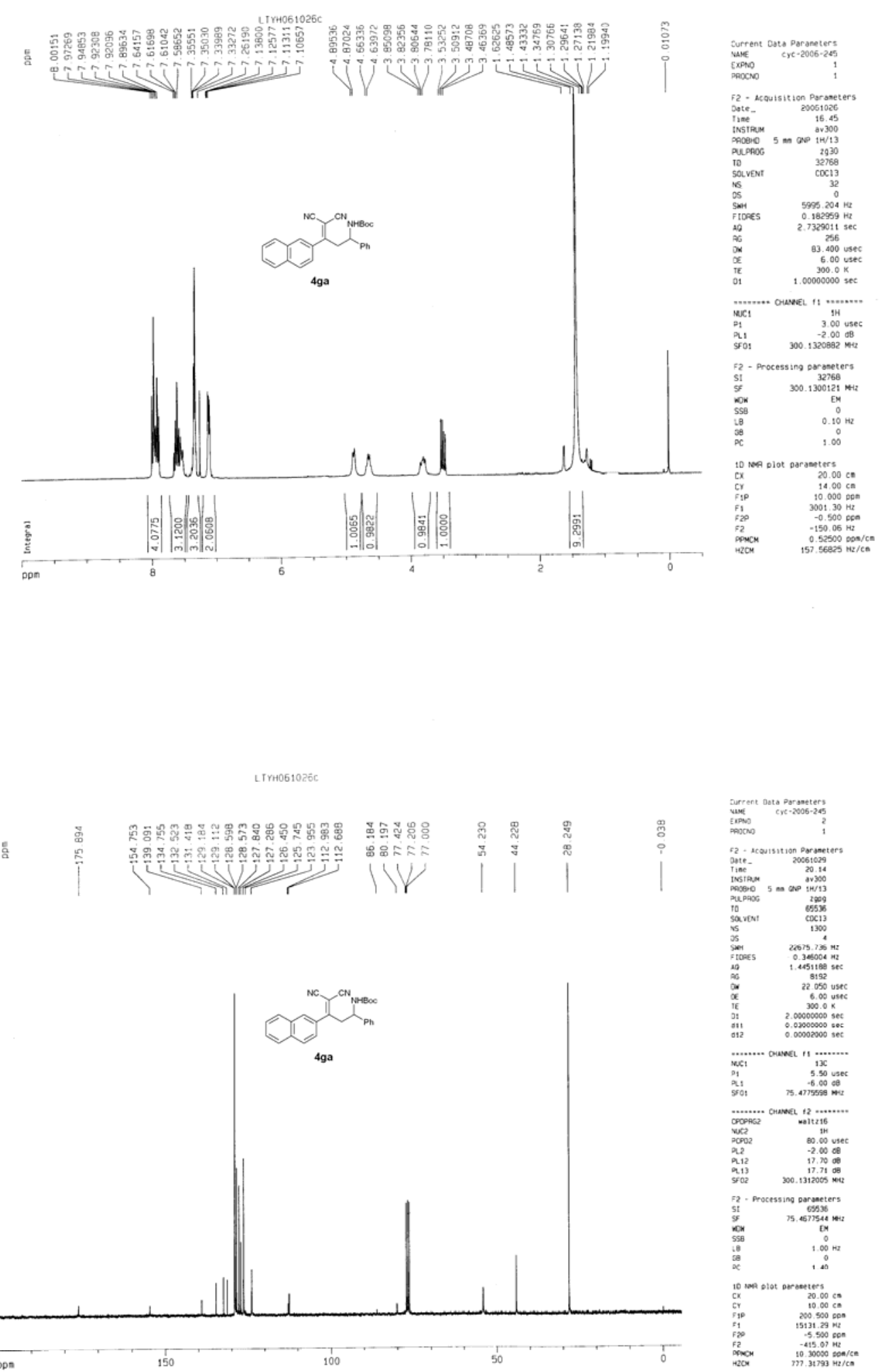
Mass Spectrum Molecular Formular Report

$\begin{array}{llll}\text { Analysis Info } & & \text { Acquisition Date } & \text { 10/30/2006 4:55:28 PM } \\ \text { Analysis Name } & \text { D:LBrukerldatalmsdatal1006111.d } & & \\ \text { Method } & \text { 1pass_pos_low.tofpar } & \text { Operator } & \text { operator name } \\ \text { Sample Name } & 10061 & \text { Instrument } & \text { BioTOF Q } \\ \text { Comment } & \text { ESI source } & & \end{array}$

Comment ESI source

Acquisition Parameter

$\begin{array}{llllll}\text { Capilary End Plate } & -4500 \mathrm{~V} & \text { Capilary Exit } & 120 \mathrm{~V} & \text { detbias } & 2 \mathrm{~V} \\ \text { EndP } & -4000 \mathrm{~V} & \text { Collision energy } & 0 \mathrm{eV} & \text { Number of } & 100\end{array}$

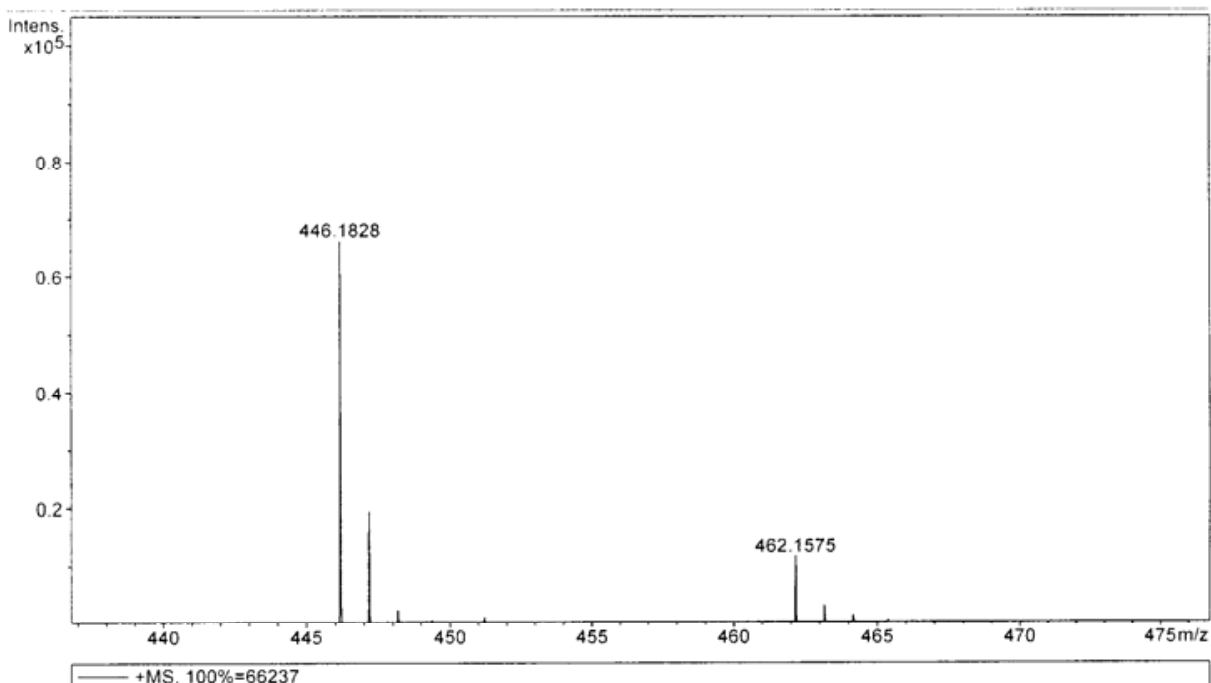

Sum Formula Sigma $\mathrm{m} / \mathrm{z} \quad$ Err [ppm] Mean Err [ppm] rdb N Rule $e^{-}$

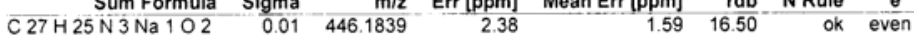

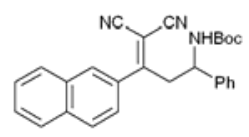

$4 \mathrm{ga}$ 

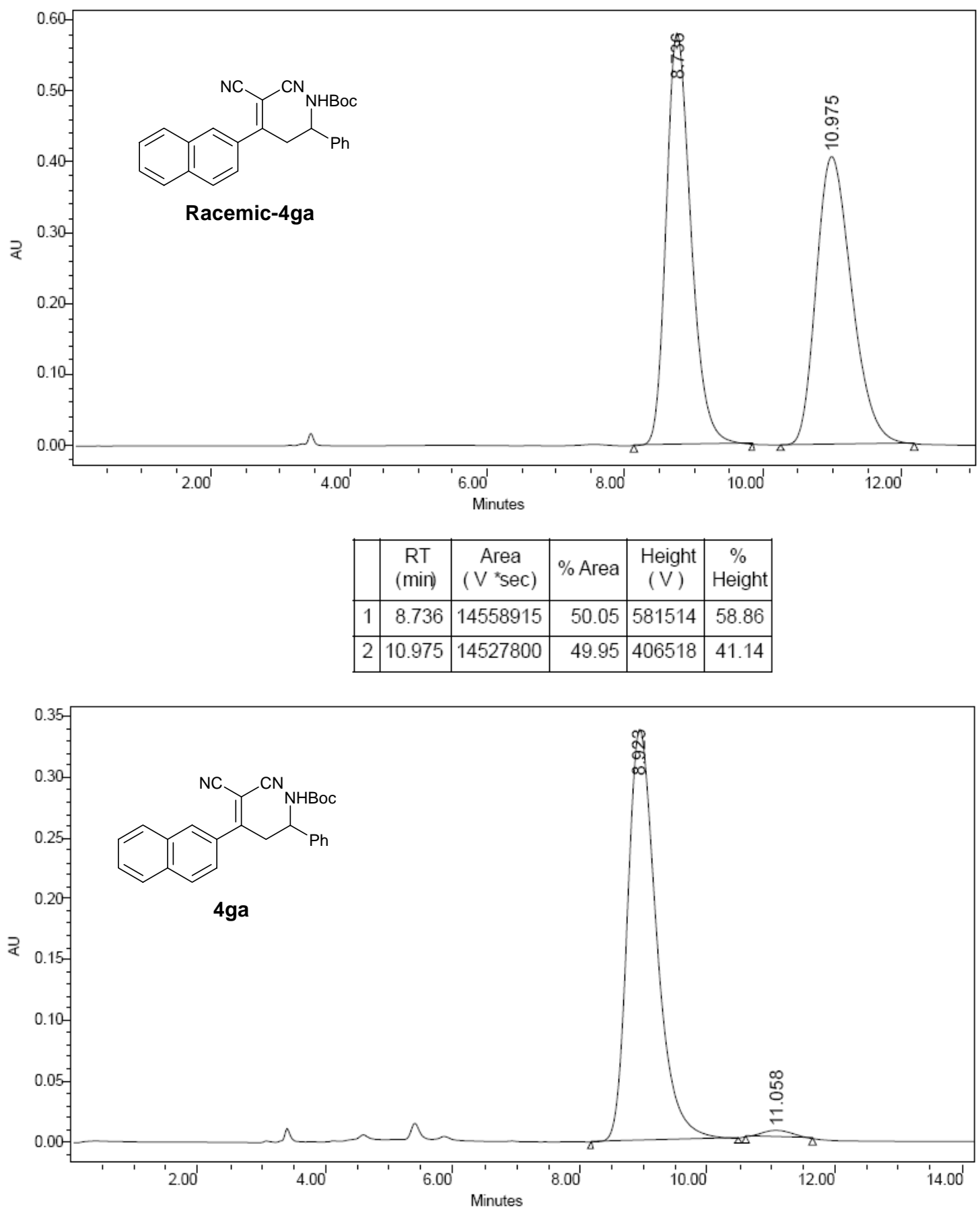

\begin{tabular}{|l|c|r|r|r|r|}
\hline & $\begin{array}{c}\mathrm{RT} \\
(\mathrm{min})\end{array}$ & $\begin{array}{c}\text { Area } \\
\left(\mathrm{V}{ }^{*} \mathrm{sec}\right)\end{array}$ & $\%$ Area & $\begin{array}{c}\text { Height } \\
(\mathrm{V})\end{array}$ & $\begin{array}{c}\% \\
\text { Height }\end{array}$ \\
\hline 1 & 8.923 & 10675947 & 99.30 & 338684 & 99.02 \\
\hline 2 & 11.058 & 75100 & 0.70 & 3338 & 0.98 \\
\hline
\end{tabular}



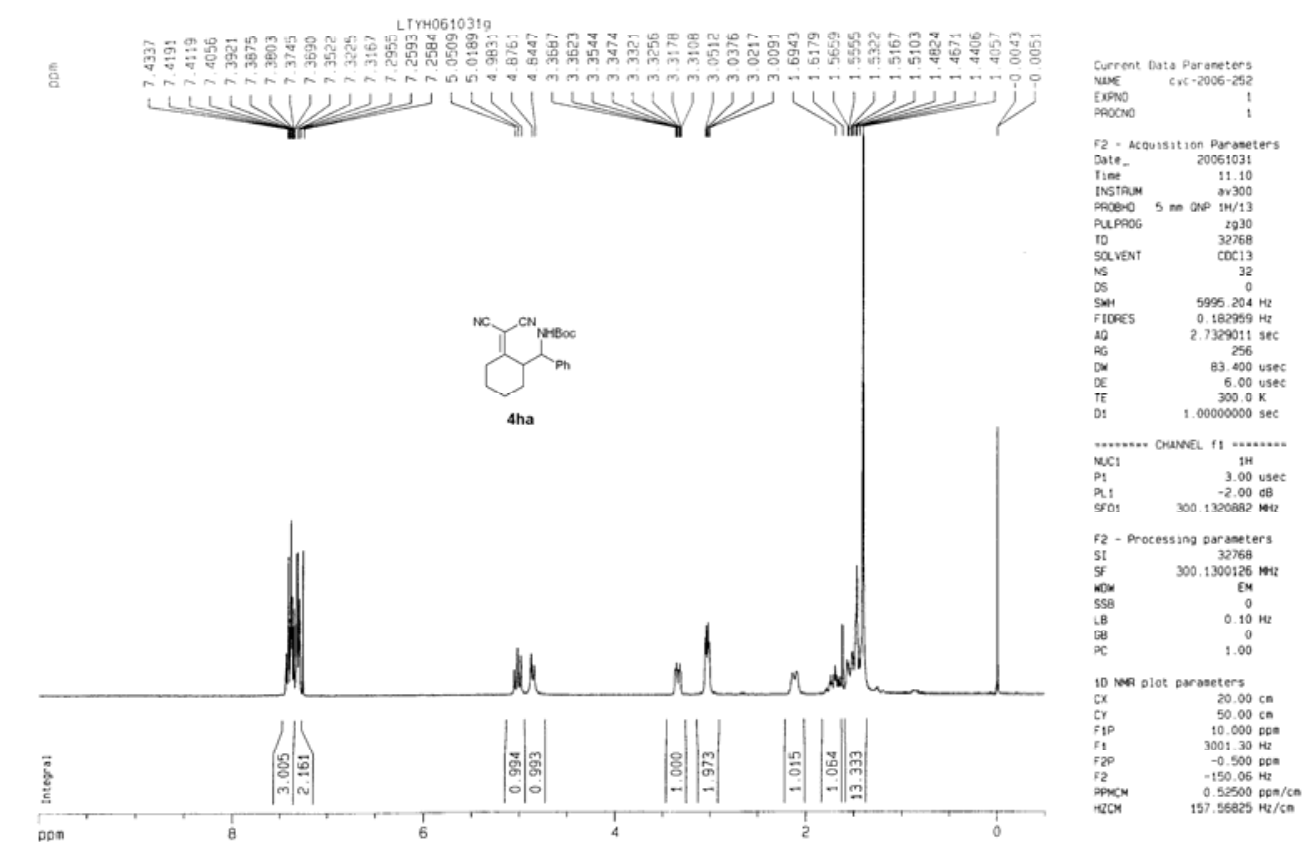

LIrHo6:0319

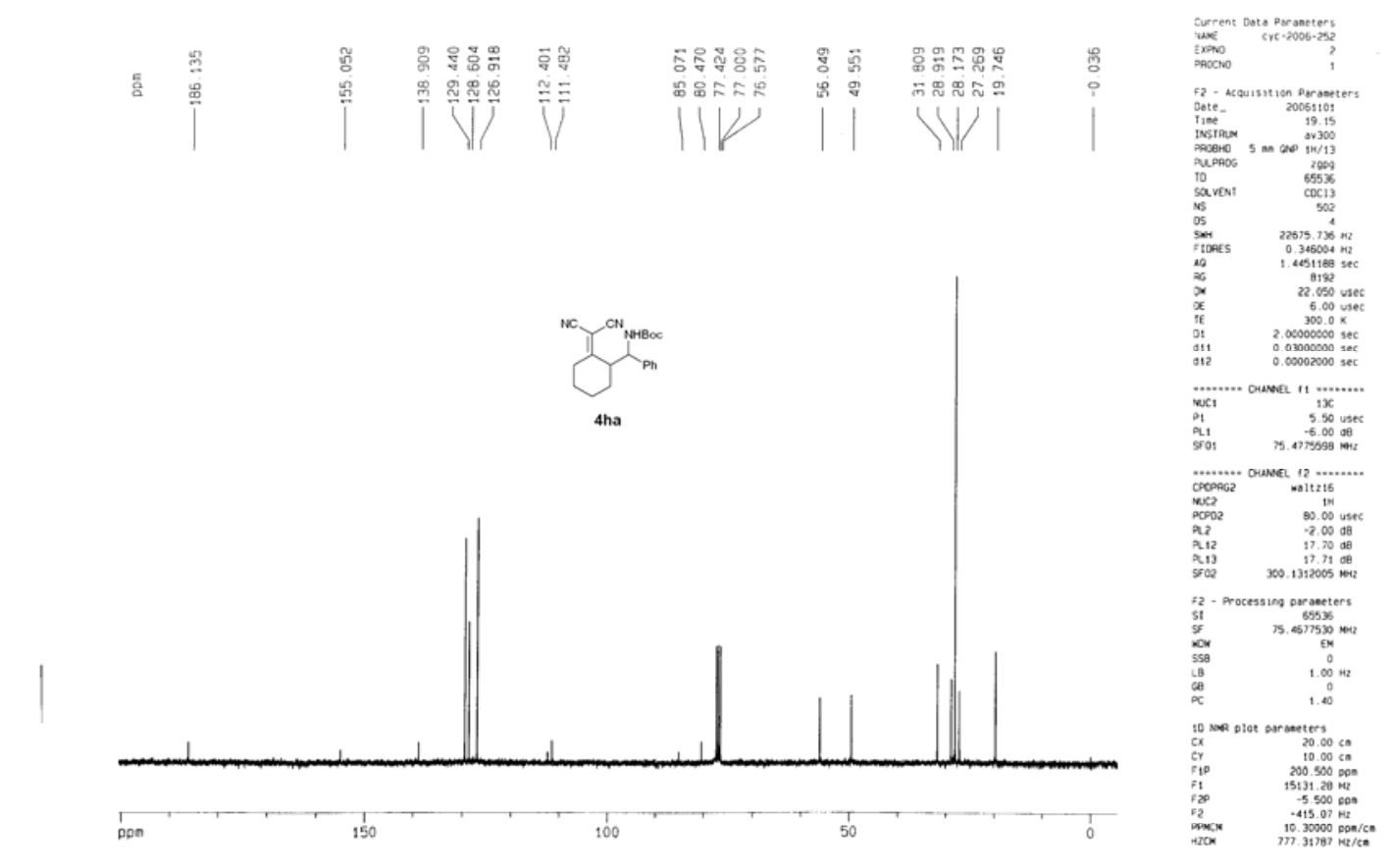


Mass Spectrum Molecular Formular Report

Analysis Info

Analysis Name D:IBrukerldatalmsdatal1007311.d

Method

Sample Name

Comment

Acquisition Parameter

Capilary End Plate .4500

EndP
Capilary Exit

Collision energy $120 \mathrm{~V}$

detbias

Number of

Averages

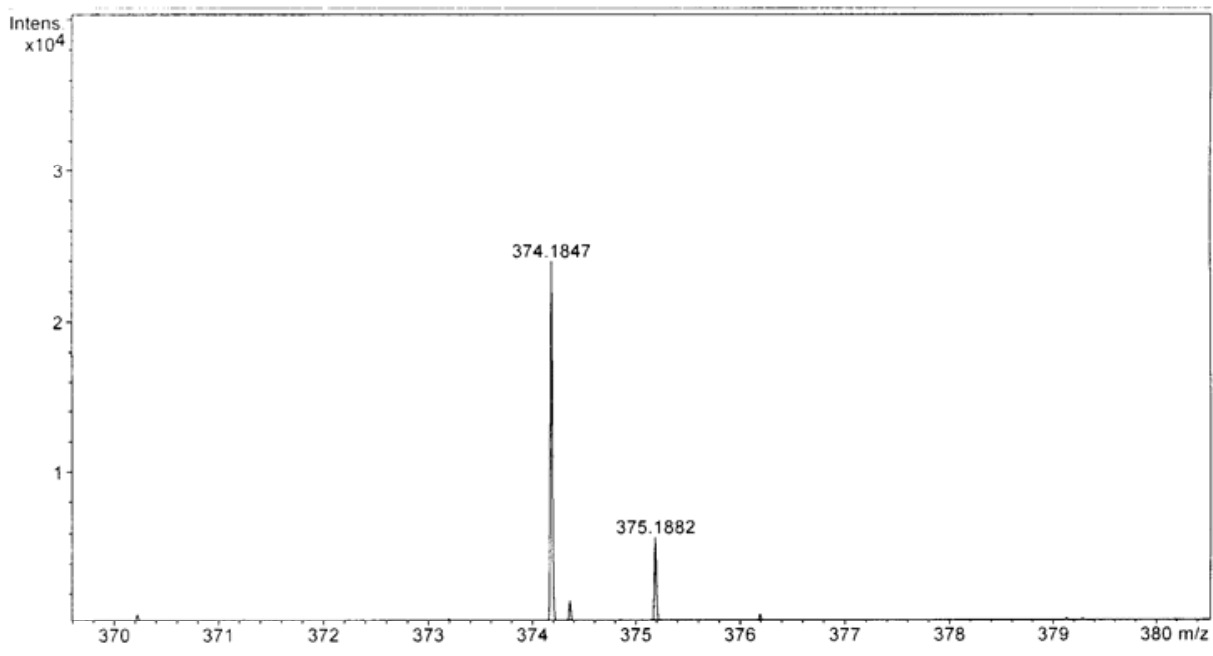

$-+M S, 100 \%=34641$

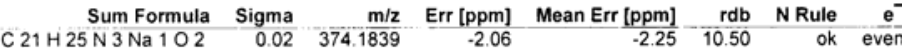

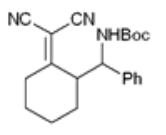

4ha 


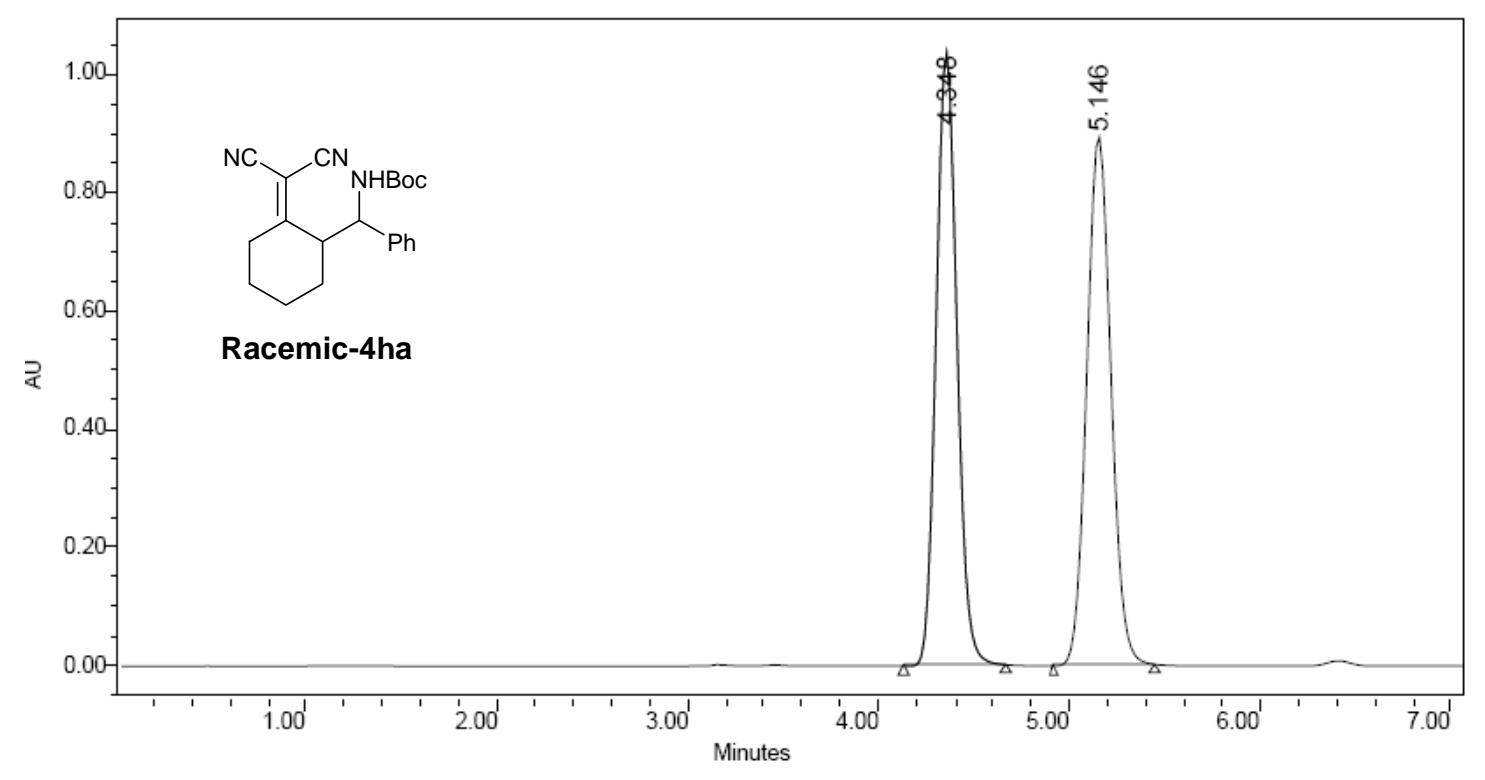

\begin{tabular}{|c|c|c|c|c|c|}
\hline & $\begin{array}{c}\mathrm{RT} \\
(\mathrm{min})\end{array}$ & $\begin{array}{c}\text { Area } \\
\left(\mathrm{V}^{*} \mathrm{sec}\right)\end{array}$ & $\%$ Area & $\begin{array}{c}\text { Height } \\
(\mathrm{V})\end{array}$ & $\begin{array}{c}\% \\
\text { Height }\end{array}$ \\
\hline 1 & 4.348 & 7894511 & 49.96 & 1037277 & 53.65 \\
\hline 2 & 5.146 & 7905826 & 50.04 & 896233 & 46.35 \\
\hline
\end{tabular}

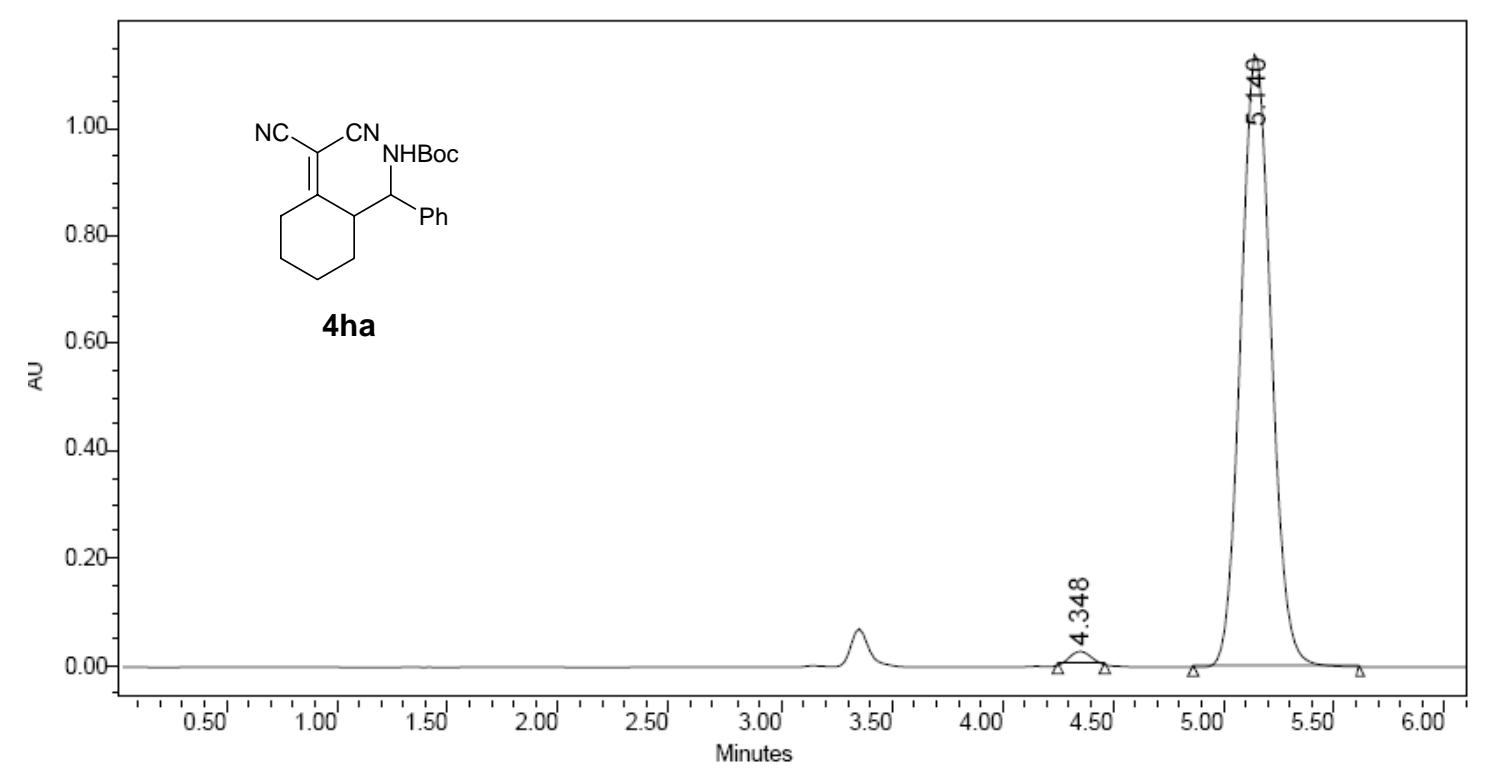

\begin{tabular}{|l|c|r|r|r|r|}
\hline & $\begin{array}{c}\mathrm{RT} \\
(\mathrm{min})\end{array}$ & $\begin{array}{c}\text { Area } \\
\left(\mathrm{V}{ }^{*} \mathrm{sec}\right)\end{array}$ & $\%$ Area & $\begin{array}{c}\text { Height } \\
(\mathrm{V})\end{array}$ & $\begin{array}{c}\% \\
\text { Height }\end{array}$ \\
\hline 1 & 4.348 & 80888 & 0.74 & 16664 & 1.44 \\
\hline 2 & 5.140 & 10880844 & 99.26 & 1143788 & 98.56 \\
\hline
\end{tabular}



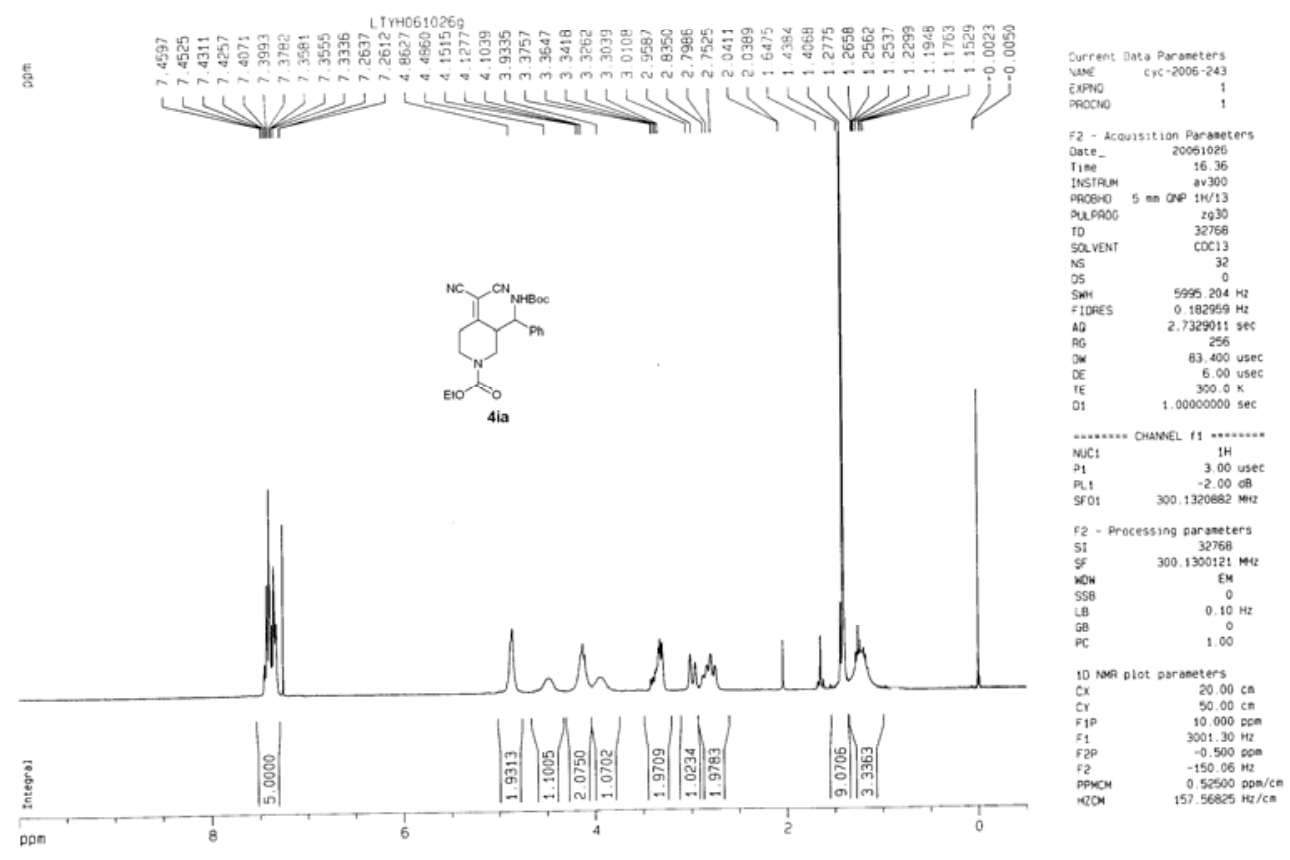

LTYHC610269

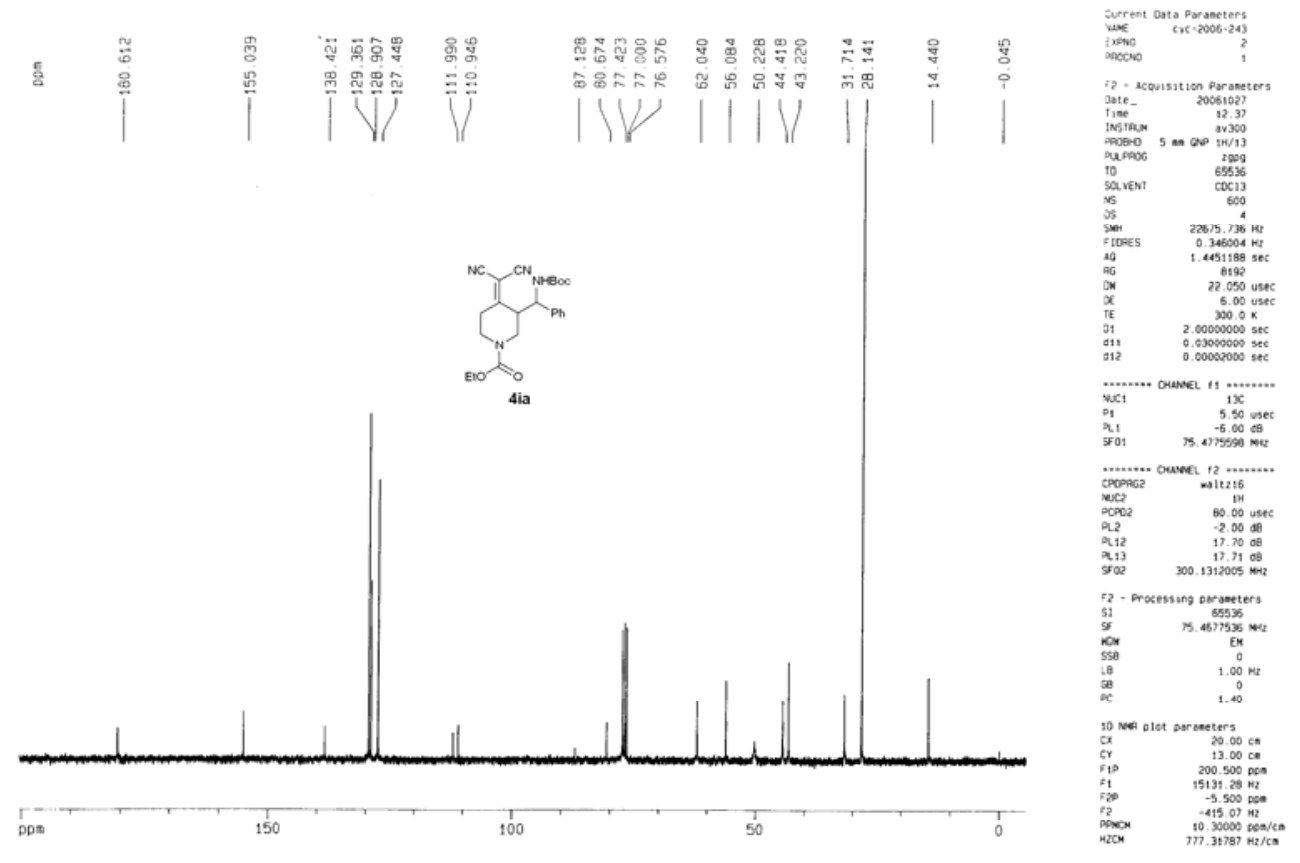


Mass Spectrum Molecular Formular Report

Analysis Info

Analysis Name D:IBrukerldatalmsdatal1007011.d

Method

Sample Name

Comment

Acquisition Parameter

Capilary End Plate -4500

$\begin{array}{ll}\text { Capilary End Plate } & -4500 \\ \text { EndP } & -4000 \mathrm{~V}\end{array}$

$\begin{array}{lll} & \text { Capilary Exit } & 120 \mathrm{~V} \\ \text { Collision energy } & 0 \mathrm{eV}\end{array}$
Acquisition Date 10/30/2006 4:59:08 PM

Operator

Instrument

or name

BioTOF Q

Int

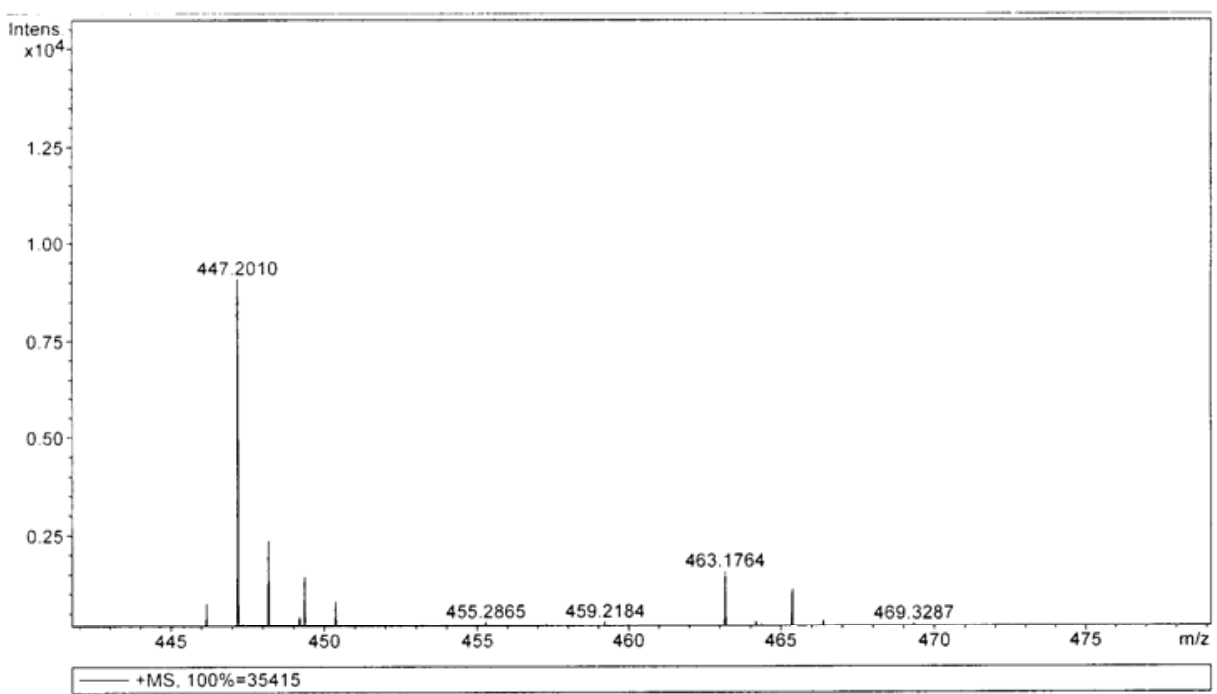

Sum Formula Sigma $\mathrm{m} / \mathbf{z}$ Err [ppm] Mean Err [ppm] rdb NRule $e^{-}$

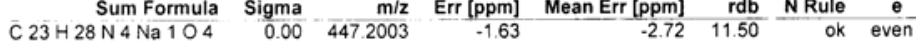

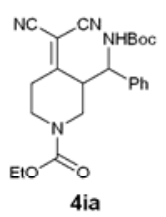




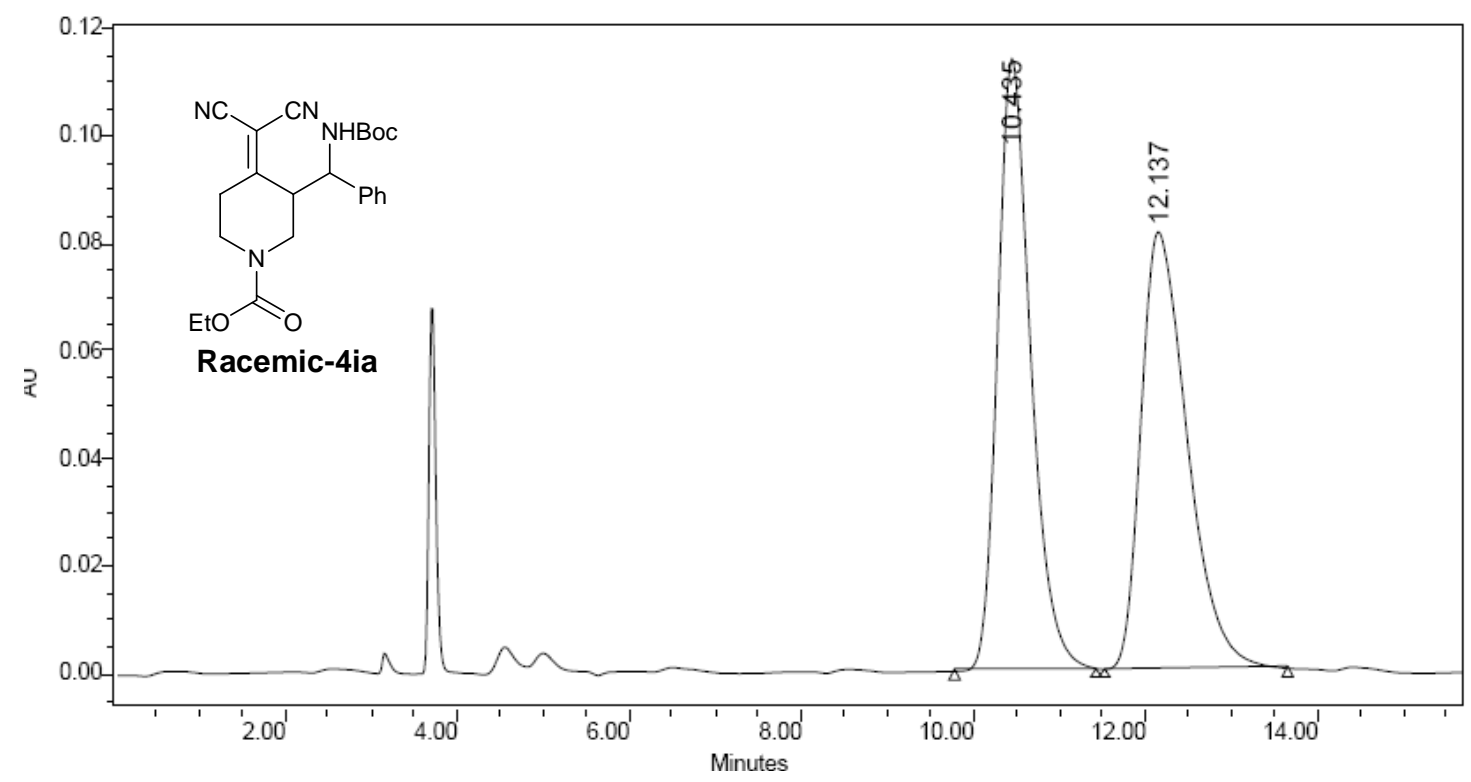

\begin{tabular}{|c|c|c|c|c|c|}
\hline & $\begin{array}{c}\text { RT } \\
(\mathrm{min})\end{array}$ & $\begin{array}{c}\text { Area } \\
(\mathrm{V} \text { *sec })\end{array}$ & $\%$ Area & $\begin{array}{c}\text { Height } \\
(\mathrm{V})\end{array}$ & $\begin{array}{c}\% \\
\text { Height }\end{array}$ \\
\hline 1 & 10.435 & 3044789 & 50.08 & 113598 & 58.32 \\
\hline 2 & 12.137 & 3035071 & 49.92 & 81194 & 41.68 \\
\hline
\end{tabular}

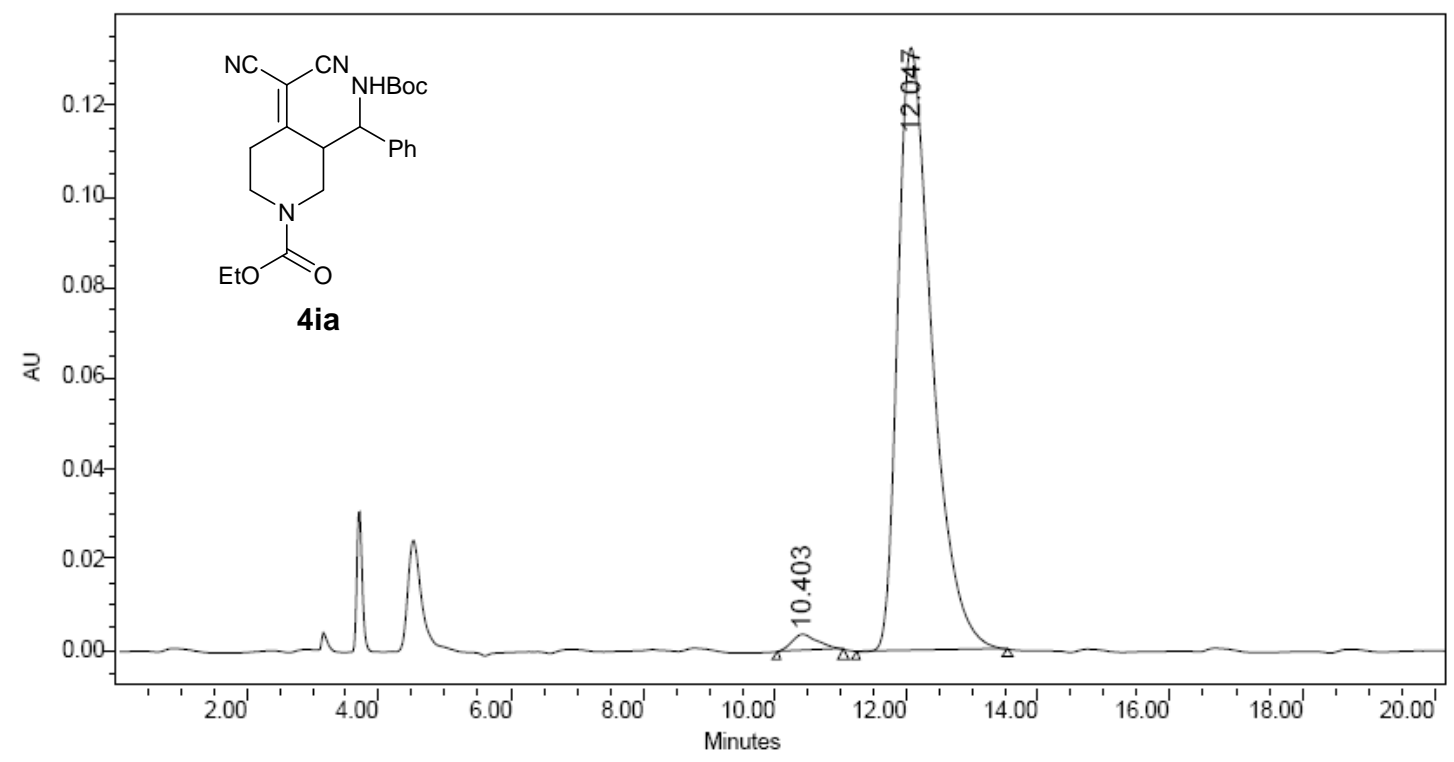

\begin{tabular}{|c|c|r|r|r|r|}
\hline & $\begin{array}{c}\text { RT } \\
(\mathrm{min})\end{array}$ & $\begin{array}{c}\text { Area } \\
\left(\mathrm{V}^{*} \mathrm{sec}\right)\end{array}$ & $\%$ Area & $\begin{array}{c}\text { Height } \\
(\mathrm{V})\end{array}$ & $\begin{array}{c}\% \\
\text { Height }\end{array}$ \\
\hline 1 & 10.403 & 56436 & 1.16 & 2715 & 2.00 \\
\hline 2 & 12.047 & 4801306 & 98.84 & 132995 & 98.00 \\
\hline
\end{tabular}




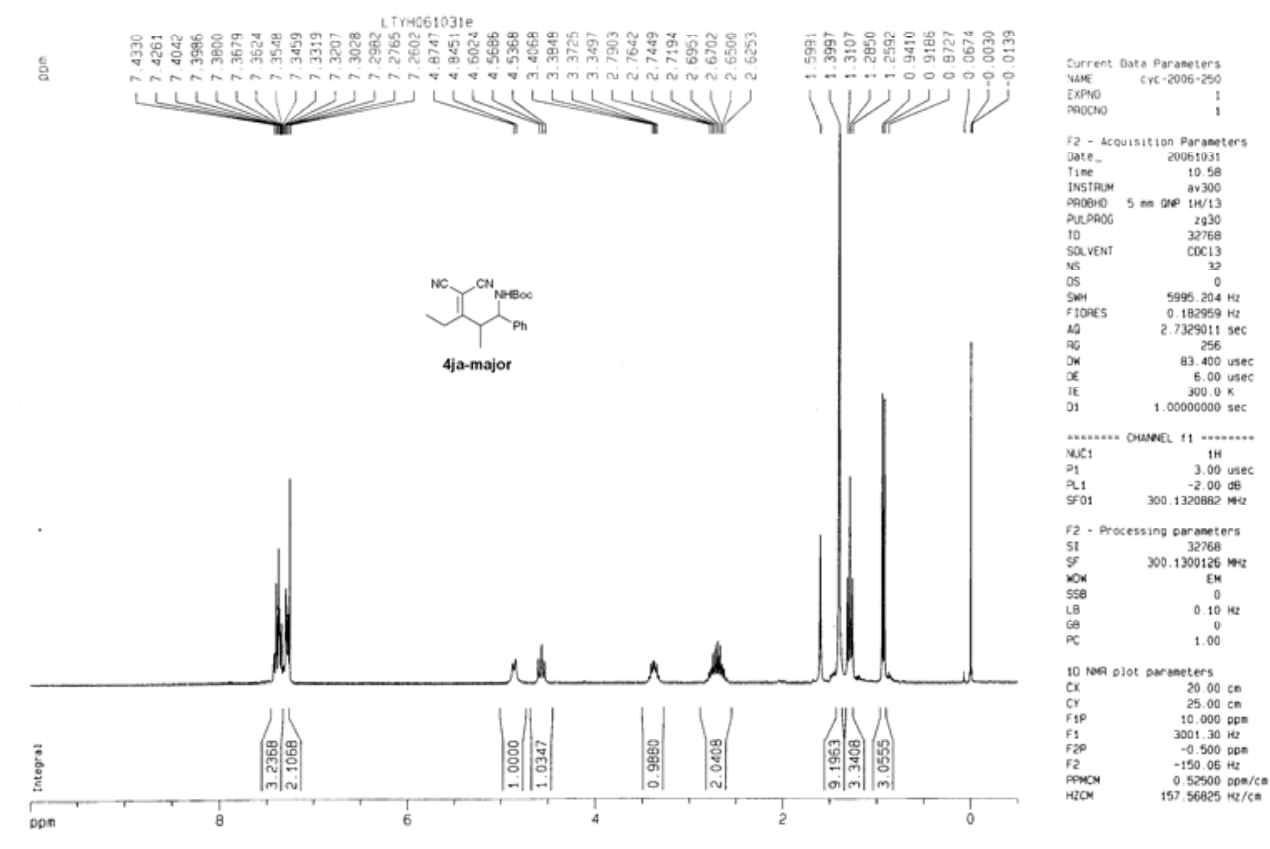

LIrH061031e

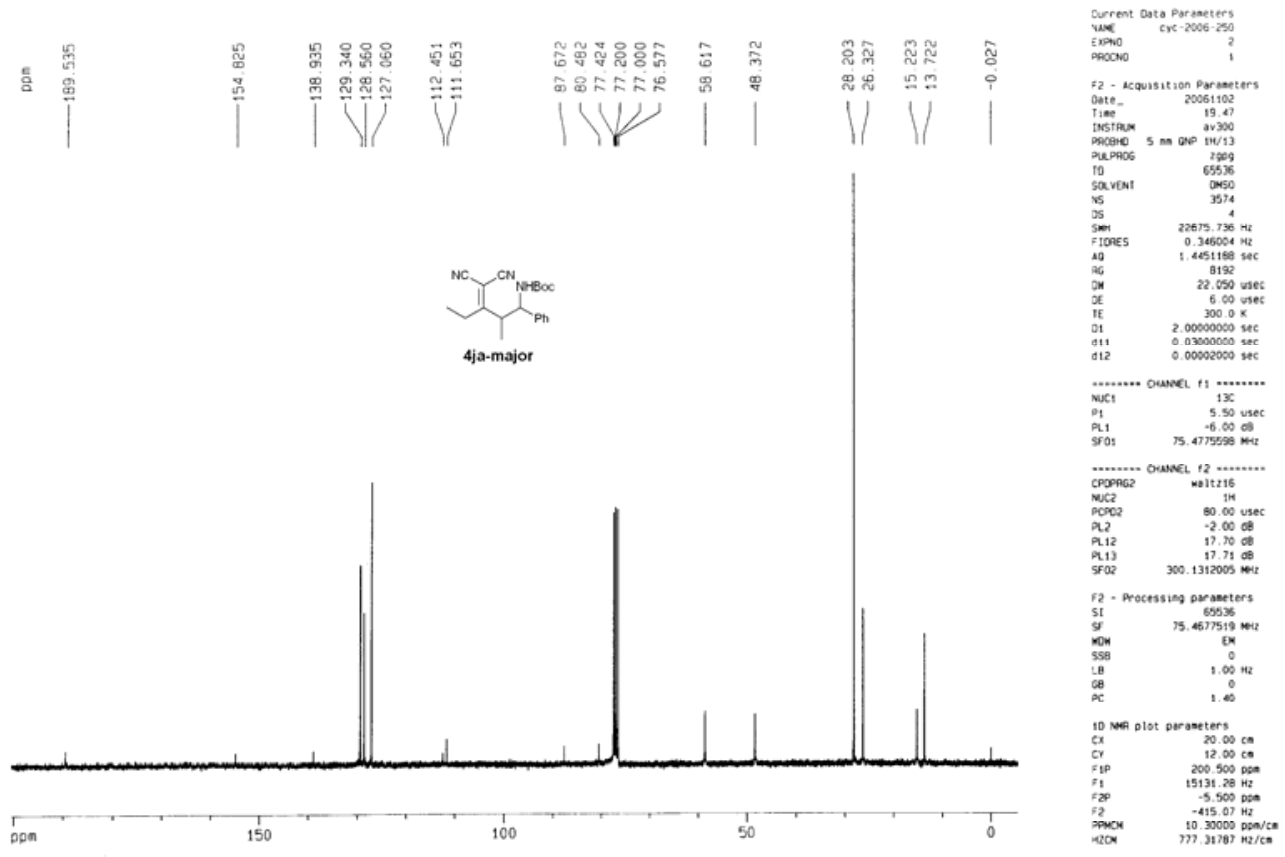




\section{Mass Spectrum Molecular Formular Report}

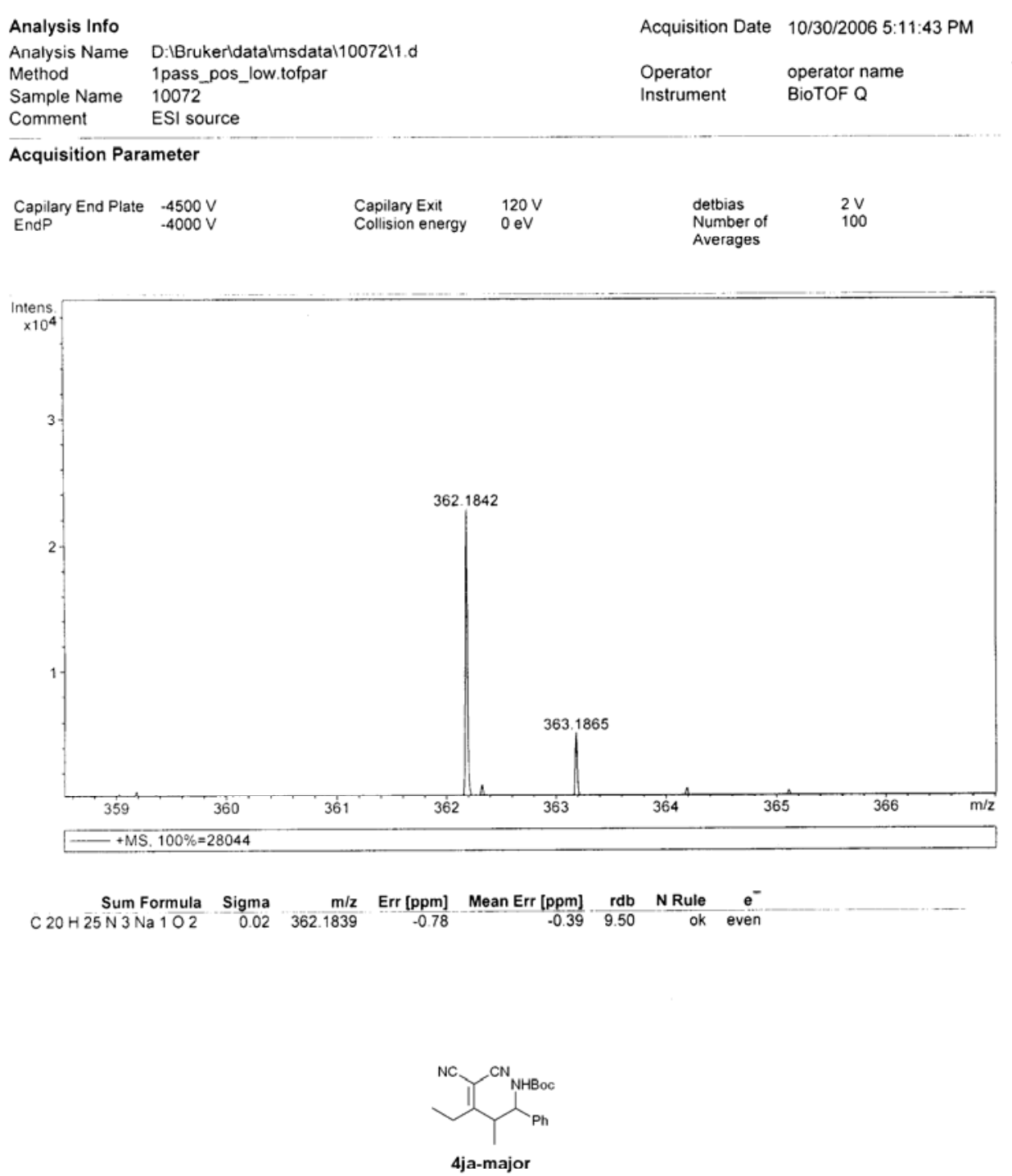

Bruker Daltonics DátaAnalysis 3.2

printed: $\quad 10 \overline{30} / 2006 \quad 05: 12: 48 \mathrm{PM}$

Page 1 of 1 

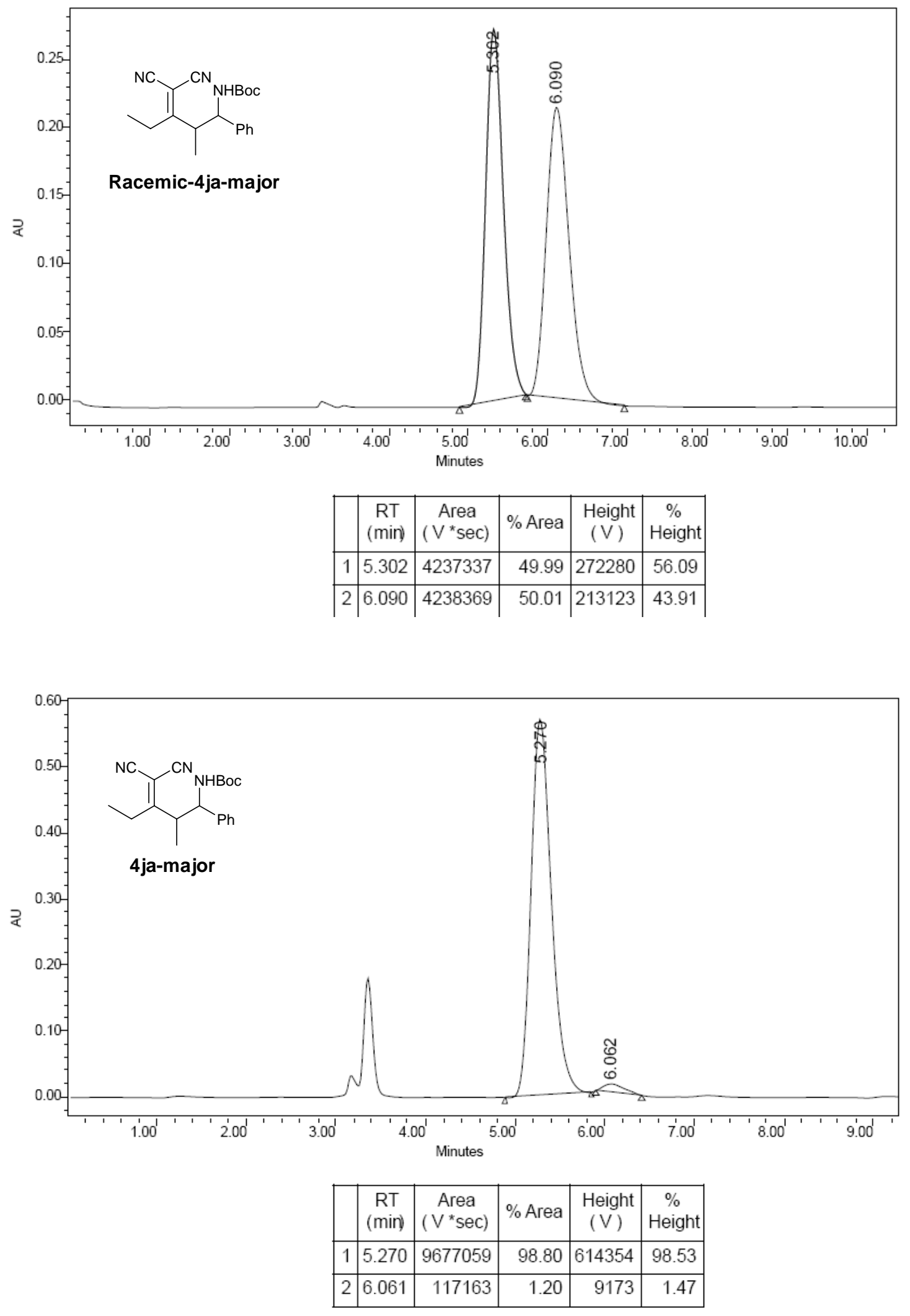


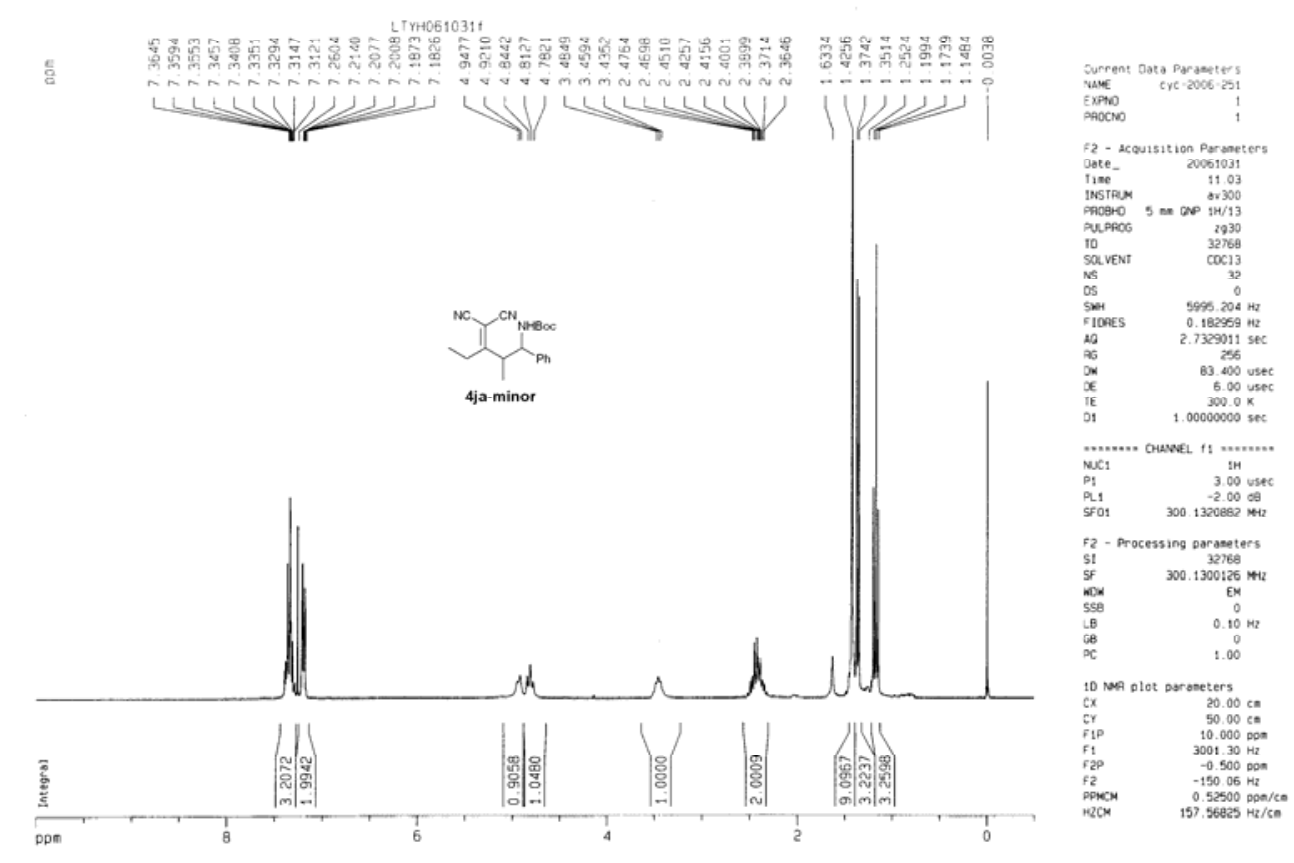

Lirmeto 103:

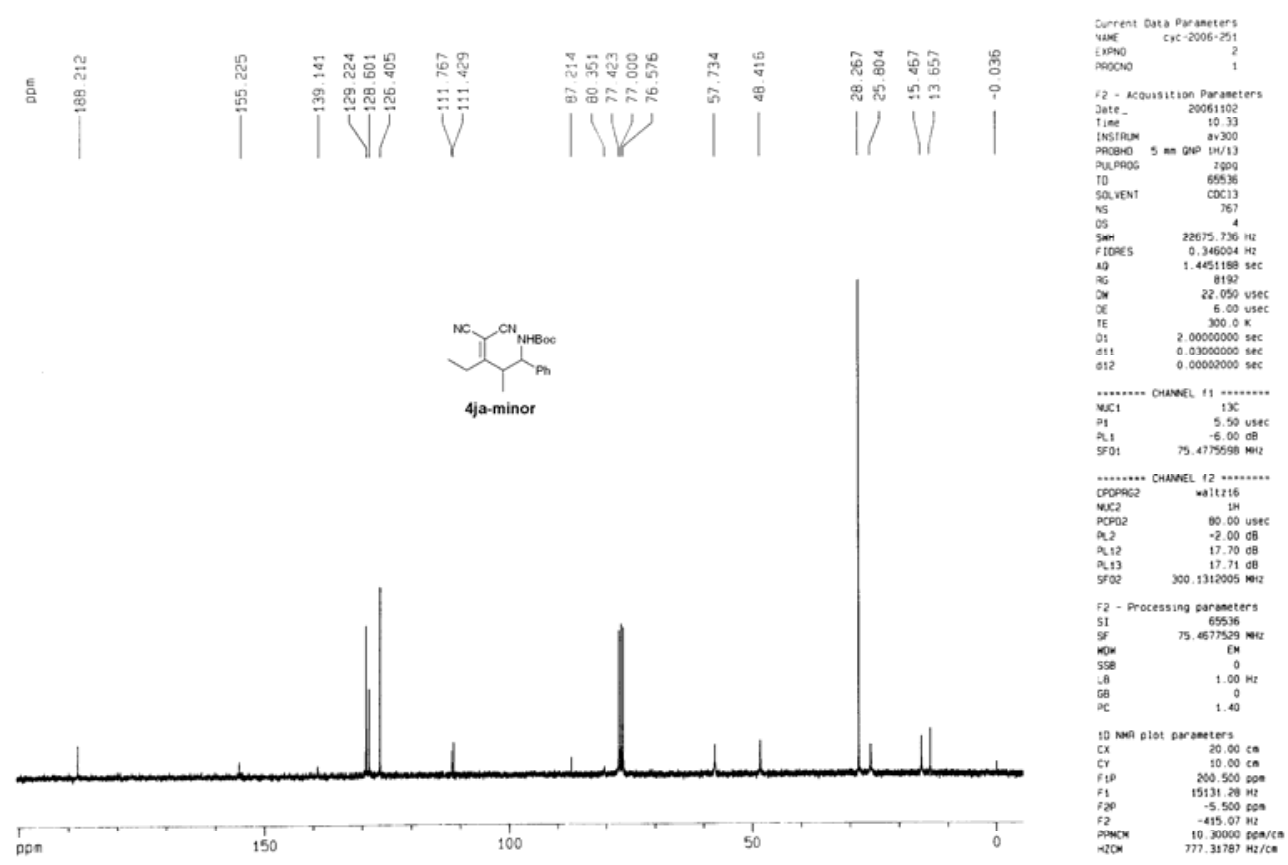




\section{Mass Spectrum Molecular Formular Report}

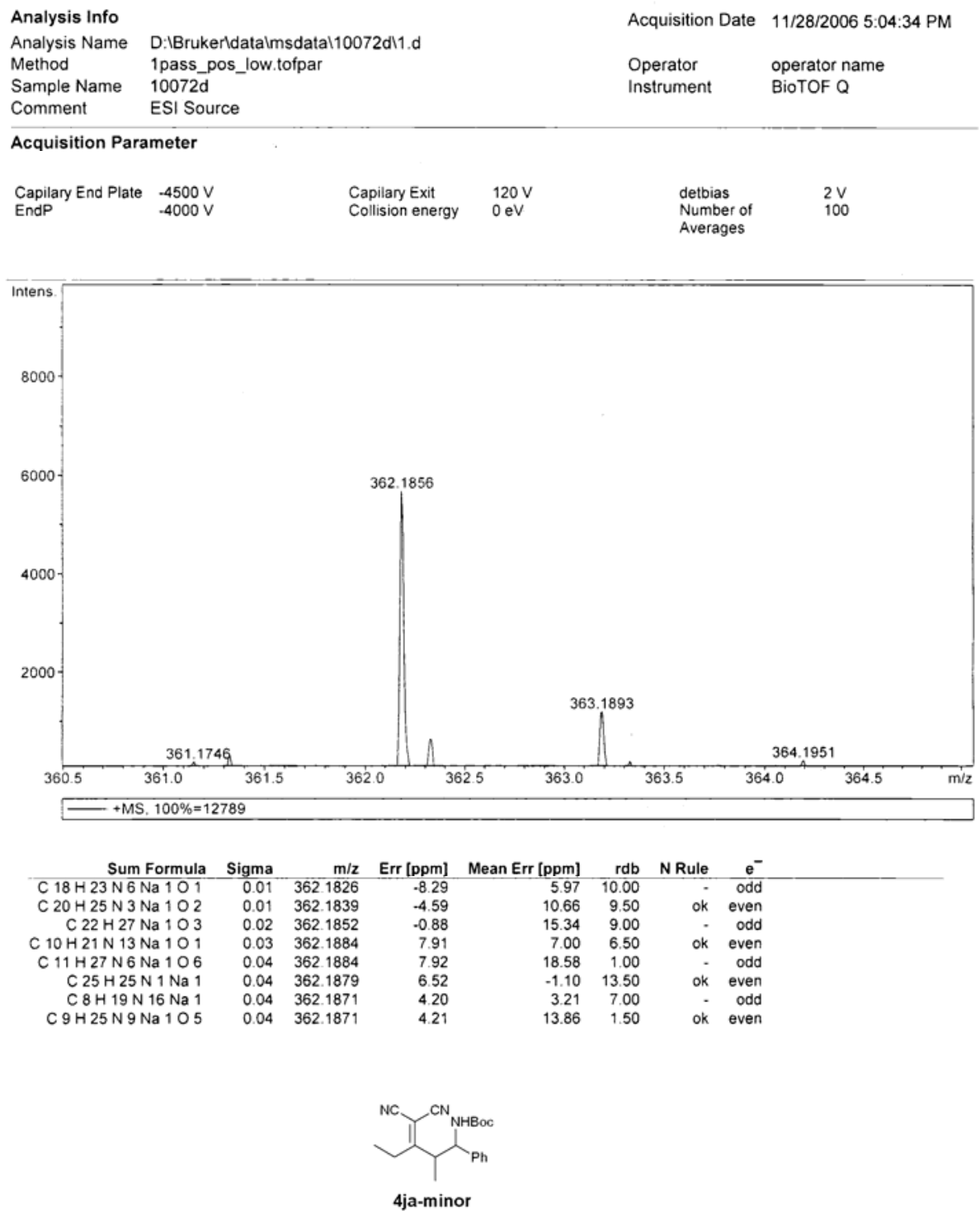

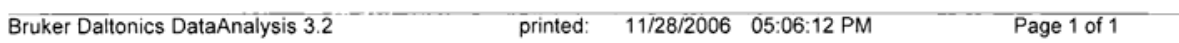




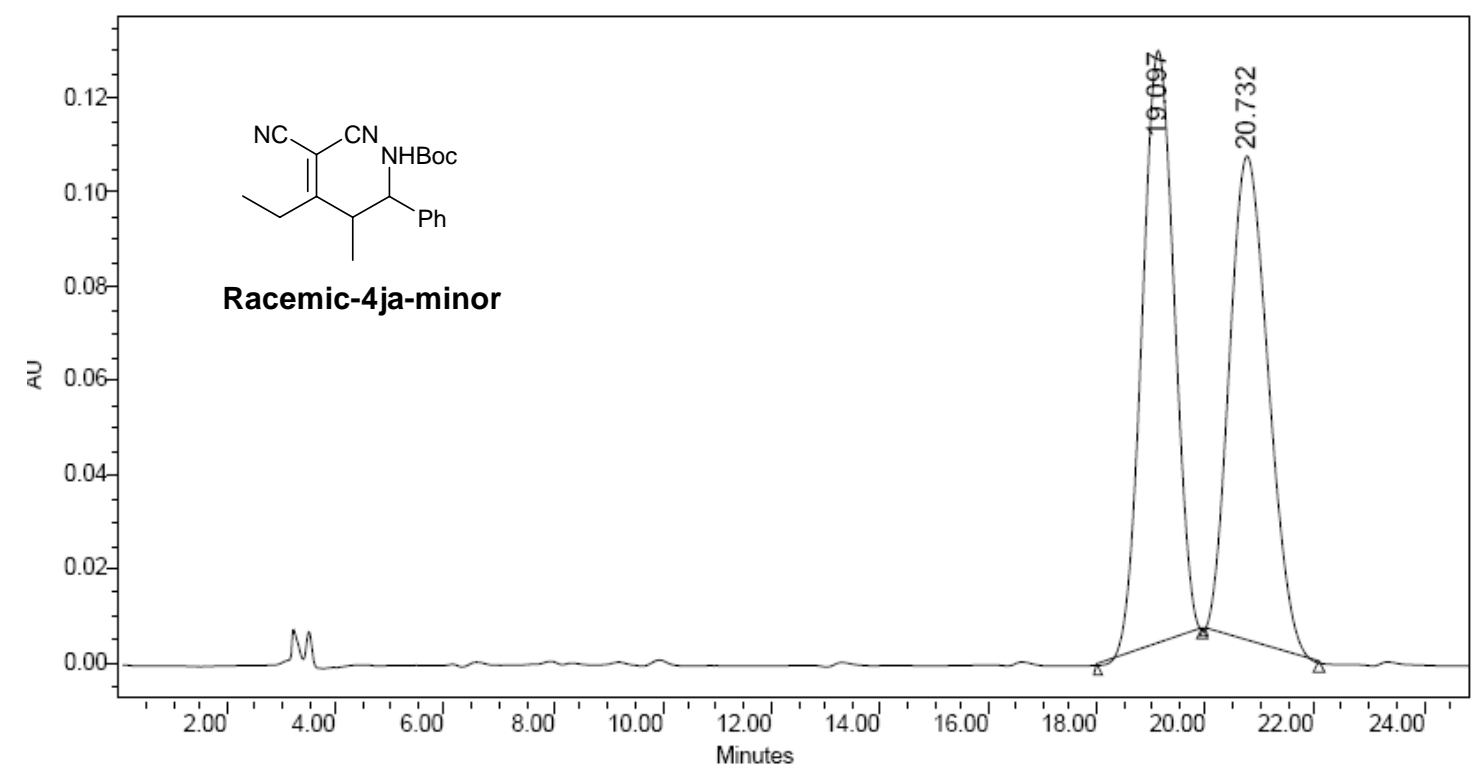

\begin{tabular}{|c|c|c|c|c|c|}
\hline & $\begin{array}{c}\text { RT } \\
(\mathrm{min})\end{array}$ & $\begin{array}{c}\text { Area } \\
\left(\mathrm{V}^{*} \mathrm{sec}\right)\end{array}$ & $\%$ Area & $\begin{array}{c}\text { Height } \\
(\mathrm{V})\end{array}$ & $\begin{array}{c}\% \\
\text { Height }\end{array}$ \\
\hline 1 & 19.097 & 5173205 & 50.40 & 126367 & 55.09 \\
\hline 2 & 20.732 & 5091956 & 49.60 & 103003 & 44.91 \\
\hline
\end{tabular}

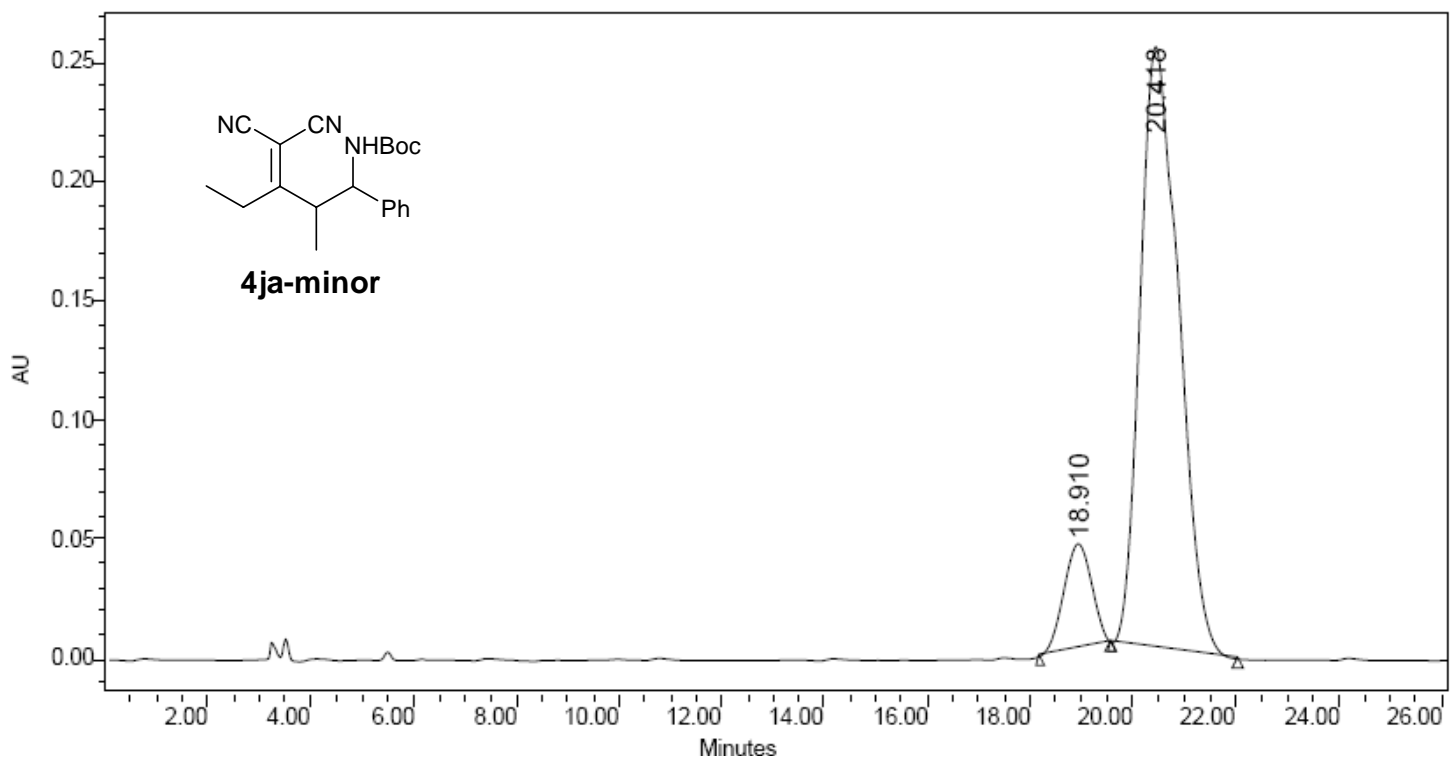

\begin{tabular}{|c|c|c|c|c|c|}
\hline & $\begin{array}{c}\mathrm{RT} \\
(\mathrm{min})\end{array}$ & $\begin{array}{c}\text { Area } \\
(\mathrm{V} \text { *sec })\end{array}$ & $\%$ Area & $\begin{array}{c}\text { Height } \\
(\mathrm{V})\end{array}$ & $\begin{array}{c}\% \\
\text { Height }\end{array}$ \\
\hline 1 & 18.910 & 1676755 & 11.28 & 44000 & 14.85 \\
\hline 2 & 20.418 & 13183543 & 88.72 & 252256 & 85.15 \\
\hline
\end{tabular}



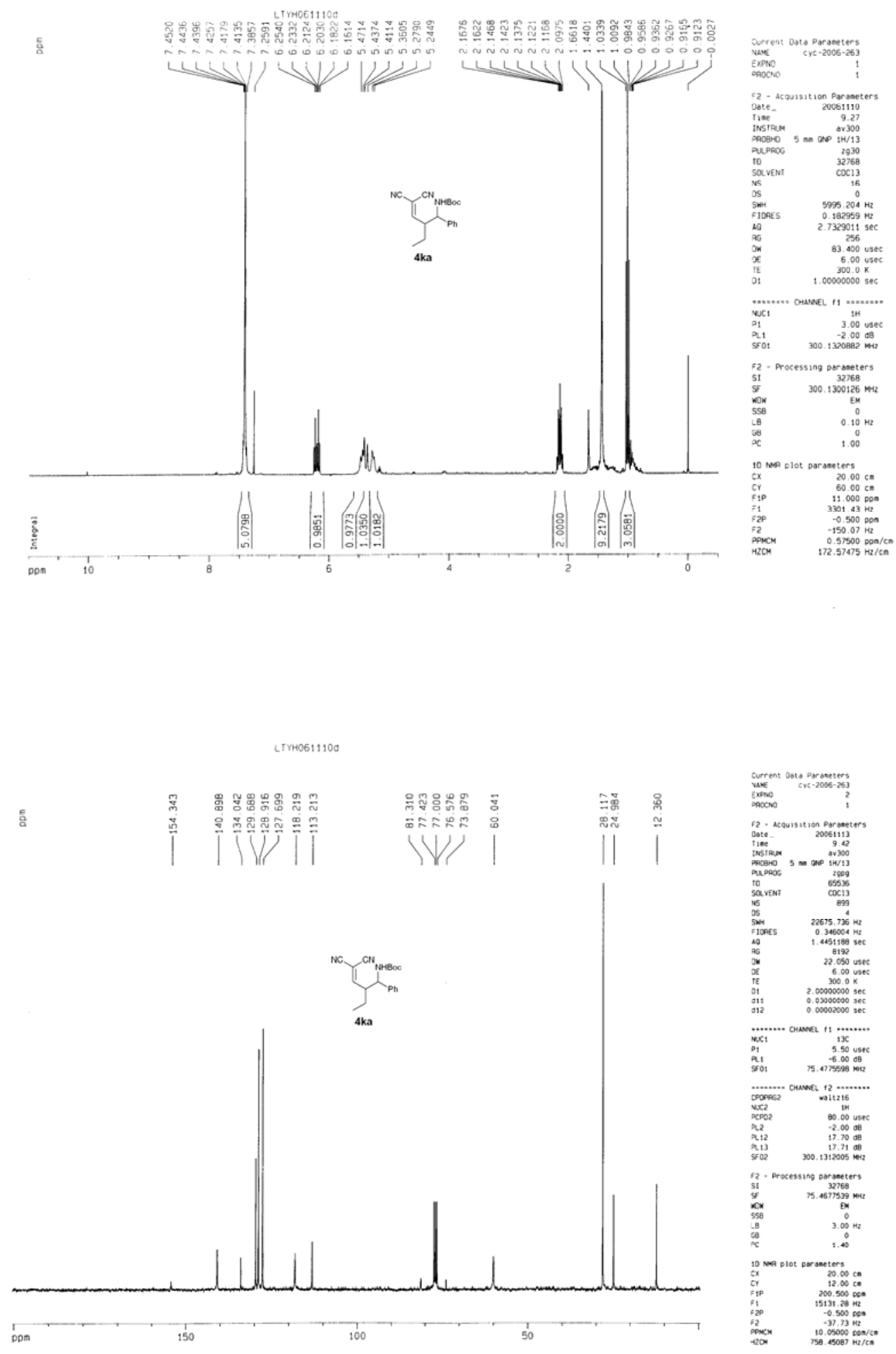


\section{Mass Spectrum Molecular Formular Report}

Analysis Info

Analysis Name D:IBrukerldatalmsdatal10097\1.d

Method

Sample Name

Comment

pass_pos low.tofpa

10097

ESI source

\section{Acquisition Paramete}

Capilary End Plate -4500 $-4000 \mathrm{~V}$
Capilary Exit $\quad 120 \mathrm{~V}$
Acquisition Date 11/6/2006 3:42:00 PM

Operator

Instrument

perator name

BioTOF Q

detbias

Number of

$2 \mathrm{~V}$

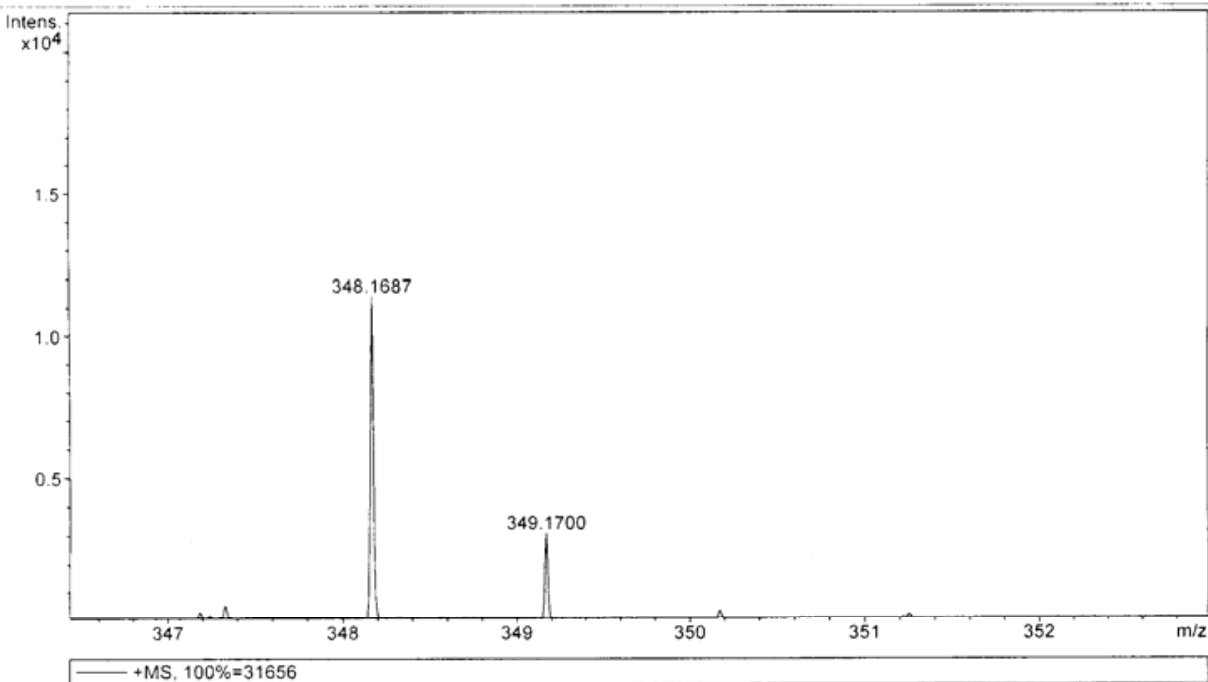

Sum Formula Sigma $\mathrm{m} / \mathrm{z}$ Err [ppm] Mean Err [ppm] rdb NRule e

C $19 \mathrm{H}_{2} 3 \mathrm{~N} 3 \mathrm{Na} 102 \quad 0.03 \quad 348.1682 \quad-1.16 \quad 0.14 \quad 9.50$ ok even<smiles>CCC(C=C(C#N)C#N)C(NC(=O)O)c1ccccc1</smiles>

$4 \mathrm{ka}$ 

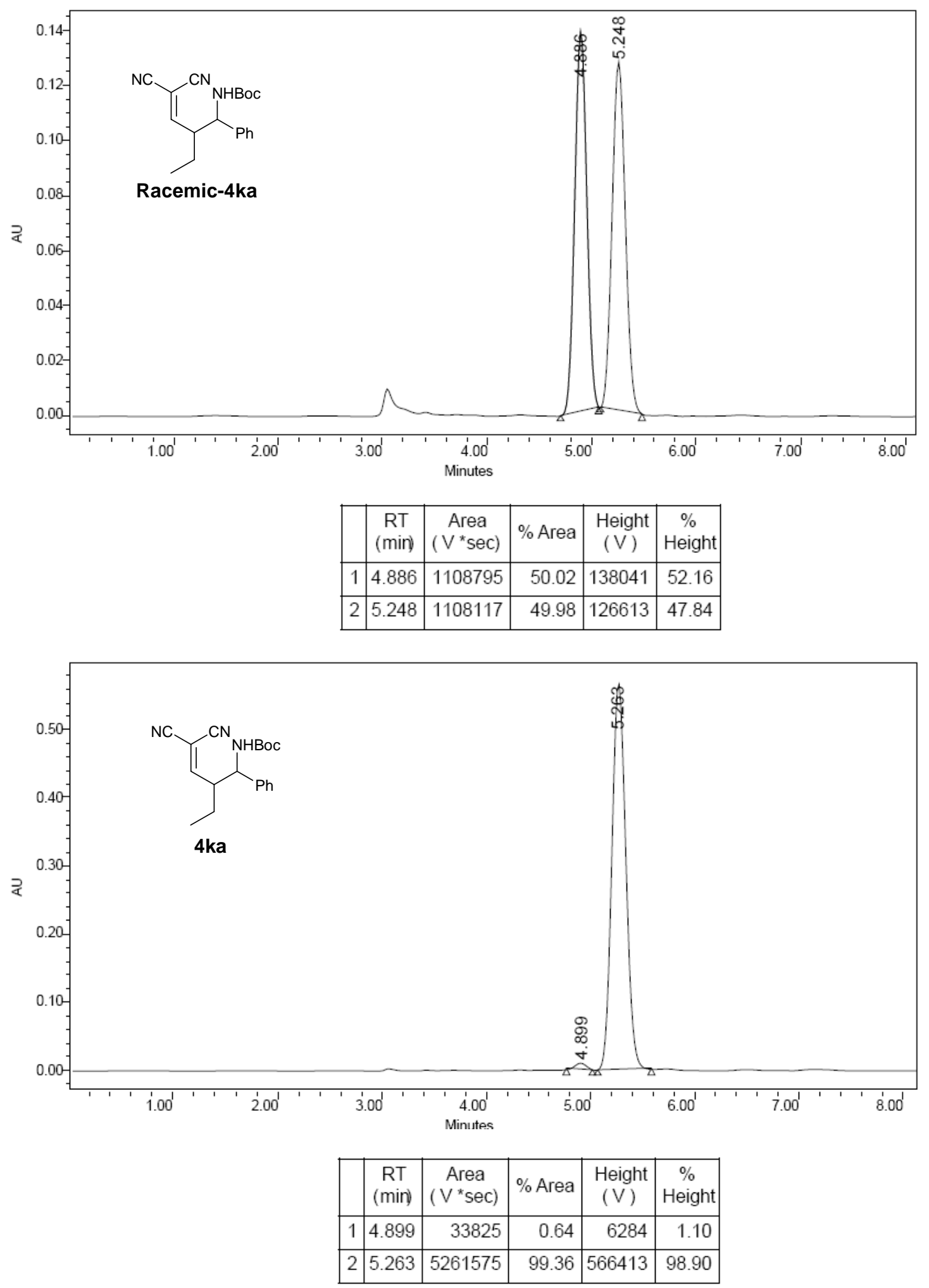

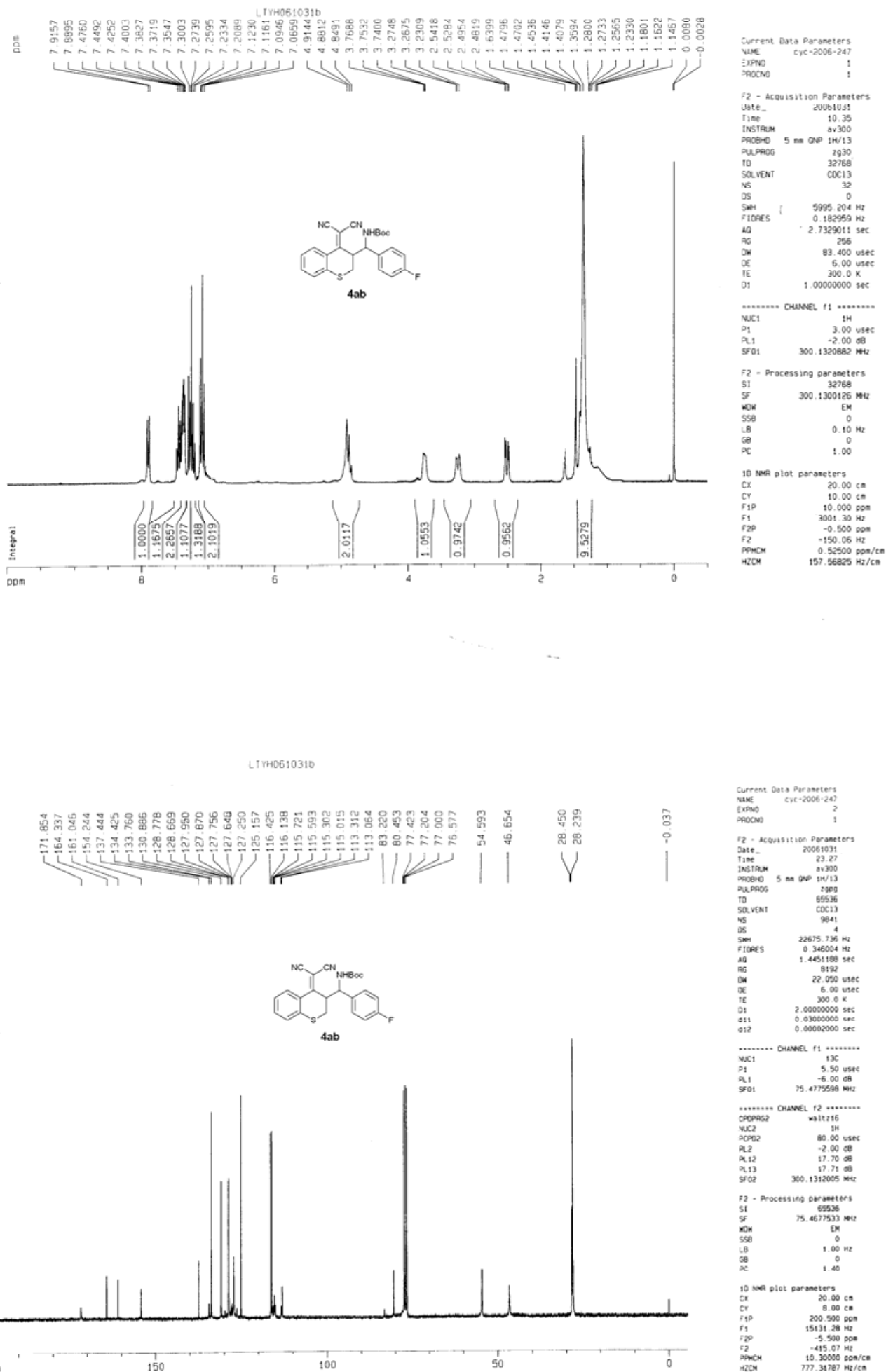


\section{Mass Spectrum Molecular Formular Report}

Analysis Info

Analysis Name D:IBrukerldatalmsdatal1007611.d

Method

Sample Name

Comment

pass pos low.tofpa

10076

ESI source

\section{Acquisition Parameter}

EndP

$-4000 \mathrm{~V}$ $\begin{array}{ll}\text { Capilary Exit } & 120 \mathrm{~V} \\ \text { Collision energy } & 0 \mathrm{eV}\end{array}$
Acquisition Date 10/30/2006 5:00:07 PM

Operator

Instrument

operator name

BioTOF Q

Number of $\quad 100$ Averages

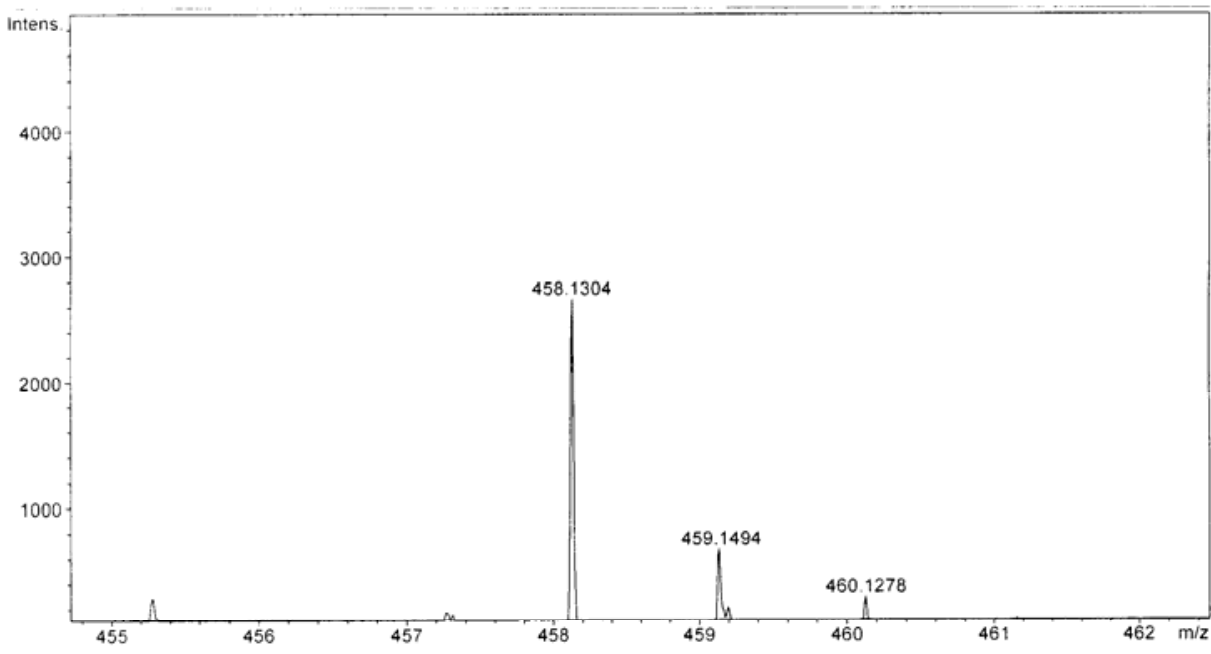

Sum Formula Sigma $\mathrm{m} / \mathrm{z}$ Err [ppm] Mean Err [ppm] rdb N Rule e-

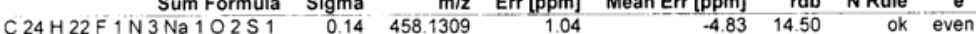

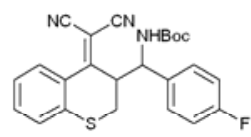

$4 a b$ 


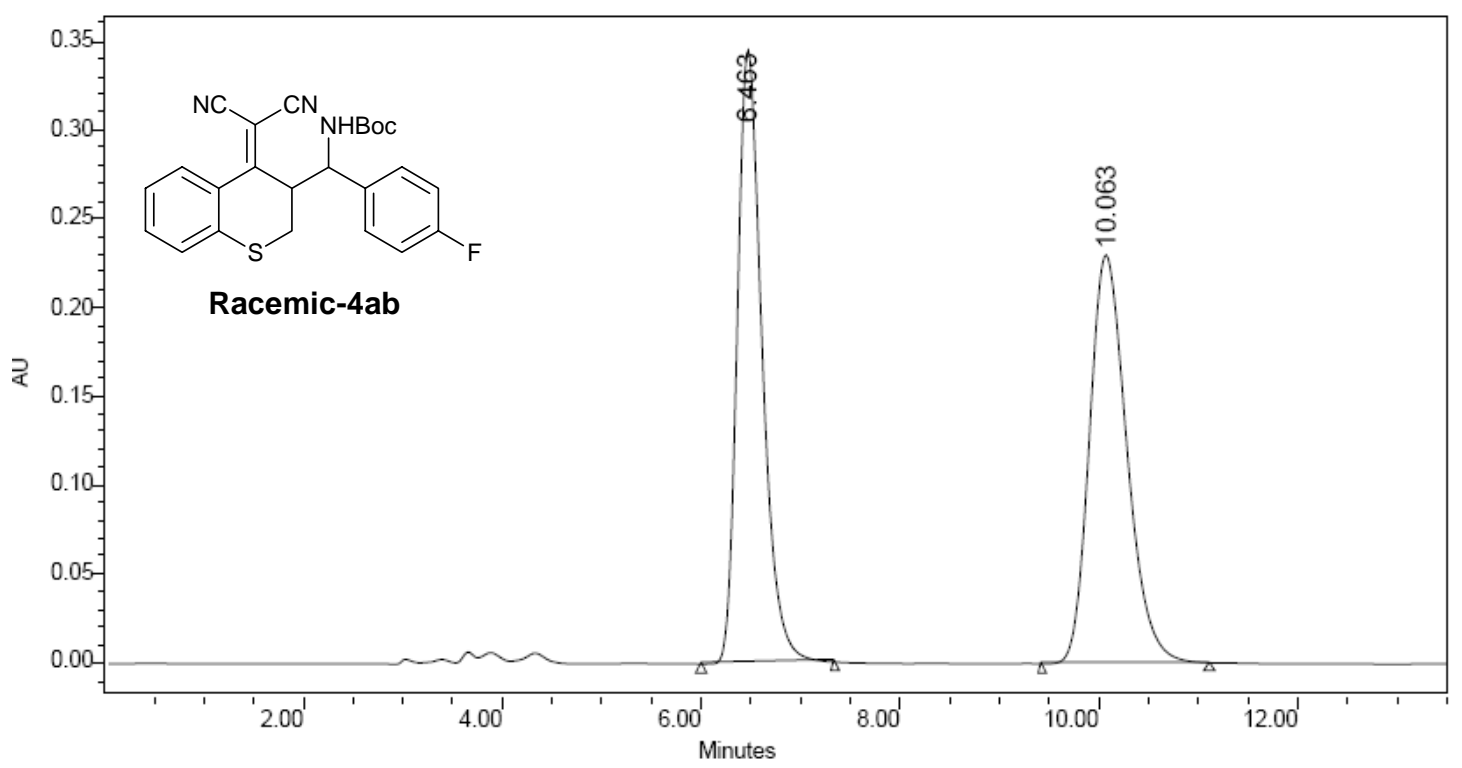

\begin{tabular}{|c|c|c|c|c|c|}
\hline & $\begin{array}{c}\text { RT } \\
(\mathrm{min})\end{array}$ & $\begin{array}{c}\text { Area } \\
\left(\mathrm{V}^{*} \mathrm{sec}\right)\end{array}$ & $\%$ Area & $\begin{array}{c}\text { Height } \\
(\mathrm{V})\end{array}$ & $\begin{array}{c}\% \\
\text { Height }\end{array}$ \\
\hline 1 & 6.463 & 6016791 & 50.03 & 345453 & 60.04 \\
\hline 2 & 10.063 & 6009395 & 49.97 & 229930 & 39.96 \\
\hline
\end{tabular}

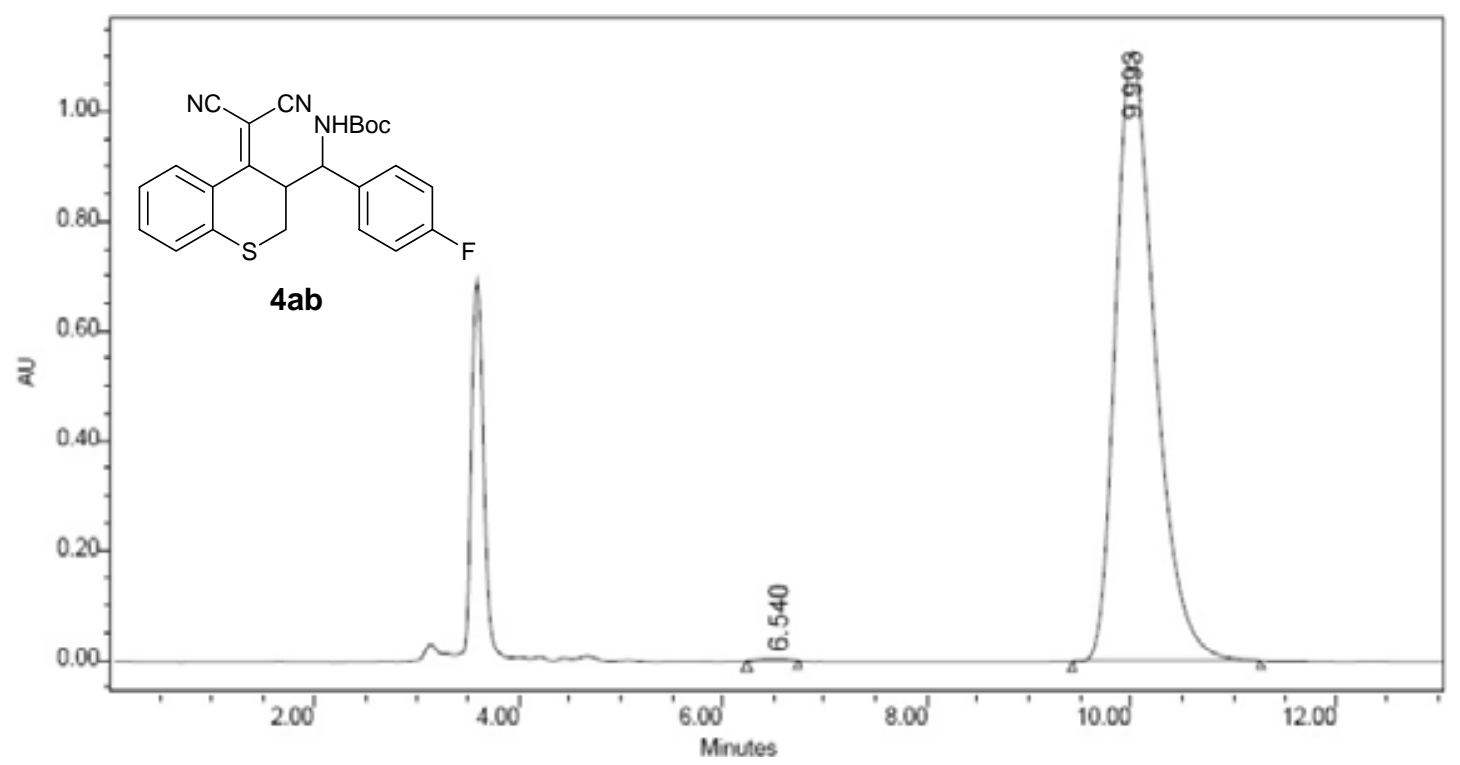

\begin{tabular}{|l|c|r|r|r|r|}
\hline & $\begin{array}{c}\text { RT } \\
(\mathrm{min})\end{array}$ & $\begin{array}{c}\text { Area } \\
\left(\mathrm{V}^{*} \mathrm{sec}\right)\end{array}$ & $\%$ Area & \multicolumn{1}{|c|}{$\begin{array}{c}\text { Height } \\
(\mathrm{V})\end{array}$} & $\begin{array}{c}\% \\
\text { Height }\end{array}$ \\
\hline 1 & 6.540 & 72514 & 0.24 & 3681 & 0.33 \\
\hline 2 & 9.993 & 29894744 & 99.76 & 1112303 & 99.67 \\
\hline
\end{tabular}

S48 


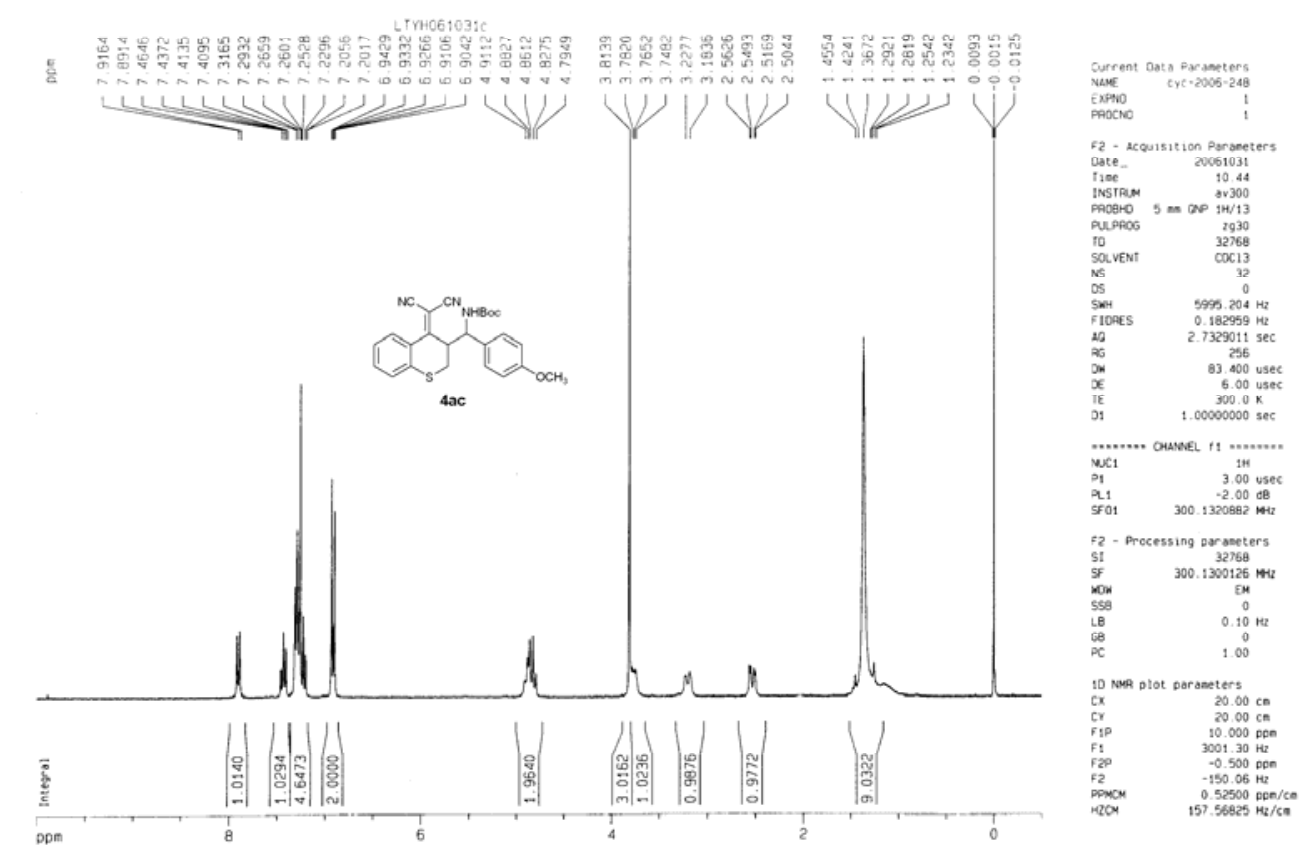

LTrM061031C

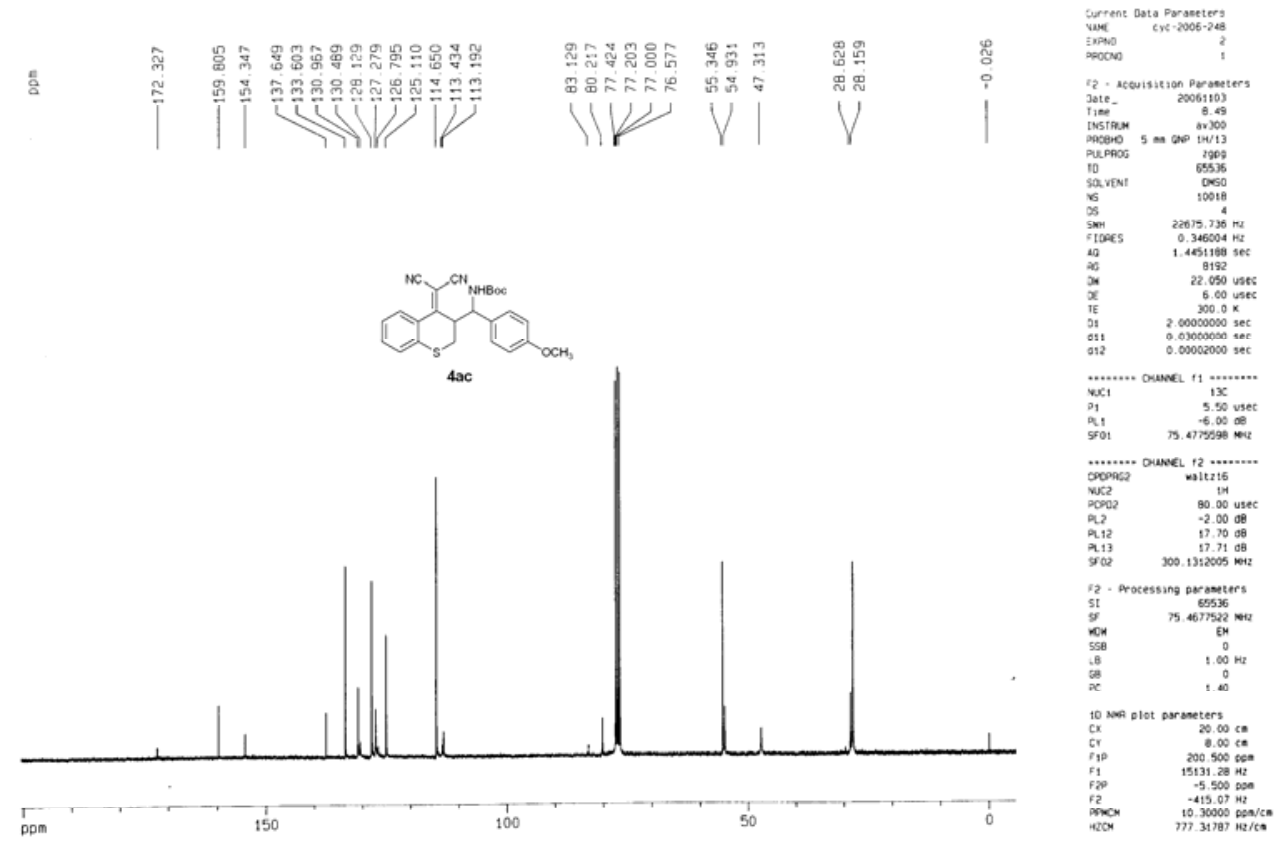




\section{Mass Spectrum Molecular Formular Report}

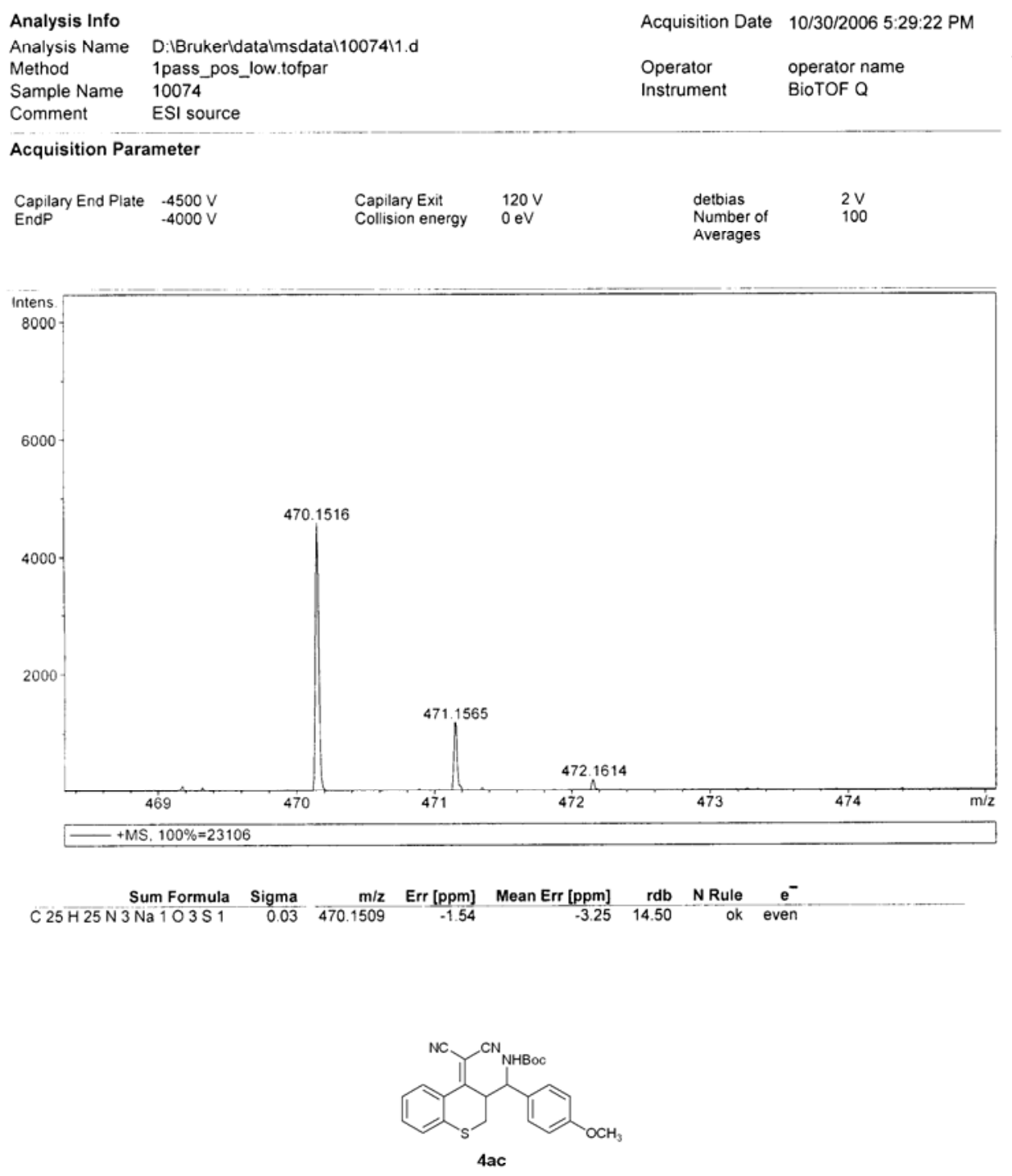




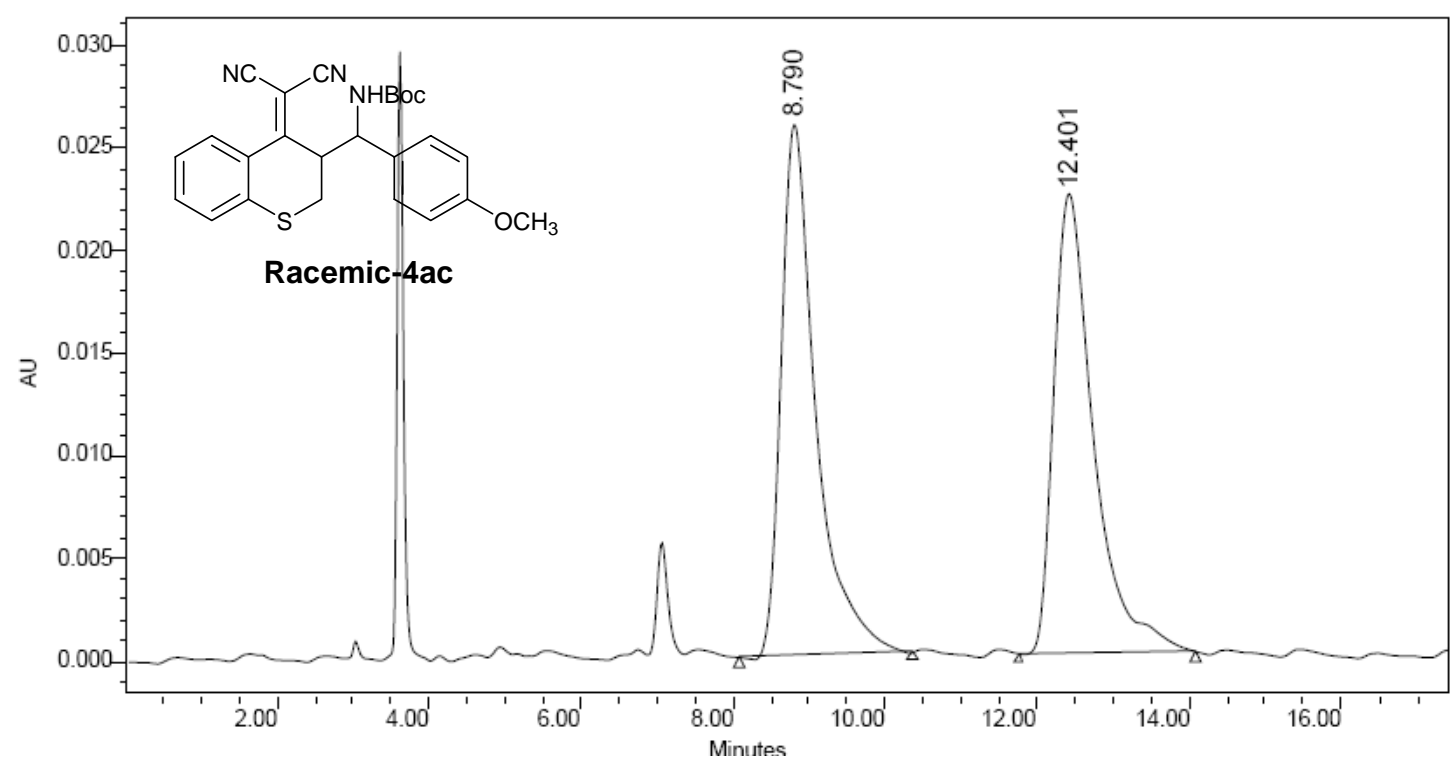

\begin{tabular}{|c|c|c|c|c|c|}
\hline & $\begin{array}{c}\text { RT } \\
(\text { min })\end{array}$ & $\begin{array}{c}\text { Area } \\
\left(V^{*} \text { sec }\right)\end{array}$ & $\%$ Area & $\begin{array}{c}\text { Height } \\
(\mathrm{V})\end{array}$ & $\begin{array}{c}\% \\
\text { Height }\end{array}$ \\
\hline 1 & 8.790 & 824604 & 50.27 & 25903 & 53.62 \\
\hline 2 & 12.401 & 815875 & 49.73 & 22402 & 46.38 \\
\hline
\end{tabular}

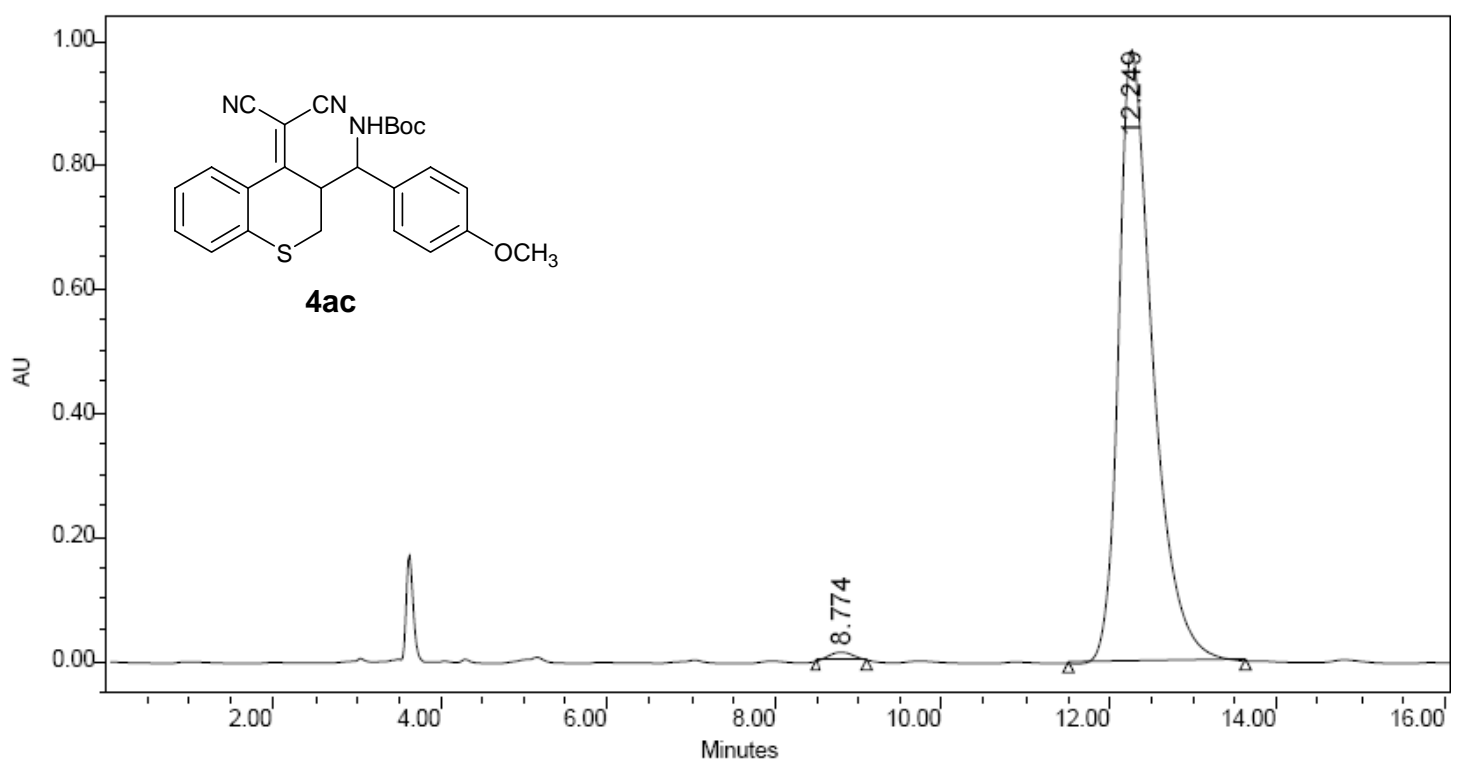

\begin{tabular}{|c|c|r|r|r|r|}
\hline & $\begin{array}{c}\mathrm{RT} \\
(\mathrm{min})\end{array}$ & $\begin{array}{c}\text { Area } \\
(\mathrm{V} \text { *sec })\end{array}$ & $\%$ Area & $\begin{array}{c}\text { Height } \\
(\mathrm{V})\end{array}$ & $\begin{array}{c}\% \\
\text { Height }\end{array}$ \\
\hline 1 & 8.774 & 128897 & 0.46 & 8764 & 0.88 \\
\hline 2 & 12.249 & 28180688 & 99.54 & 988155 & 99.12 \\
\hline
\end{tabular}



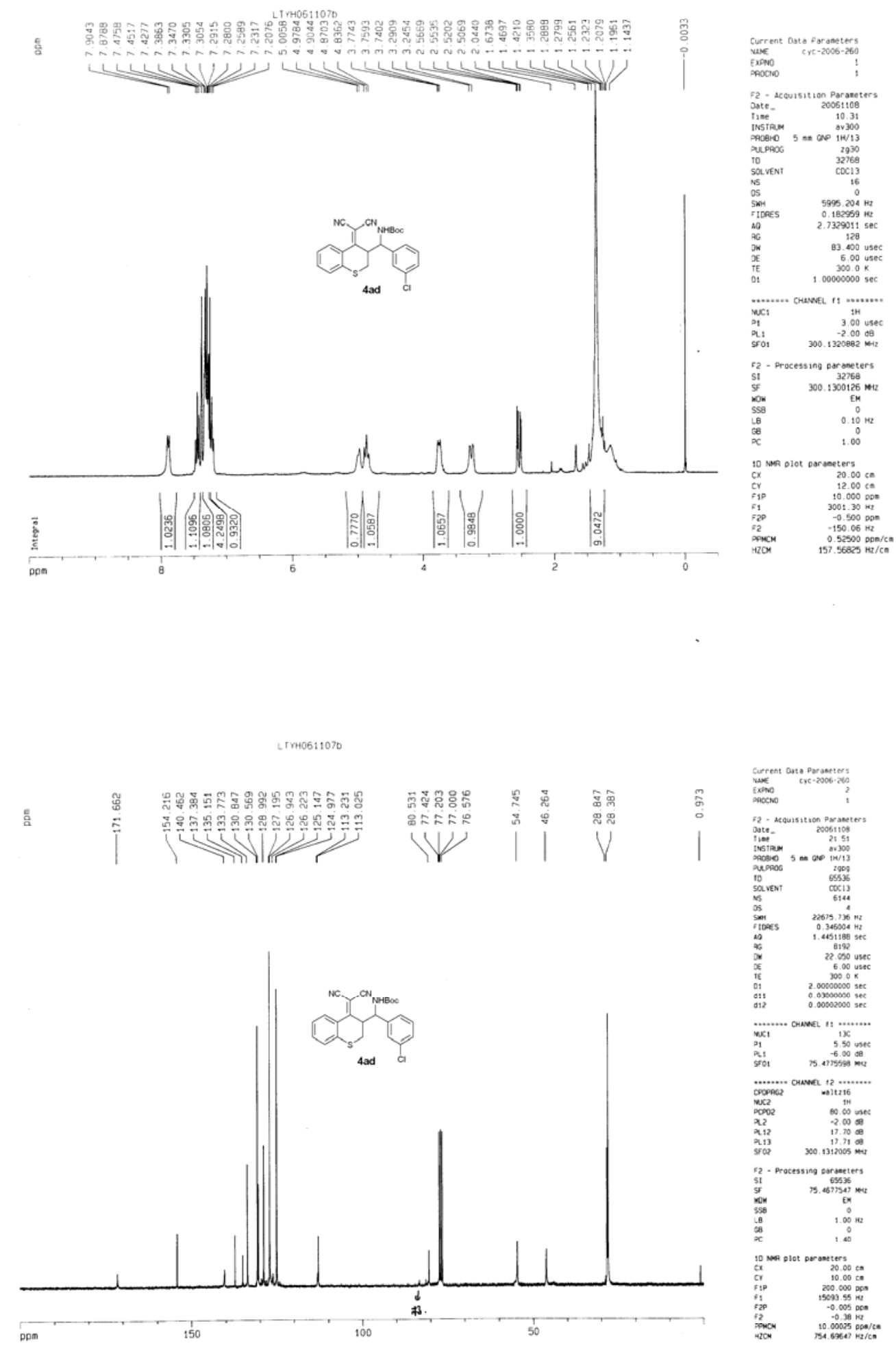


\section{Mass Spectrum Molecular Formular Report}

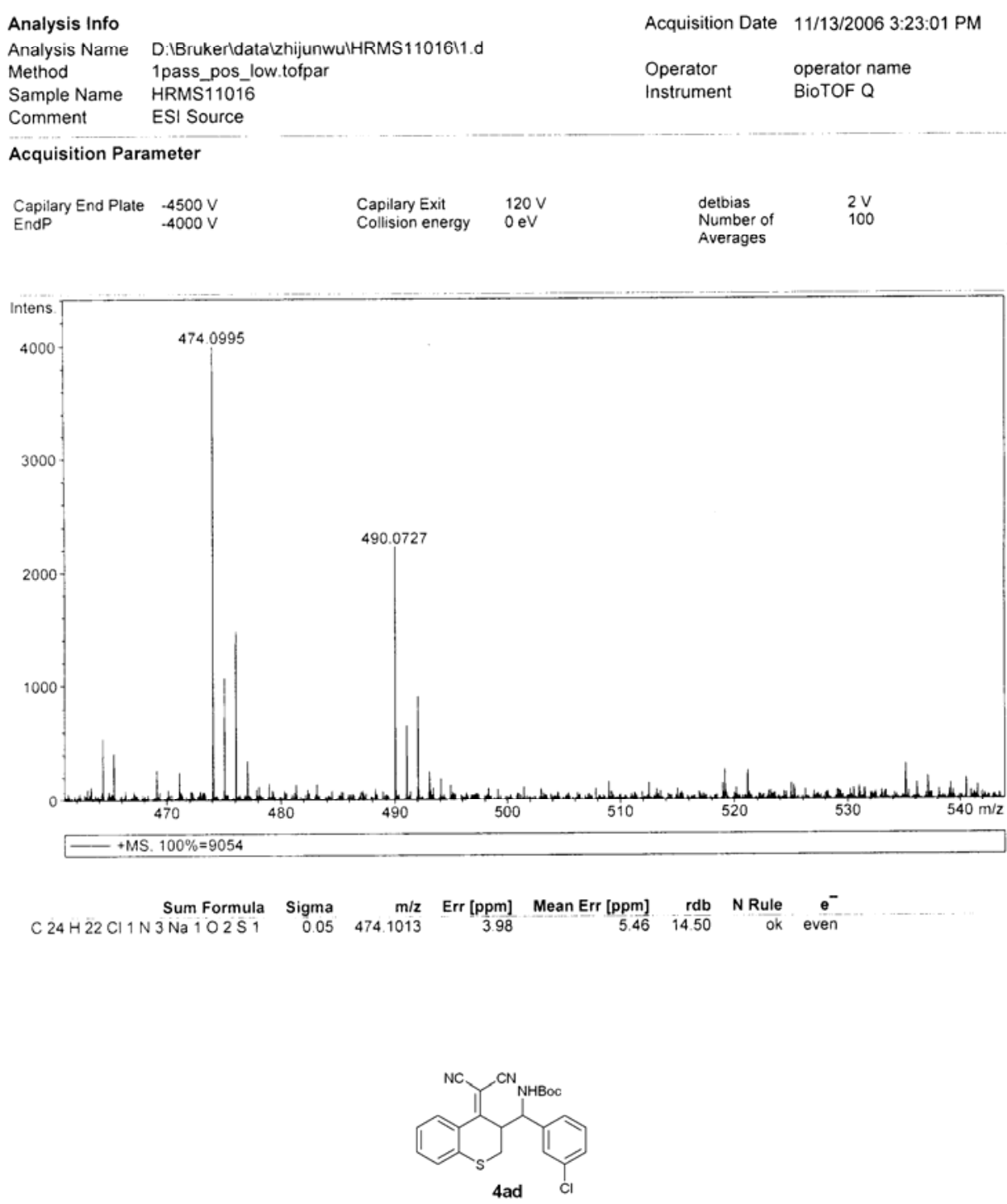



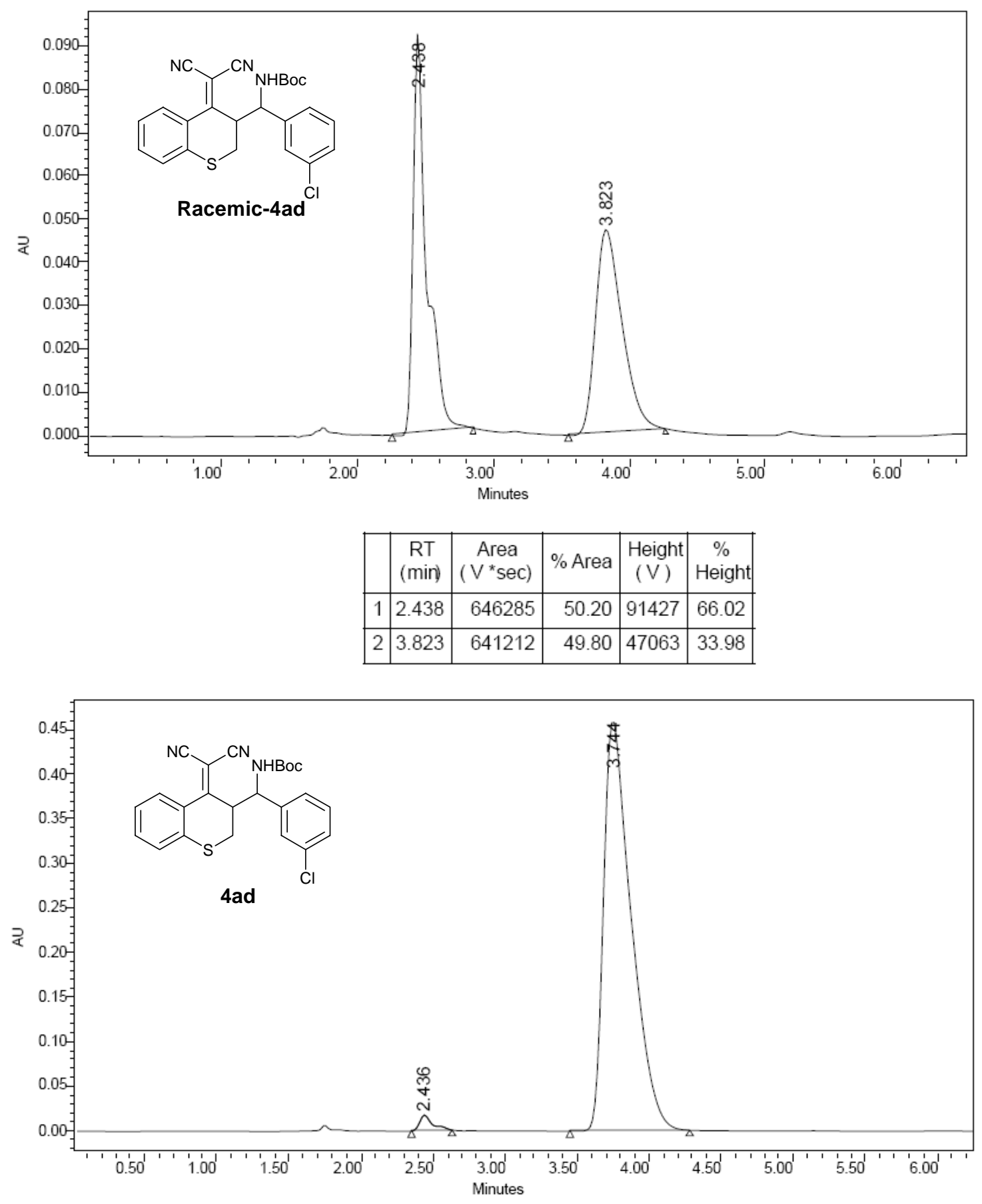

\begin{tabular}{|r|c|r|r|r|r|}
\hline & $\begin{array}{c}\mathrm{RT} \\
(\mathrm{min})\end{array}$ & $\begin{array}{c}\text { Area } \\
\left(\mathrm{V}^{*} \mathrm{sec}\right)\end{array}$ & $\%$ Area & $\begin{array}{c}\text { Height } \\
(\mathrm{V})\end{array}$ & $\begin{array}{c}\% \\
\text { Height }\end{array}$ \\
\hline 1 & 2.436 & 68192 & 1.10 & 15057 & 3.17 \\
\hline 2 & 3.744 & 6120645 & 98.90 & 459325 & 96.83 \\
\hline
\end{tabular}




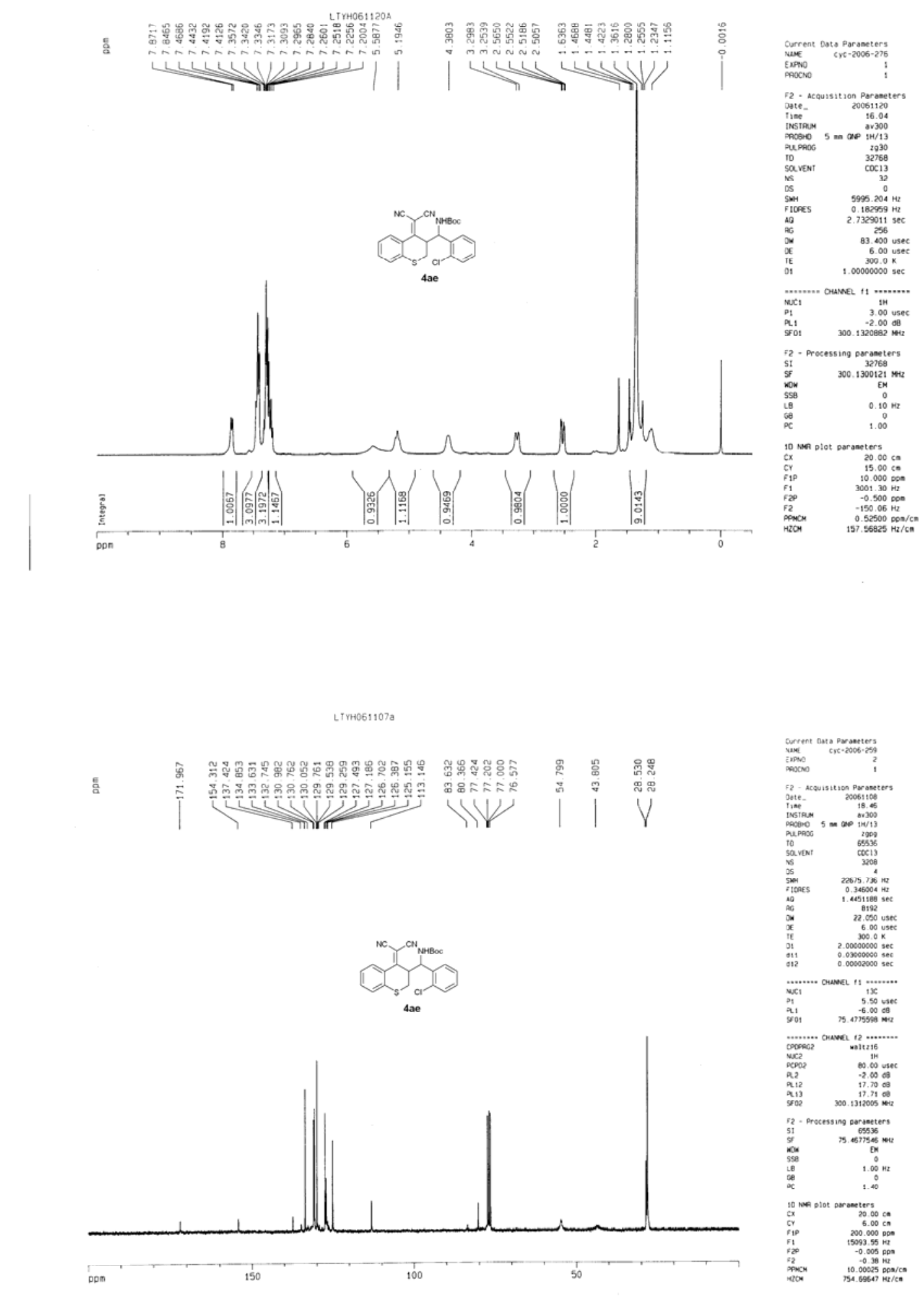




\section{Mass Spectrum Molecular Formular Report}

Analysis Info

Analysis Name D:IBrukerldatalzhijunwulHRMS11015ı1.d

Method

Sample Name

Comment

pass pos low.tofpar

HRMS11015

Acquisition Date 11/13/2006 3:31:23 PM

Operator

Instrument

operator name

BioTOF Q

Acquisition Parameter

Capilary End Plate $.4500 \mathrm{~V}$

EndP

$.4500 \mathrm{~V}$

Capilary Exit

$120 \mathrm{~V}$

detbias

Number of $\quad 100$

Averages

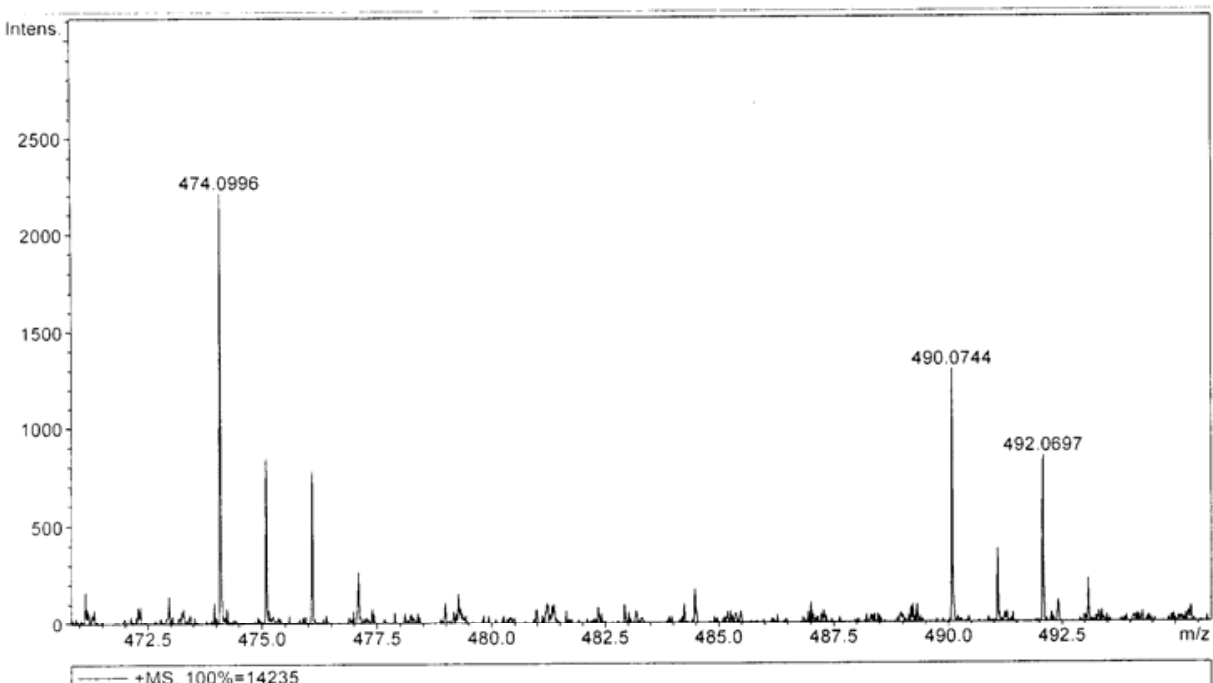

$\begin{array}{rrrrrrrr}\text { Sum Formula } & \text { Sigma } & \mathrm{m} / \mathbf{2} & \text { Err [ppm] } & \text { Mean Err [ppm] } & \text { rdb } & \text { N Rule } & \mathrm{e}^{-} \\ \mathrm{C} 24 \mathrm{H} 22 \mathrm{Cl} 1 \mathrm{~N} 3 \mathrm{Na} 102 \mathrm{~S} 1 & 0.07 & 474.1013 & 3.65 & 4.04 & 14.50 & 0 \mathrm{k} & \text { even }\end{array}$

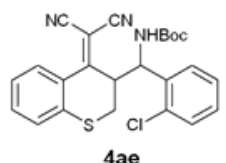

Bruker Daltonics DataAnalysis 3.2

printed: $11 / 13 / 2006 \quad \widehat{03: 33: 53} \mathrm{PM}$

Page 1 of 1 


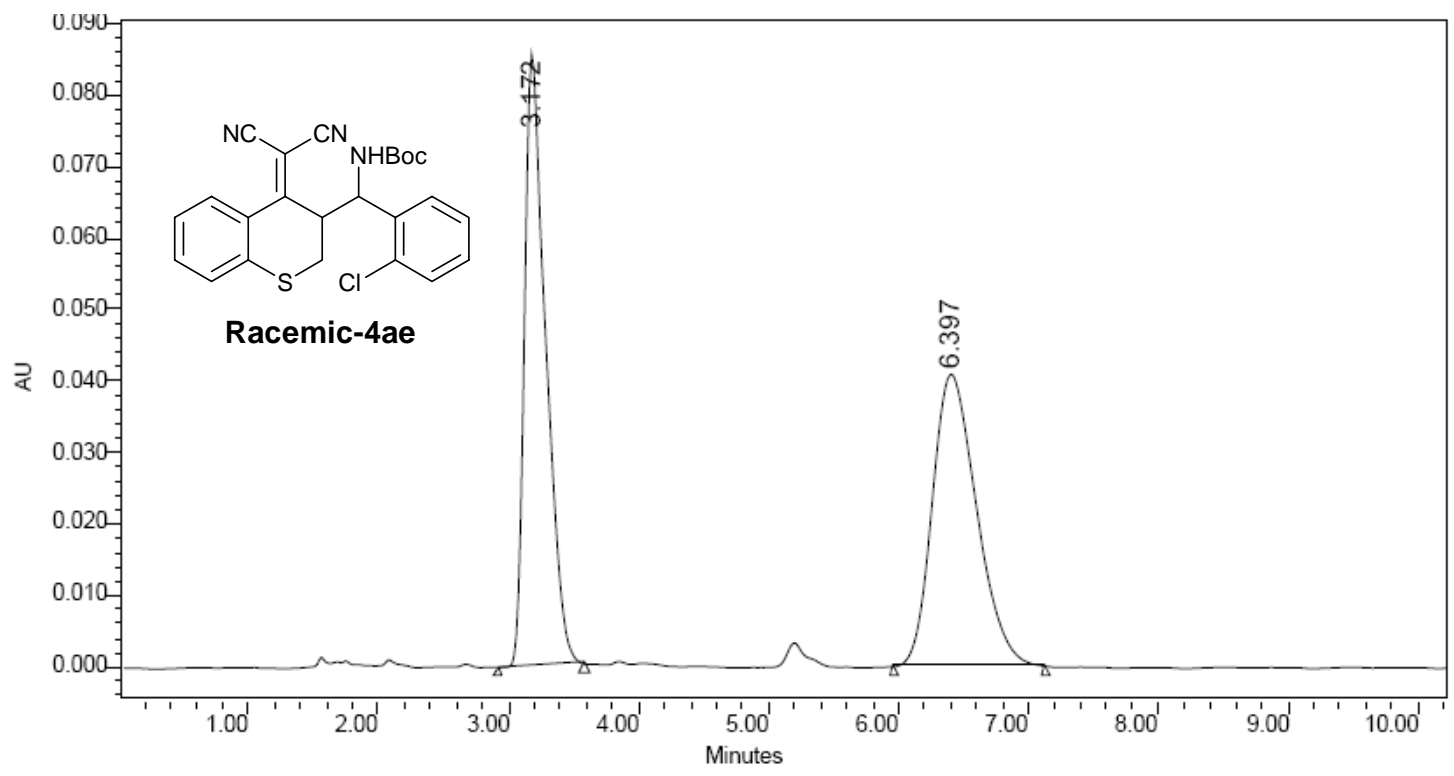

\begin{tabular}{|c|c|c|c|c|c|}
\hline & $\begin{array}{c}\mathrm{RT} \\
(\mathrm{min})\end{array}$ & $\begin{array}{c}\text { Area } \\
(\mathrm{V} \text { *sec })\end{array}$ & $\%$ Area & $\begin{array}{c}\text { Height } \\
(\mathrm{V})\end{array}$ & $\begin{array}{c}\% \\
\text { Height }\end{array}$ \\
\hline 1 & 3.172 & 973435 & 50.08 & 85729 & 67.72 \\
\hline 2 & 6.397 & 970154 & 49.92 & 40872 & 32.28 \\
\hline
\end{tabular}

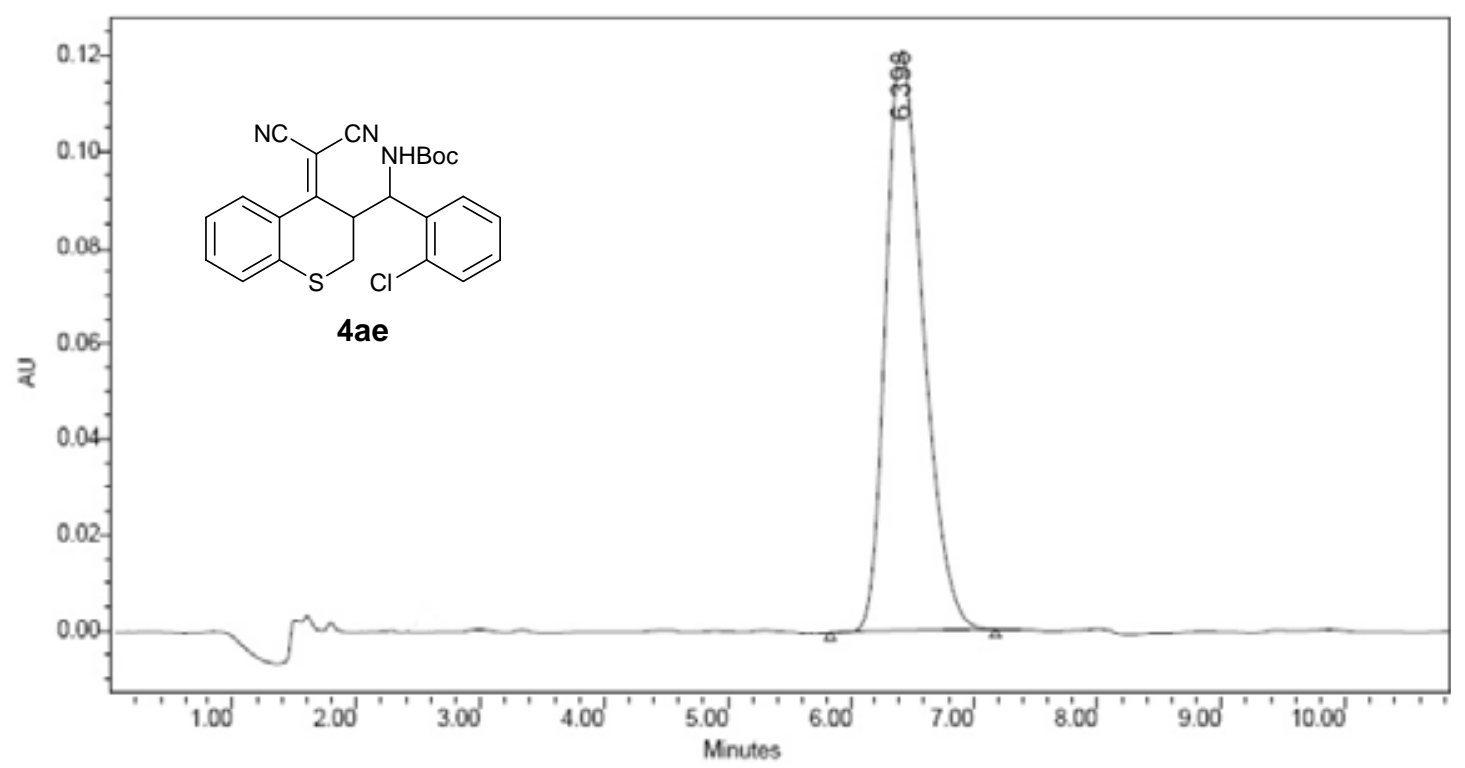

\begin{tabular}{|c|c|c|c|c|c|}
\hline & $\begin{array}{c}\text { RT } \\
(\mathrm{min})\end{array}$ & $\begin{array}{c}\text { Area } \\
\left(\mathrm{V}{ }^{*} \mathrm{sec}\right)\end{array}$ & $\%$ Area & $\begin{array}{c}\text { Height } \\
(\mathrm{V})\end{array}$ & $\begin{array}{c}\% \\
\text { Height }\end{array}$ \\
\hline 1 & 6.398 & 2575834 & 100.00 & 121155 & 100.00 \\
\hline
\end{tabular}



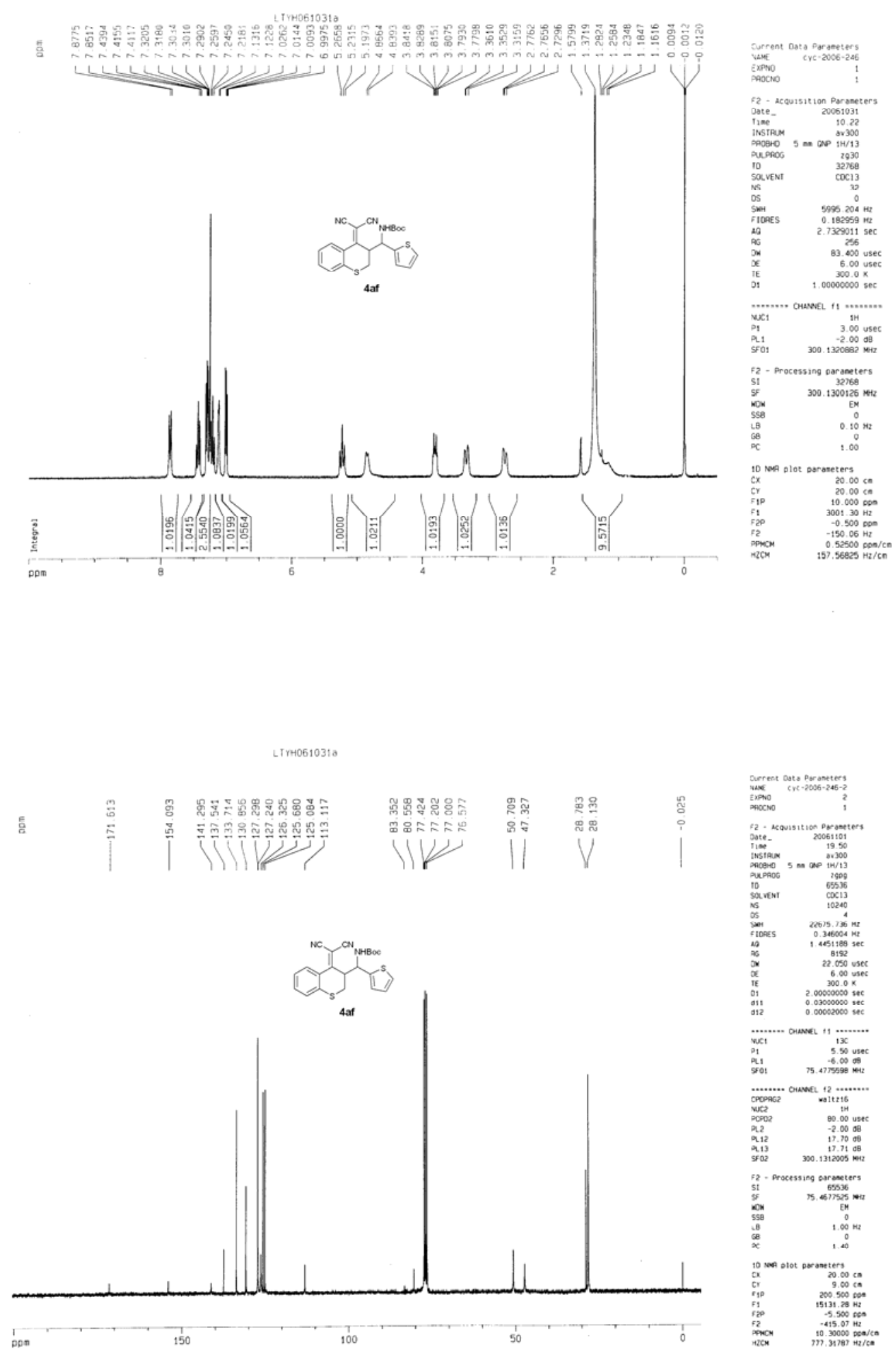
Mass Spectrum Molecular Formular Report

$\begin{array}{llll}\text { Analysis Info } & & \text { Acquisition Date } & \text { 10/30/2006 5:39:55 PM } \\ \text { Analysis Name } & \text { D:IBrukerldatalmsdatal1007812.d } & \text { Operator } & \text { operator name } \\ \text { Method } & \text { 1pass_pos_low.tofpar } & \text { Instrument } & \text { BioTOF Q } \\ \text { Sample Name } & 10078 & & \end{array}$

Comment ESI source

Acquisition Parameter

\begin{tabular}{|c|c|c|c|c|}
\hline $\begin{array}{l}\text { Capilary End Plate } \\
\text { EndP }\end{array}$ & $\begin{array}{l}.4500 \mathrm{~V} \\
.4000 \mathrm{~V}\end{array}$ & $\begin{array}{l}\text { Capilary Exit } \\
\text { Collision energy }\end{array}$ & $\begin{array}{l}120 \mathrm{~V} \\
0 \mathrm{eV}\end{array}$ & $\begin{array}{l}\text { detbias } \\
\text { Number of } \\
\text { Averages }\end{array}$ \\
\hline
\end{tabular}

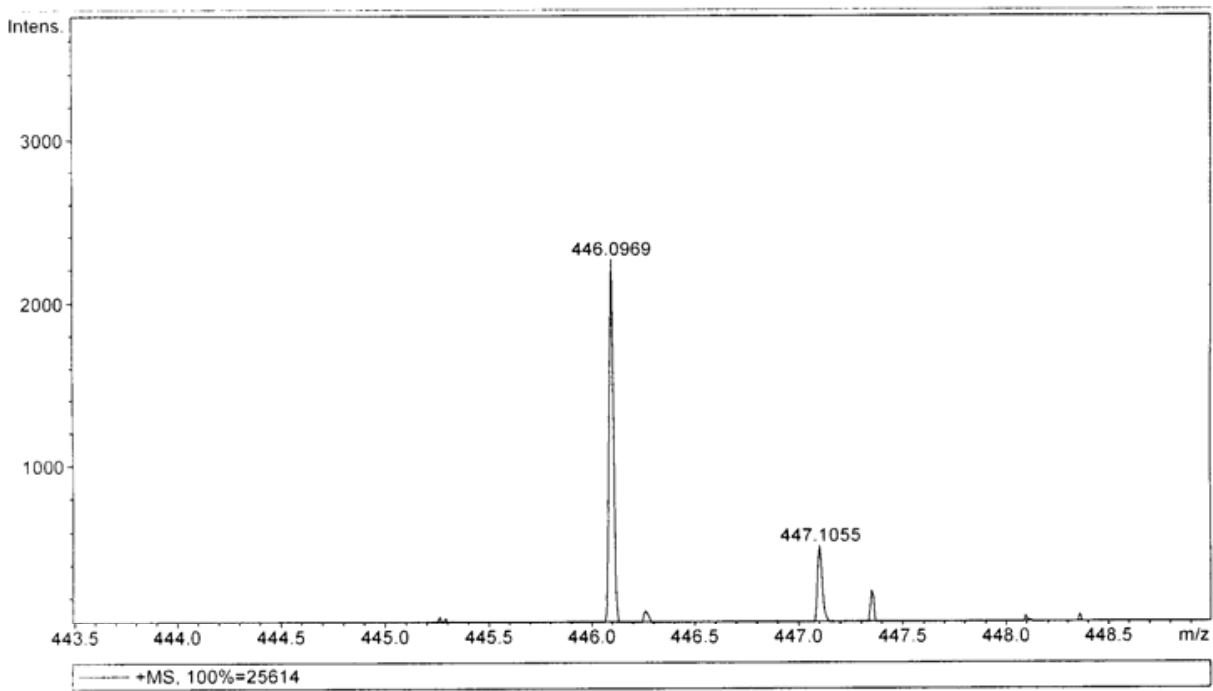

$\begin{array}{rrrrrrrr}\text { Sum Formula } & \text { Sigma } & \mathrm{m} / \mathrm{z} & \text { Err [ppm] } & \text { Mean Err [ppm] } & \text { rdb } & \text { N Rule } & e^{-} \\ \mathrm{C} 22 \mathrm{H} 21 \mathrm{~N} 3 \mathrm{Na} 102 \mathrm{~S} 2 & 0.07 & 446.0967 & -0.44 & -2.78 & 13.50 & \text { ok } & \text { even }\end{array}$

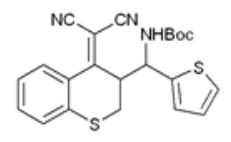

4af 


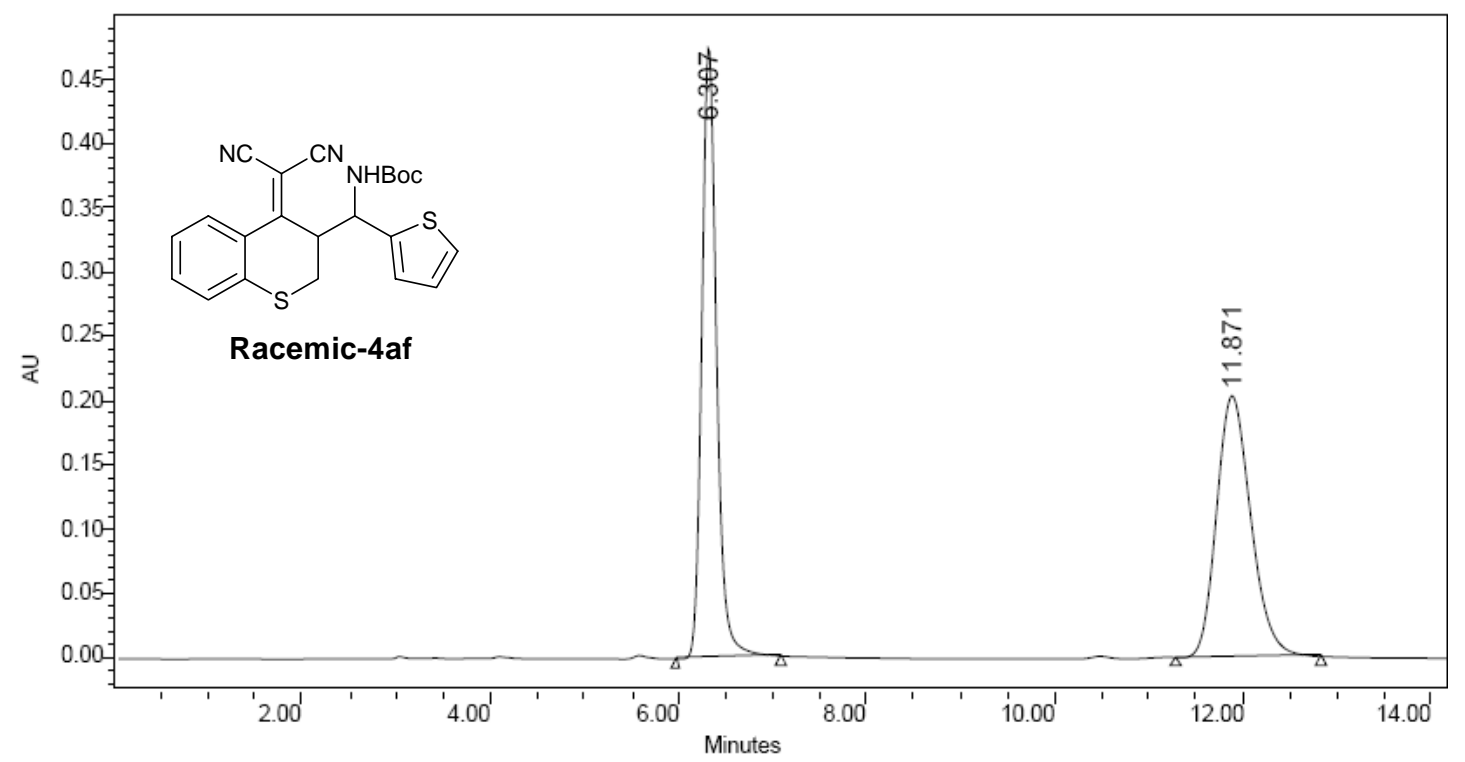

\begin{tabular}{|c|c|c|c|c|c|}
\hline & $\begin{array}{c}\mathrm{RT} \\
(\mathrm{min})\end{array}$ & $\begin{array}{c}\text { Area } \\
\left(\mathrm{V}^{*} \mathrm{sec}\right)\end{array}$ & $\%$ Area & $\begin{array}{c}\text { Height } \\
(\mathrm{V})\end{array}$ & $\begin{array}{c}\% \\
\text { Height }\end{array}$ \\
\hline 1 & 6.307 & 5199914 & 50.13 & 476472 & 70.05 \\
\hline 2 & 11.871 & 5173692 & 49.87 & 203684 & 29.95 \\
\hline
\end{tabular}

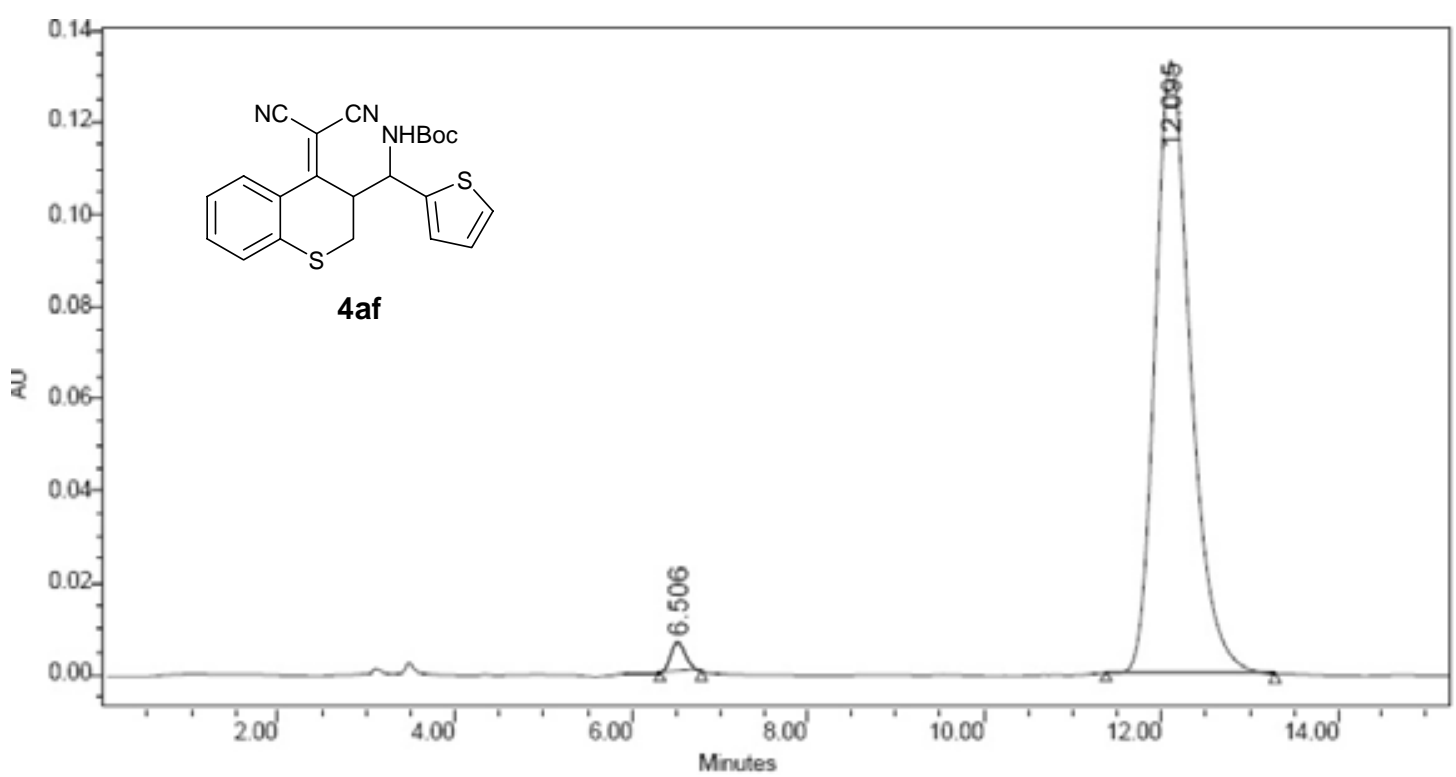

\begin{tabular}{|c|c|r|r|r|r|}
\hline & $\begin{array}{c}\text { RT } \\
(\mathrm{min})\end{array}$ & $\begin{array}{c}\text { Area } \\
\left(\mathrm{V}{ }^{*} \mathrm{sec}\right)\end{array}$ & $\%$ Area & $\begin{array}{c}\text { Height } \\
(\mathrm{V})\end{array}$ & $\begin{array}{c}\% \\
\text { Height }\end{array}$ \\
\hline 1 & 6.506 & 44569 & 1.19 & 4931 & 3.58 \\
\hline 2 & 12.095 & 3703534 & 98.81 & 132829 & 96.42 \\
\hline
\end{tabular}




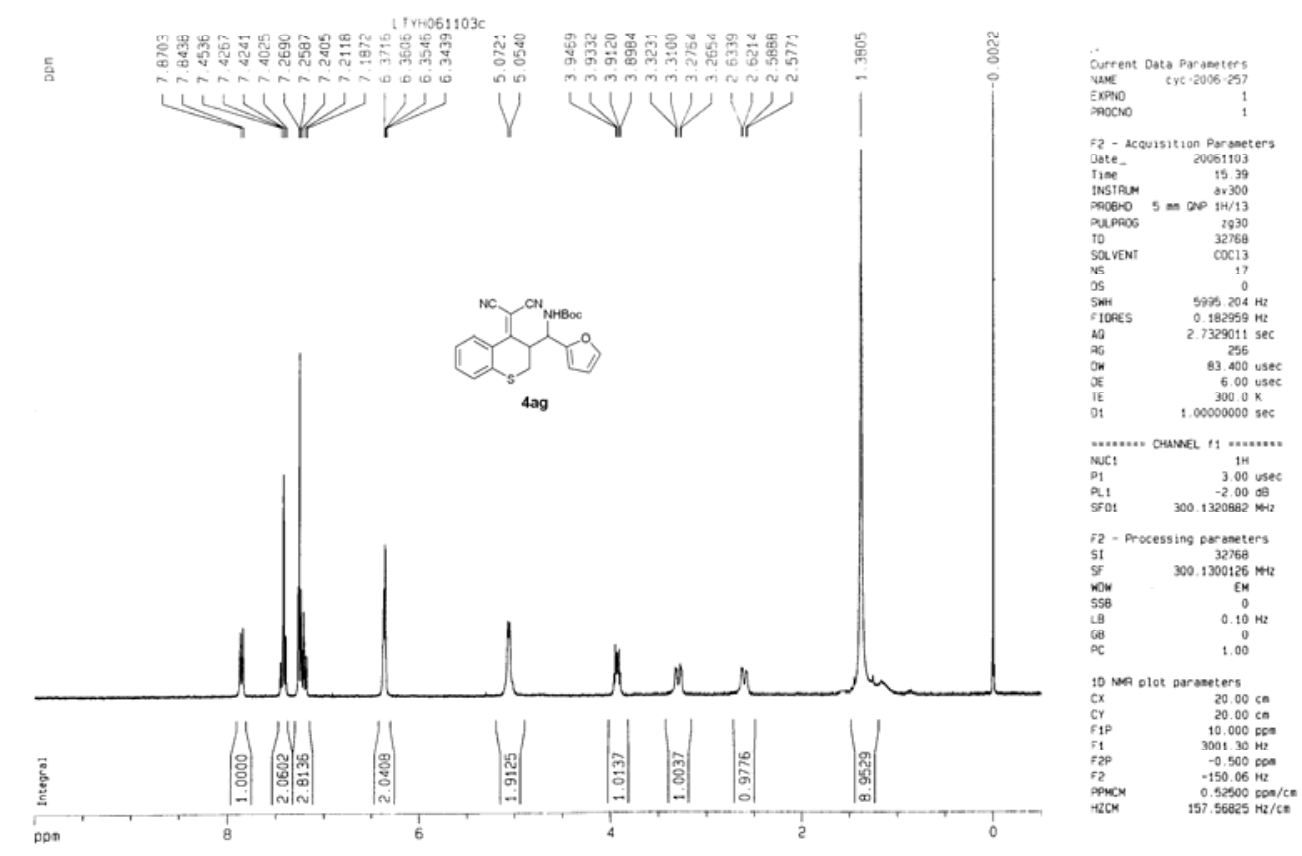

LIrHo61103C

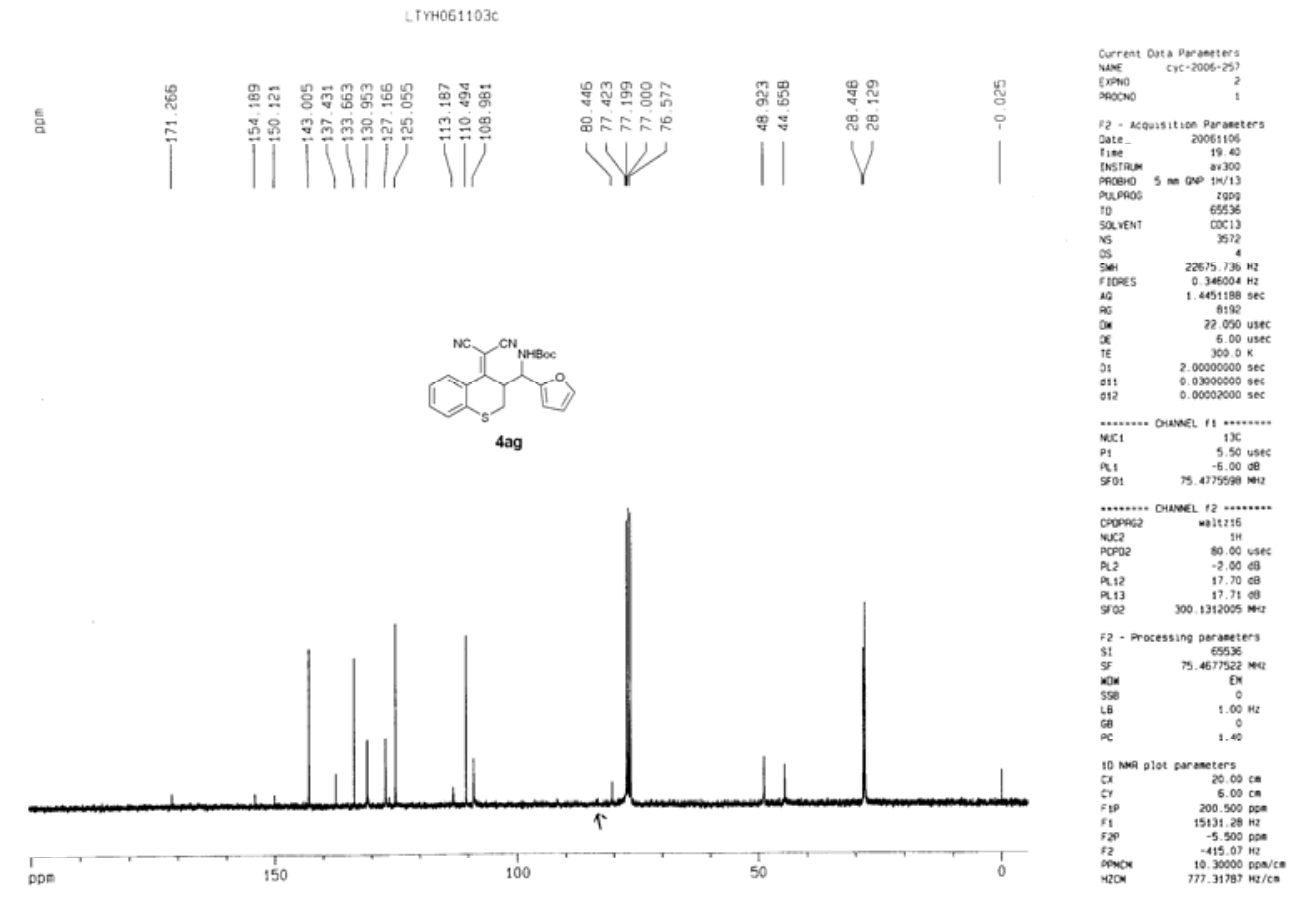




\section{Mass Spectrum Molecular Formular Report}

$\begin{array}{llll}\text { Analysis Info } & & \text { Acquisition Date } & \text { 11/13/2006 3:20:07 PM } \\ \text { Analysis Name } & \text { D:IBrukerldatalzhijunwulHRMS1010611.d } & & \\ \text { Method } & \text { 1pass_pos_low.tofpar } & \text { Operator } & \text { operator name } \\ \text { Sample Name } & \text { HRMS10106 } & \text { Instrument } & \text { BioTOF Q } \\ \text { Comment } & \text { ESI Source } & & \end{array}$

Acquisition Parameter

\begin{tabular}{|c|c|c|c|c|}
\hline $\begin{array}{l}\text { Capilary End Plate } \\
\text { EndP }\end{array}$ & $\begin{array}{l}-4500 \mathrm{~V} \\
-4000 \mathrm{~V}\end{array}$ & $\begin{array}{l}\text { Capilary Exit } \\
\text { Collision energy }\end{array}$ & $\begin{array}{l}120 \mathrm{~V} \\
0 \mathrm{eV}\end{array}$ & $\begin{array}{l}\text { detbias } \\
\text { Number of } \\
\text { Averages }\end{array}$ \\
\hline
\end{tabular}

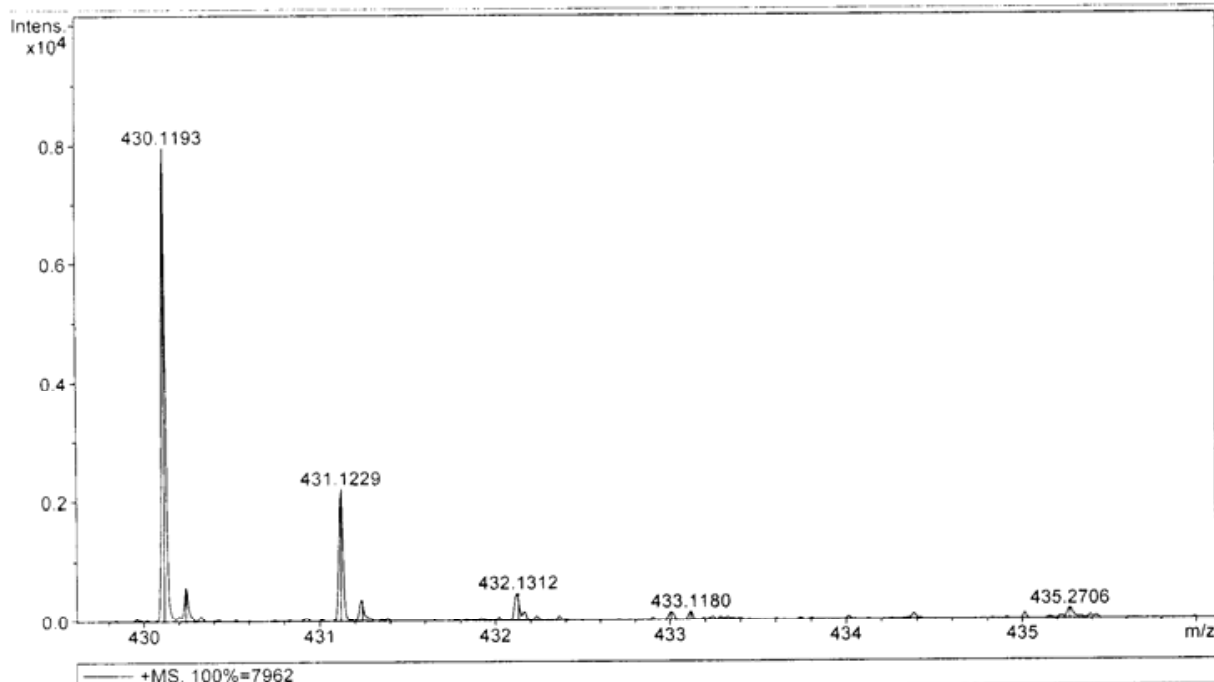

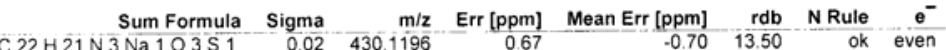

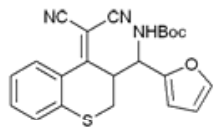

4ag 


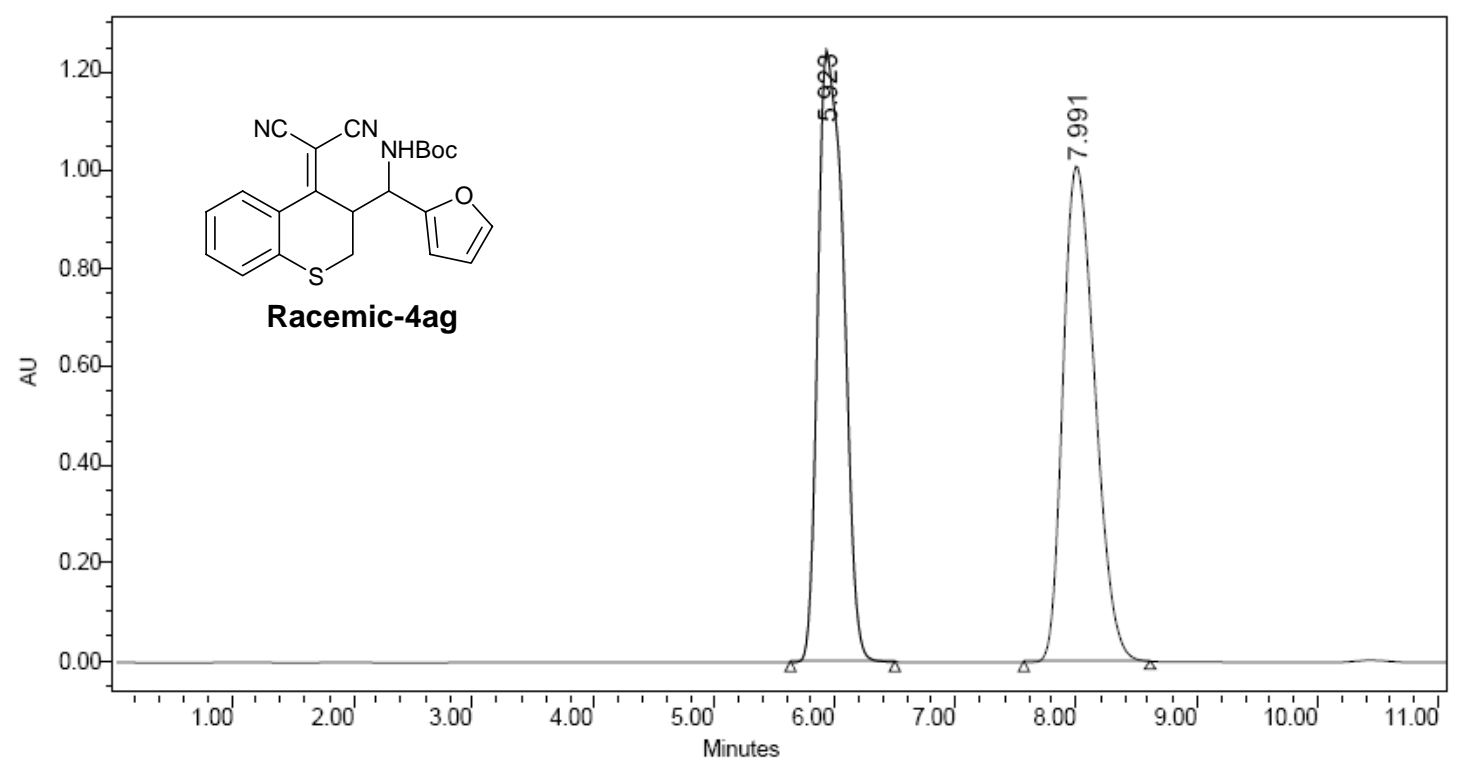

\begin{tabular}{|c|c|c|c|c|c|}
\hline & $\begin{array}{c}\mathrm{RT} \\
(\mathrm{min})\end{array}$ & $\begin{array}{c}\text { Area } \\
(\mathrm{V} \text { *sec })\end{array}$ & $\%$ Area & $\begin{array}{c}\text { Height } \\
(\mathrm{V})\end{array}$ & $\begin{array}{c}\% \\
\text { Height }\end{array}$ \\
\hline 1 & 5.923 & 18514274 & 49.98 & 1248252 & 55.24 \\
\hline 2 & 7.991 & 18526438 & 50.02 & 1011246 & 44.76 \\
\hline
\end{tabular}

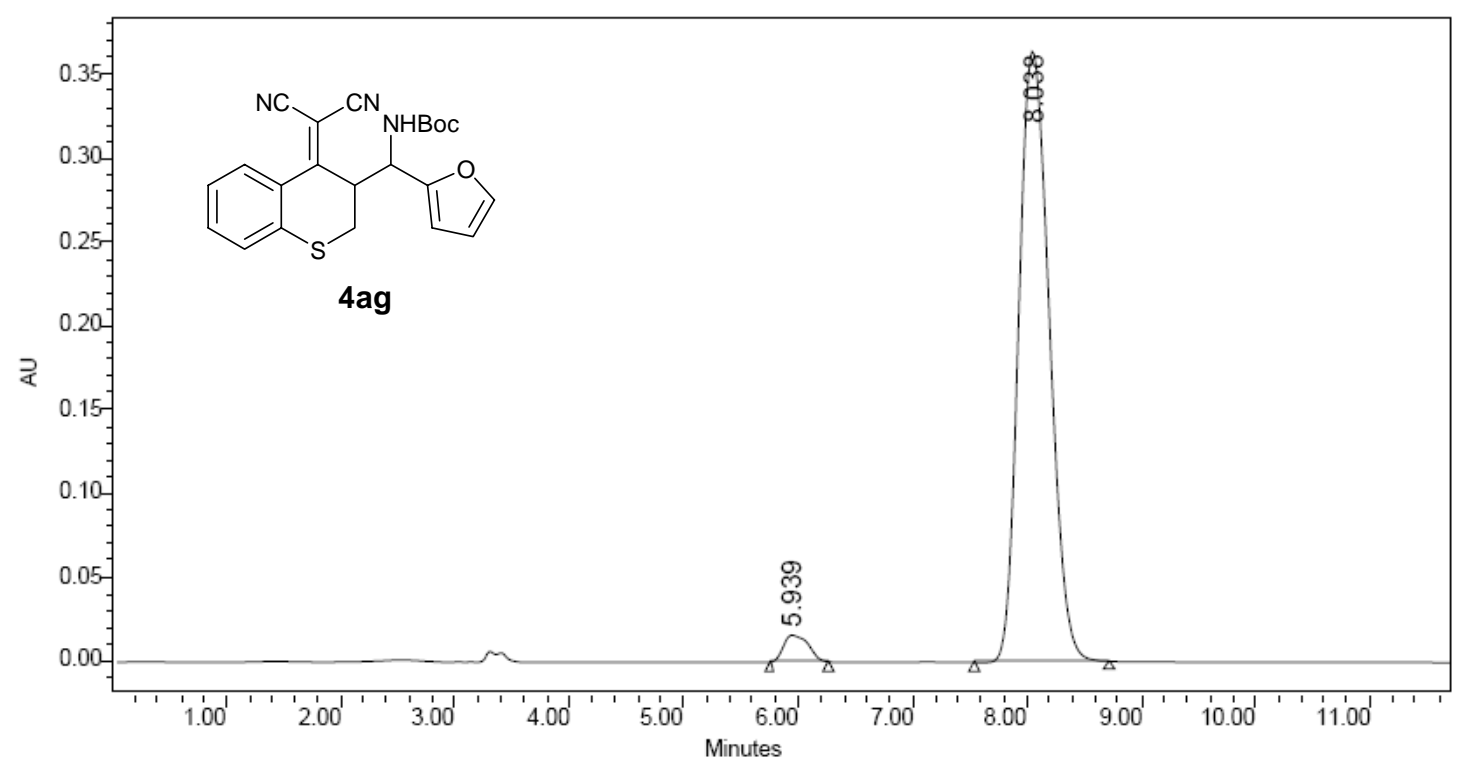

\begin{tabular}{|l|c|r|r|r|r|}
\hline & $\begin{array}{c}\text { RT } \\
(\mathrm{min})\end{array}$ & $\begin{array}{c}\text { Area } \\
\left(\mathrm{V}^{*} \mathrm{sec}\right)\end{array}$ & $\%$ Area & $\begin{array}{c}\text { Height } \\
(\mathrm{V})\end{array}$ & $\begin{array}{c}\% \\
\text { Height }\end{array}$ \\
\hline 1 & 5.939 & 146139 & 2.12 & 12110 & 3.22 \\
\hline 2 & 8.038 & 6749313 & 97.88 & 364532 & 96.78 \\
\hline
\end{tabular}



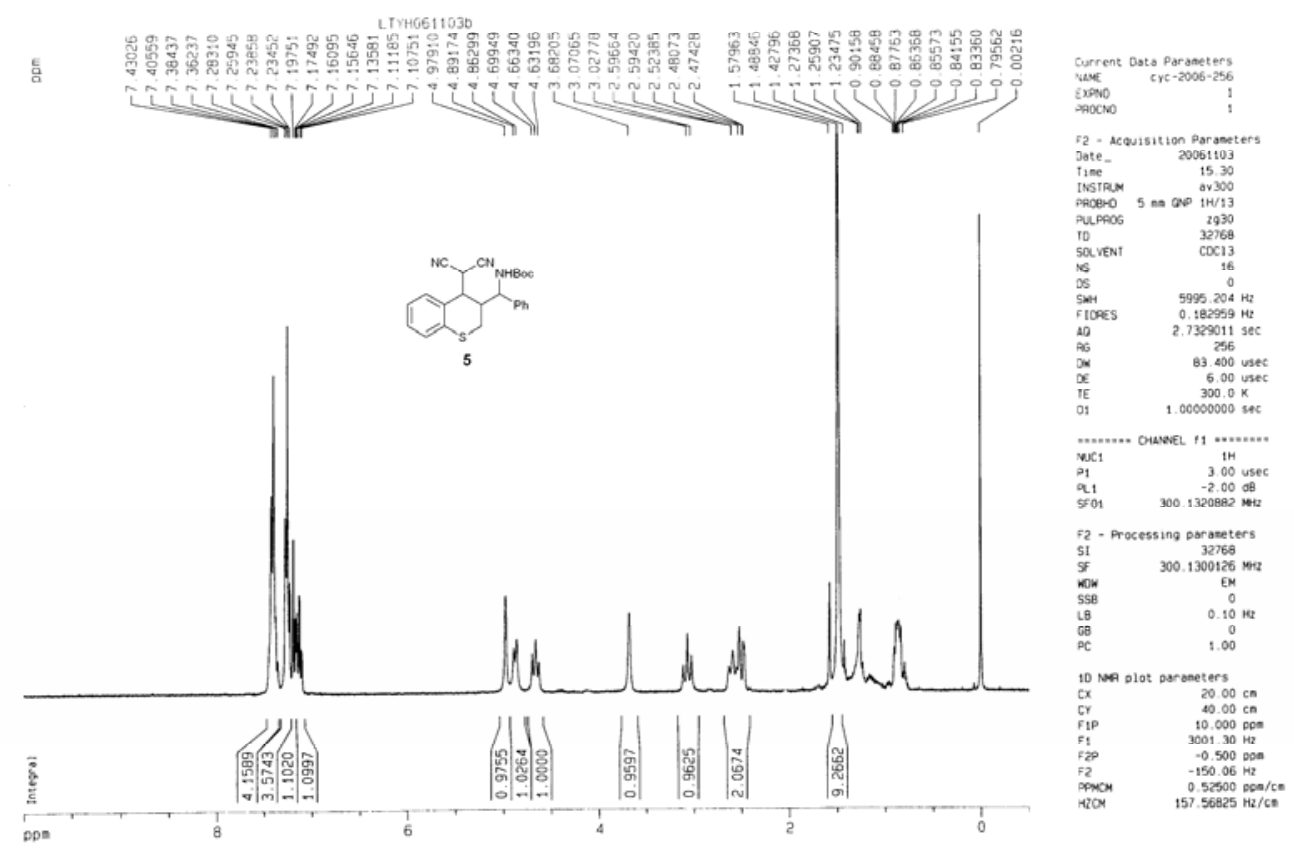

LTYH0611030

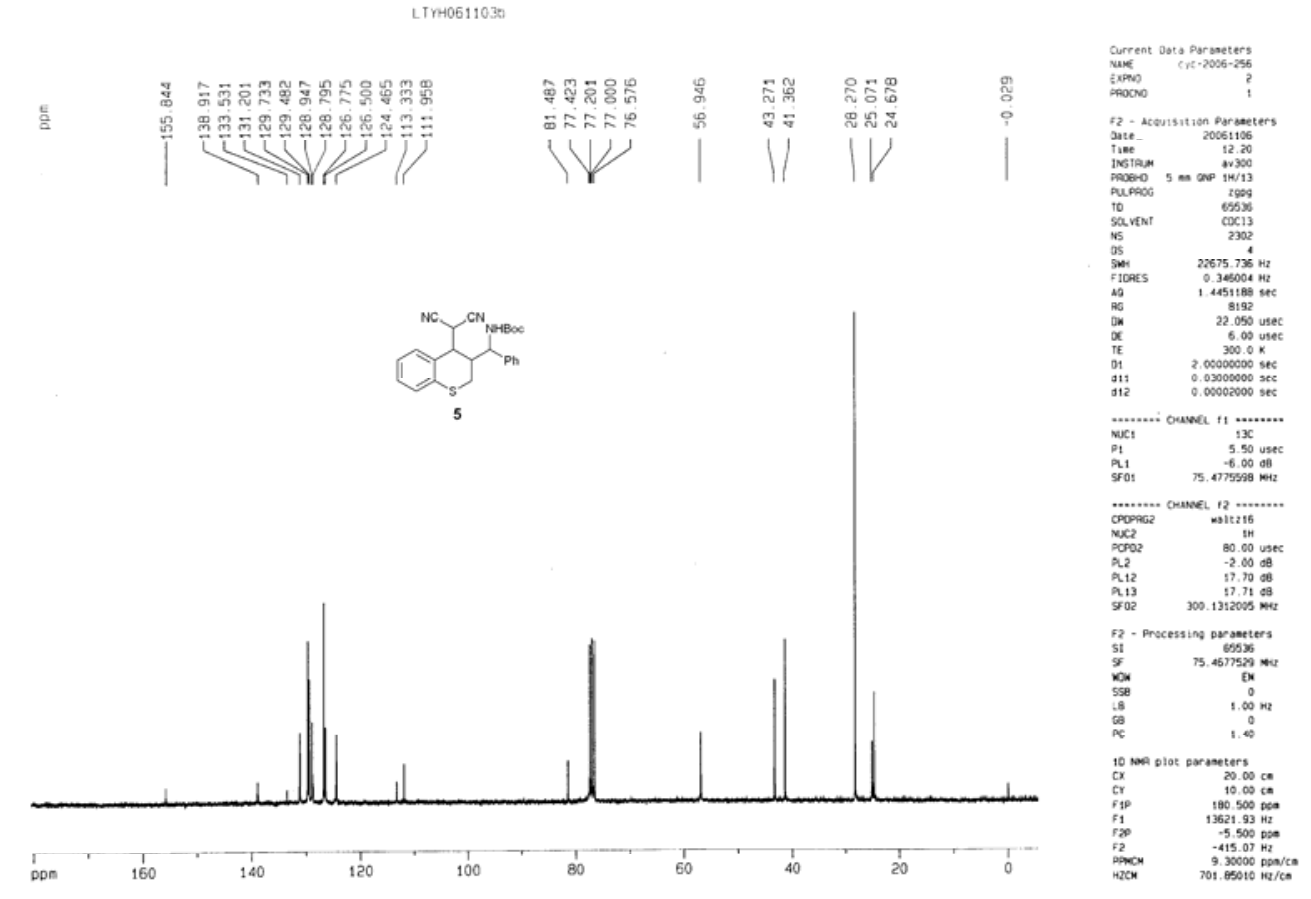


Mass Spectrum Molecular Formular Report

\begin{tabular}{|c|c|c|c|}
\hline Analysis Info & & Acquisition Date & 11/13/2006 3:16:49 PM \\
\hline Analysis Name & D:IBrukerldatalzhijunwulHRMS11012l1.d & & \\
\hline Method & 1pass_pos_low.tofpar & Operator & operator name \\
\hline Sample Name & HRMS11012 & Instrument & BioTOF Q \\
\hline Comment & ESI Source & & \\
\hline
\end{tabular}

Acquisition Parameter

\begin{tabular}{|c|c|c|c|c|}
\hline $\begin{array}{l}\text { Capilary End Plate } \\
\text { EndP }\end{array}$ & $\begin{array}{l}-4500 \mathrm{~V} \\
.4000 \mathrm{~V}\end{array}$ & $\begin{array}{l}\text { Capilary Exit } \\
\text { Collision energy }\end{array}$ & $\begin{array}{l}120 \mathrm{~V} \\
0 \mathrm{eV}\end{array}$ & $\begin{array}{l}\text { detbias } \\
\text { Number of } \\
\text { Averages }\end{array}$ \\
\hline
\end{tabular}
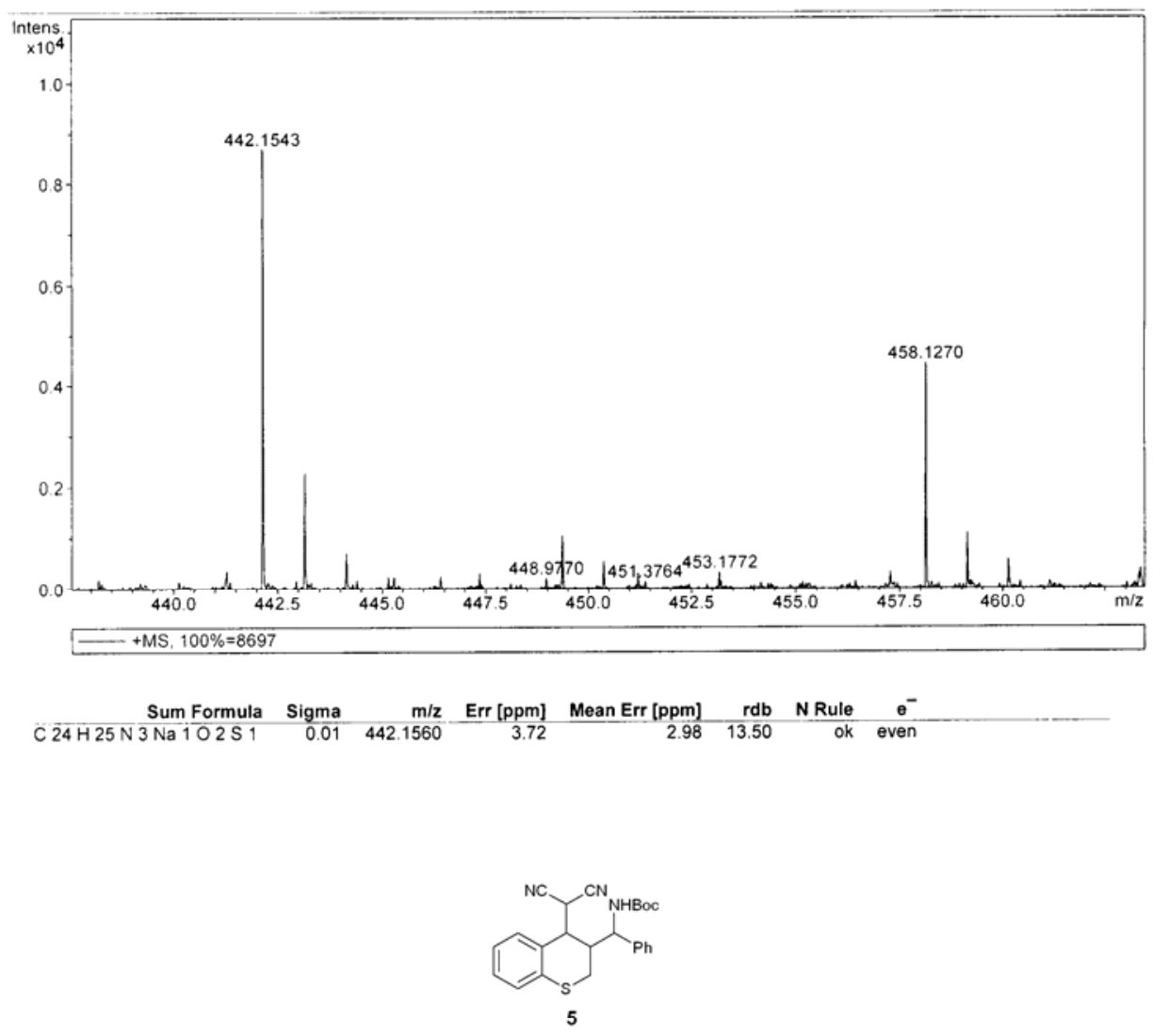

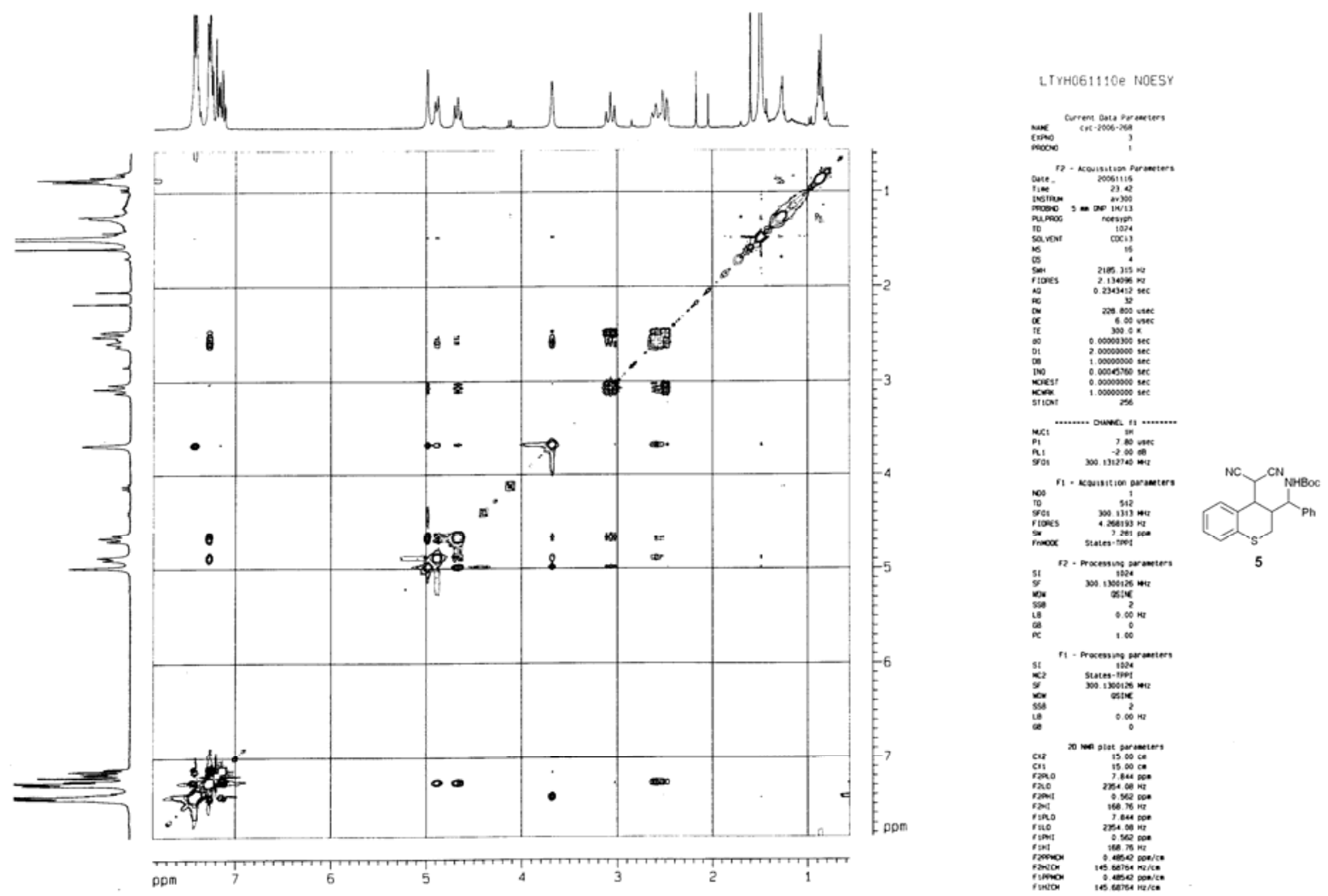


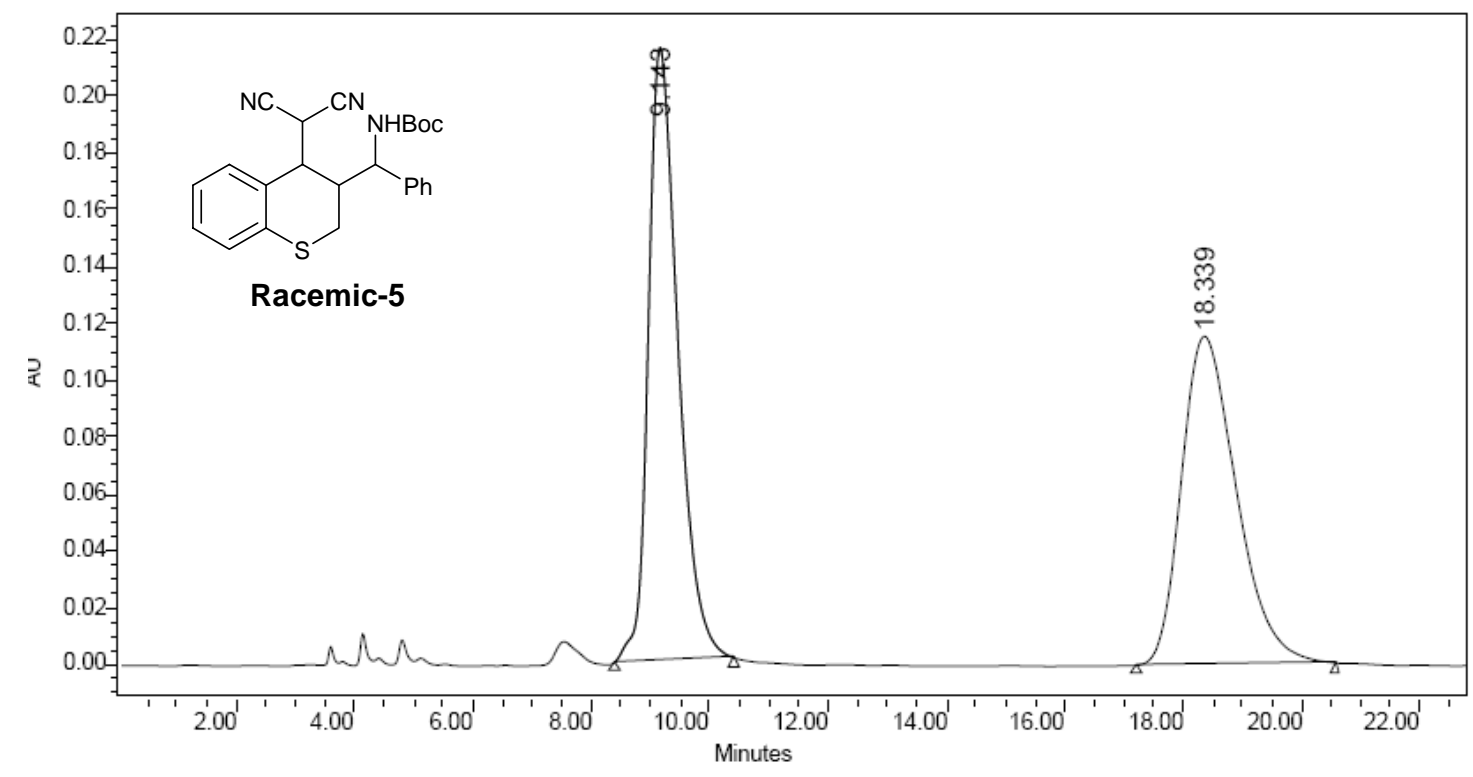

\begin{tabular}{|c|c|c|c|c|c|}
\hline & $\begin{array}{c}\mathrm{RT} \\
(\mathrm{min})\end{array}$ & $\begin{array}{c}\text { Area } \\
\left(\mathrm{V}^{*} \mathrm{sec}\right)\end{array}$ & $\%$ Area & $\begin{array}{c}\text { Height } \\
(\mathrm{V})\end{array}$ & $\begin{array}{c}\% \\
\text { Height }\end{array}$ \\
\hline 1 & 9.143 & 7535493 & 50.26 & 215766 & 65.13 \\
\hline 2 & 18.339 & 7456747 & 49.74 & 115528 & 34.87 \\
\hline
\end{tabular}

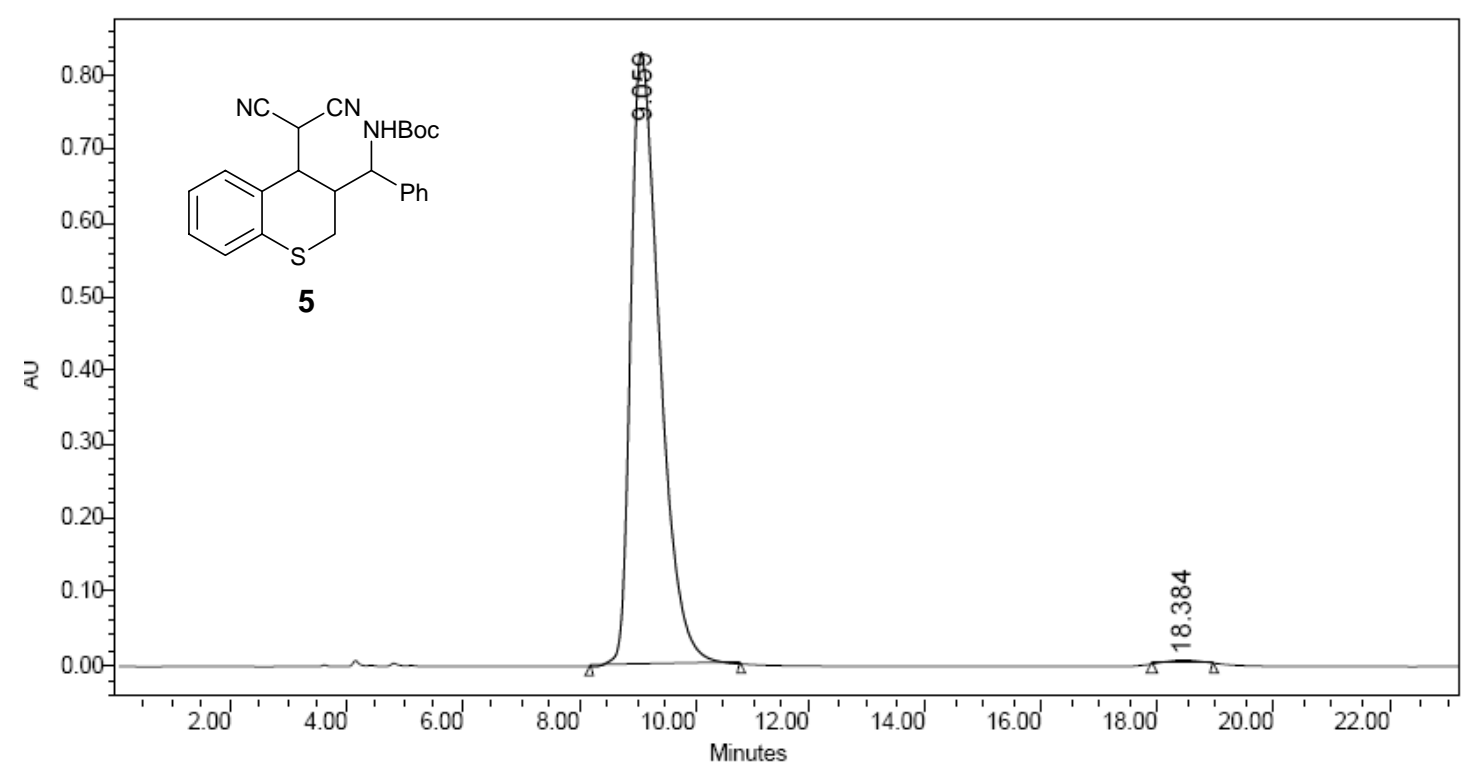

\begin{tabular}{|l|c|r|r|r|r|}
\hline & $\begin{array}{c}\mathrm{RT} \\
(\mathrm{min})\end{array}$ & $\begin{array}{c}\text { Area } \\
\left(\mathrm{V}{ }^{*} \mathrm{sec}\right)\end{array}$ & $\%$ Area & $\begin{array}{c}\text { Height } \\
(\mathrm{V})\end{array}$ & $\begin{array}{c}\% \\
\text { Height }\end{array}$ \\
\hline 1 & 9.059 & 29042081 & 99.38 & 832195 & 99.46 \\
\hline 2 & 18.384 & 179848 & 0.62 & 4498 & 0.54 \\
\hline
\end{tabular}



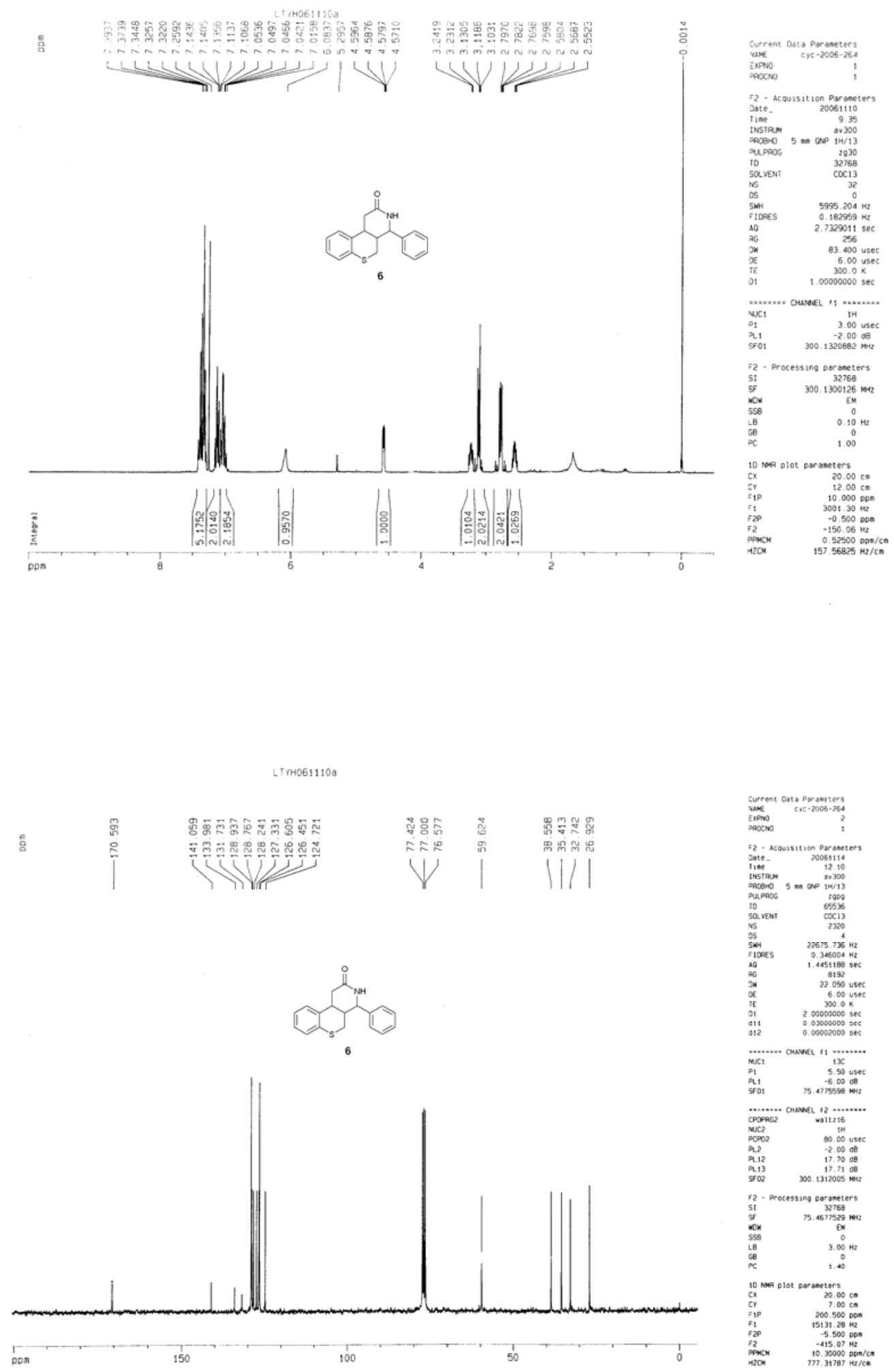


\section{Mass Spectrum Molecular Formular Report}

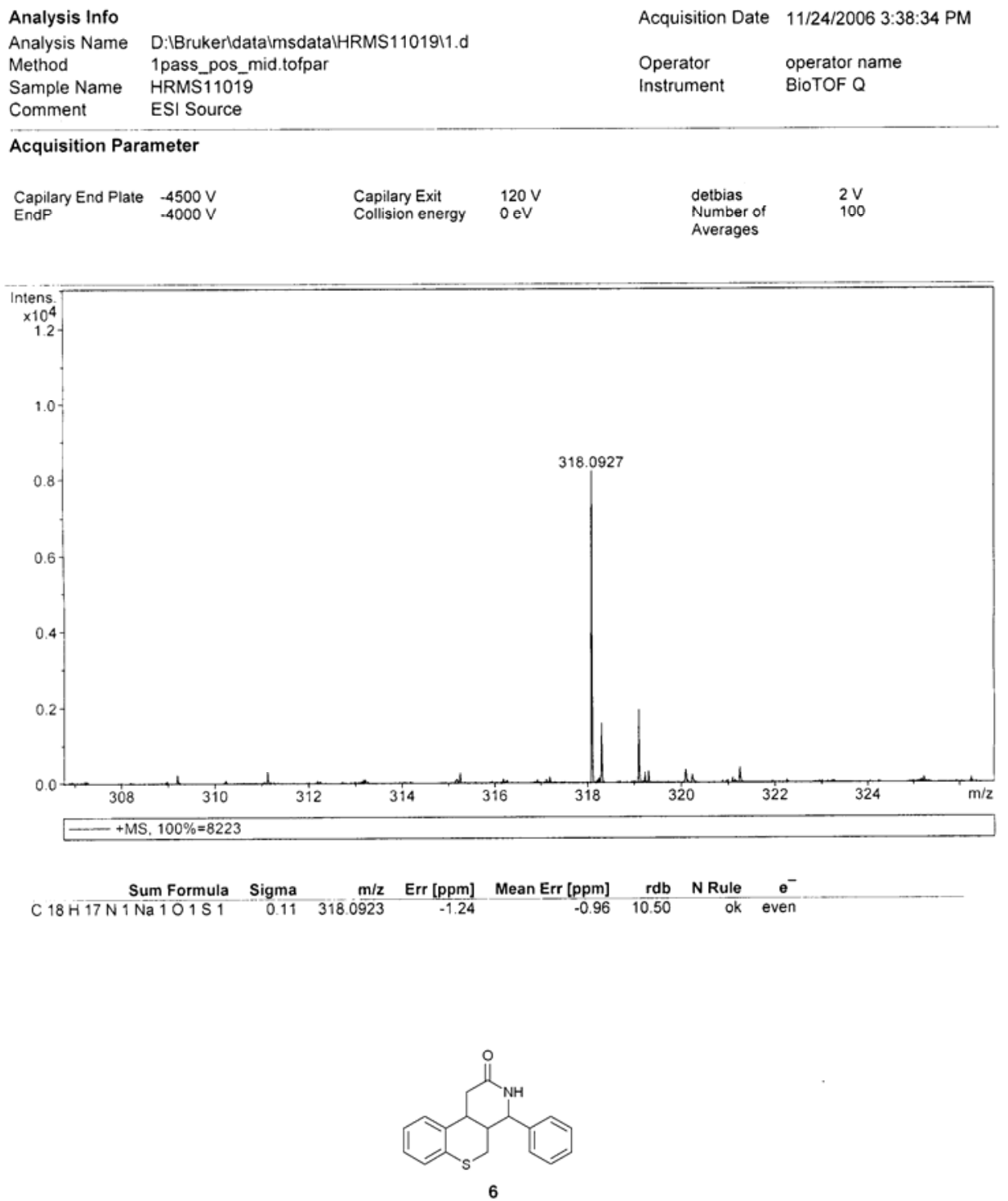




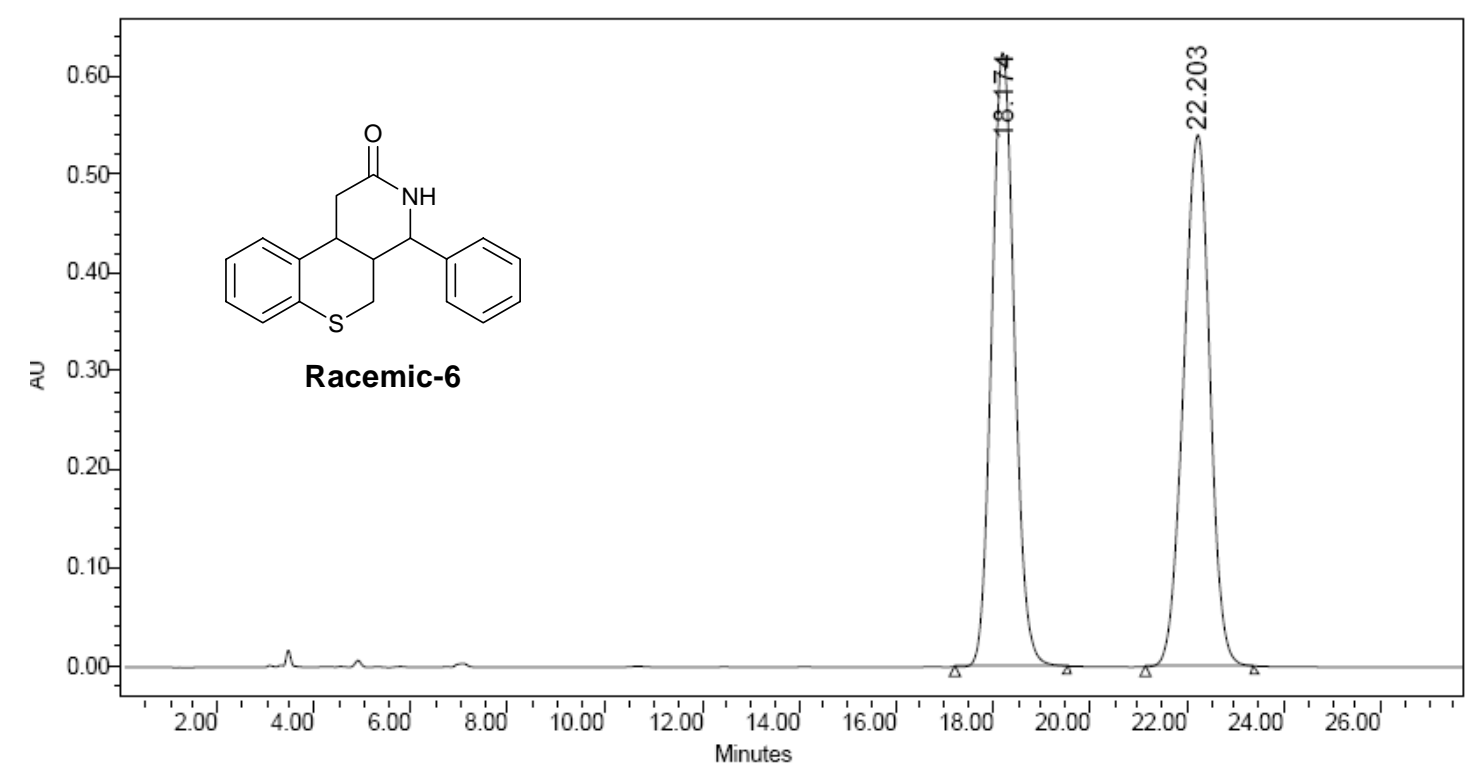

\begin{tabular}{|c|c|c|c|c|c|}
\hline & $\begin{array}{c}\text { RT } \\
(\mathrm{min})\end{array}$ & $\begin{array}{c}\text { Area } \\
\left(\mathrm{V}{ }^{*} \mathrm{sec}\right)\end{array}$ & $\%$ Area & $\begin{array}{c}\text { Height } \\
(\mathrm{V})\end{array}$ & $\begin{array}{c}\% \\
\text { Height }\end{array}$ \\
\hline 1 & 18.174 & 20375082 & 49.99 & 624727 & 53.60 \\
\hline 2 & 22.203 & 20384086 & 50.01 & 540869 & 46.40 \\
\hline
\end{tabular}

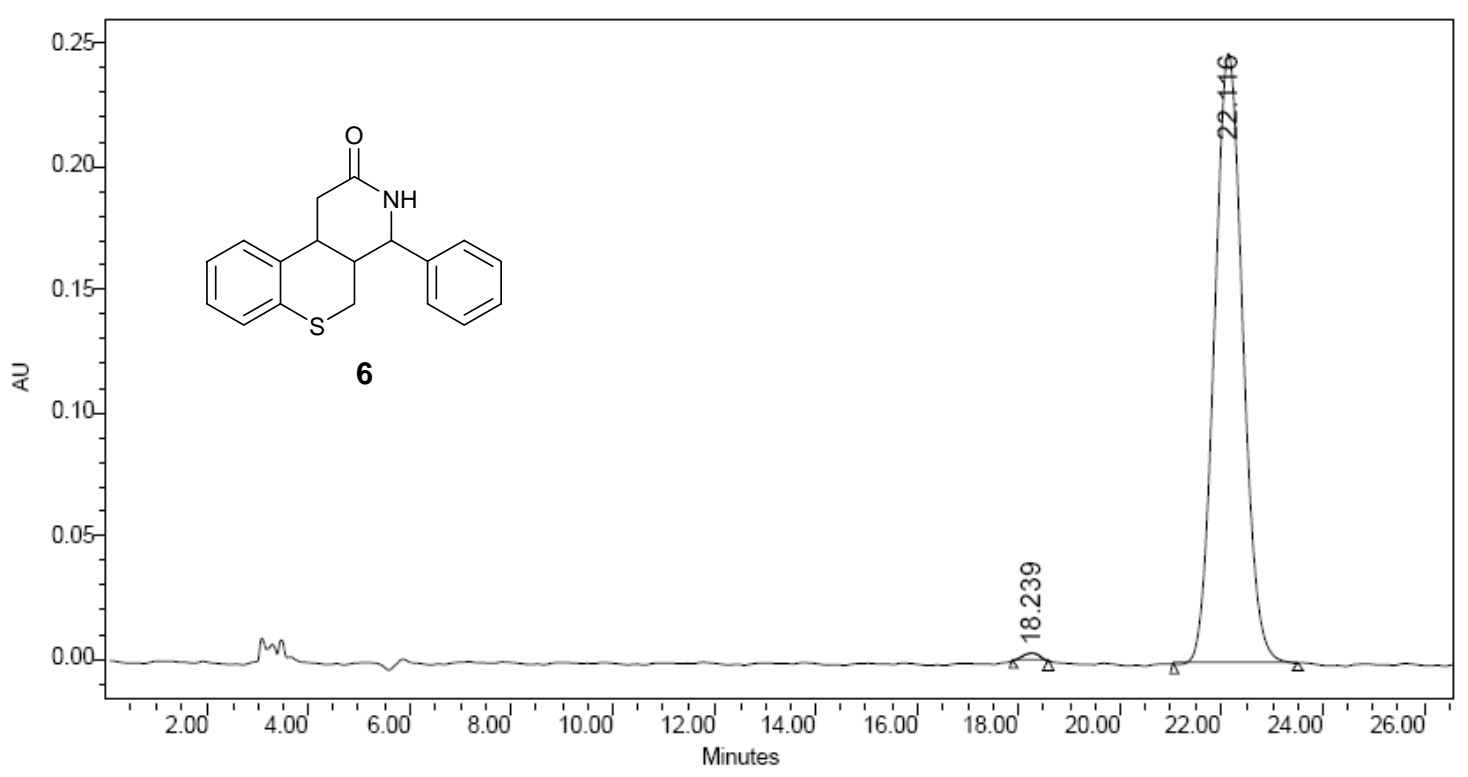

\begin{tabular}{|c|c|r|r|r|r|}
\hline & $\begin{array}{c}\mathrm{RT} \\
(\mathrm{min})\end{array}$ & $\begin{array}{c}\text { Area } \\
\left(\mathrm{V}{ }^{*} \mathrm{sec}\right)\end{array}$ & $\%$ Area & $\begin{array}{c}\text { Height } \\
(\mathrm{V})\end{array}$ & $\begin{array}{c}\% \\
\text { Height }\end{array}$ \\
\hline 1 & 18.239 & 72068 & 0.74 & 3122 & 1.24 \\
\hline 2 & 22.116 & 9653567 & 99.26 & 247642 & 98.76 \\
\hline
\end{tabular}

